\title{
A Numerical Study of Deposition in a Full Turbine Stage Using Steady and Unsteady Methods
}

\section{THESIS}

Presented in Partial Fulfillment of the Requirements for the Degree Master of Science in the Graduate School of The Ohio State University

By

Daniel Anthony Zagnoli, B.S.

Graduate Program in Aeronautical and Astronautical Engineering

The Ohio State University

2015

Master's Examination Committee:

Dr. Jeffrey Bons, Advisor

Dr. Ali Ameri

Dr. Jen-Ping Chen 


\section{Copyright by}

Daniel A Zagnoli

2015 


\begin{abstract}
A computational study was performed to investigate deposition phenomena in a highpressure turbine stage. Steady mixing-plane and unsteady sliding mesh calculations were utilized. Three-dimensional, steady and unsteady RANS calculations were performed in conjunction with published experiments completed on a similar turbine geometry which provided boundary conditions and pressure data to validate flow solutions. Particles were introduced into the flow domain and deposition was predicted using a Lagrangian particle tracking method with the critical viscosity model to predict deposition. For the steady method, in order to track particles from the mixing plane through the blade domain, particle positions were saved after passing through the vane domain and inserted into the blade domain using two different methods which were named averaged and preserved. Both methods yielded nearly identical results. For the unsteady simulation particles were tracked through a sliding mesh interface with particle position, velocity, and temperature preserved at exit of the vane domain and inlet of the blade domain. Deposition results for the steady mixing plane using both particle averaging techniques and unsteady sliding interface were compared for particles of different sizes. Large particles produce localized impact and deposit zones near the hub and tip for all methods. Steady methods deviated from unsteady methods at all particle diameters by neglecting unsteady vane wake motion causing different impact locations and subsequent multiple rebounds. At low
\end{abstract}


Stokes numbers $(2.8-11)$ the steady methods overpredicted impacts, by $30 \%$ and $25 \%$ respectively, because wake motion and particle drag dominated particle trajectories, pulling them away from pressure surface. At a high Stokes number (31) the steady method underpredicted impacts and deposits as wake motion caused a shift in initial impact locations. However, the larger particle inertia of these particles allowed subsequent impacts on adjacent suction surfaces causing a large increase in impact and capture efficiencies. 


\section{Dedication}

This document is dedicated to my family, but especially to my mother, whose strength, courage, and love are a daily inspiration. 


\section{Acknowledgments}

I would like to acknowledge the support of my family, who has set a wonderful foundation and example for my life. I acknowledge my girlfriend, Amanda, who has been a source of inspiration and grounding throughout my graduate experience. I would also like acknowledge Dr. Bons and Dr. Ameri whose guidance, experience, and encouragement have been absolutely instrumental in my work. Lastly, I acknowledge my colleague and co-worker Robin Prenter for all of his tireless patience, help, and friendship. 


\section{Vita}

June 2013 B.S. Mechanical Engineering, Cornell University

August 2013 to present Graduate Research Associate, Department of Mechanical and Aerospace Engineering, The Ohio State University

\section{Publications}

Zagnoli, D., Prenter, R., and Bons, J.P., 2015, “Experimental and Numerical Study of Deposition in Pin Fin Arrays with Impingement Cooling Jets.” AIAA ASM Scitech 2015.

Zagnoli, D., Prenter, R., Ameri, A., and Bons, J.P., 2015, "Numerical Study of Deposition in a Full Turbine Stage Using Steady and Unsteady Methods.” ASME GT2015-43613.

\section{Fields of Study}

Major Field: Aeronautical and Astronautical Engineering

Specialization: Experimental and Computational Fluid Dynamics and Heat Transfer, Turbomachinery 


\section{Table of Contents}

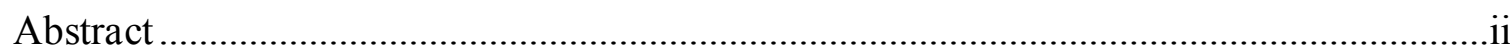

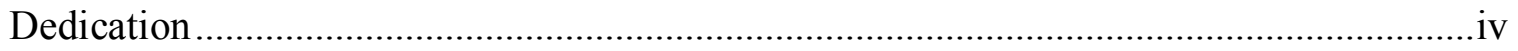

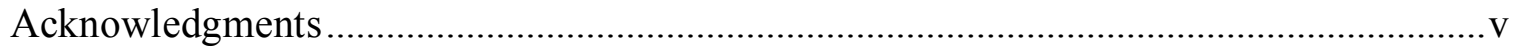

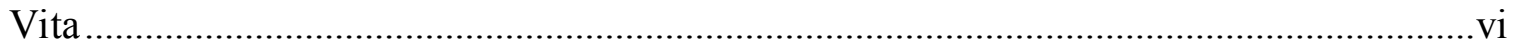

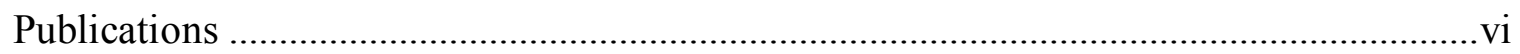

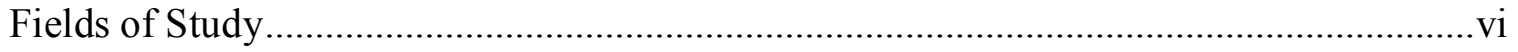

Table of Contents ............................................................................................................

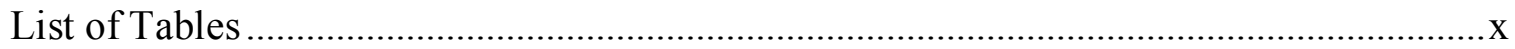

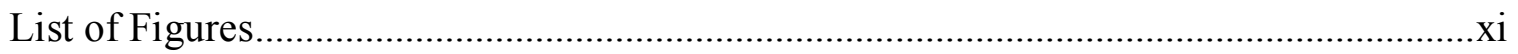

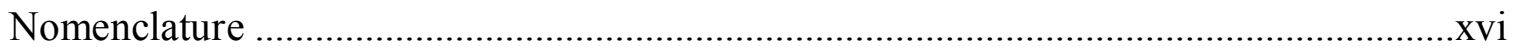

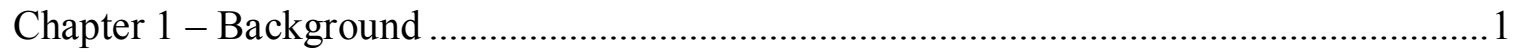

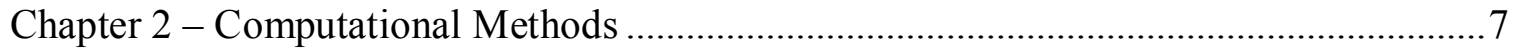

2.1 Flow Solver and Convergence Methods ..........................................................

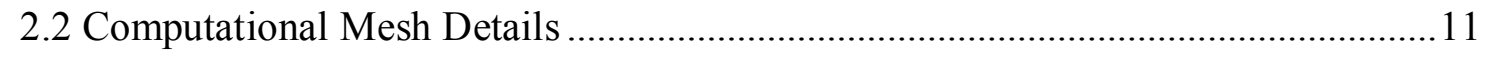

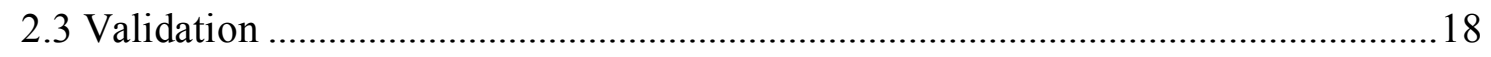




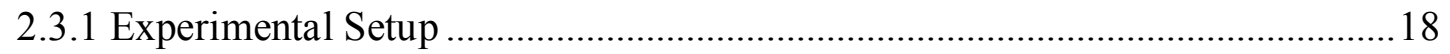

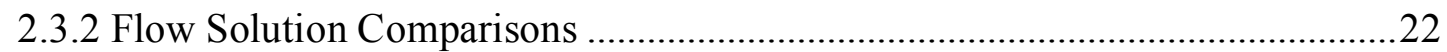

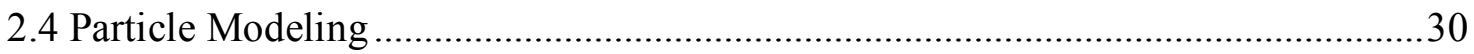

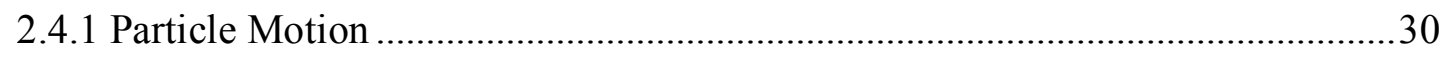

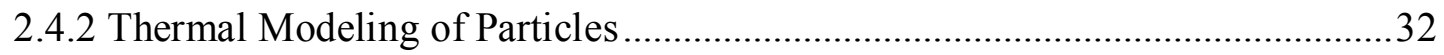

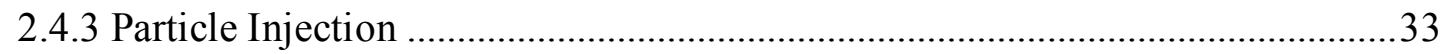

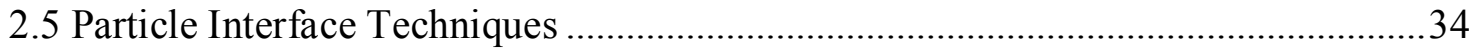

2.5.1 Mixing Plane, Averaged Method …………………………………….............

2.5.2 Mixing Plane, Preserved Method ......................................................................

2.5.3 Sliding Mesh Particle Technique …………………………………................39

2.6 Critical Viscosity Deposition Model ......................................................................

2.6.1 Model Tuning Process ………………………………...................................42

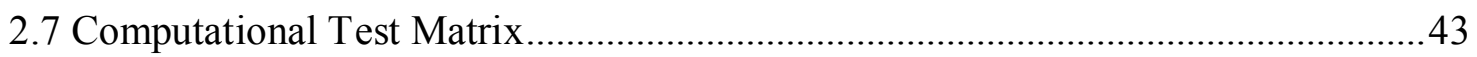

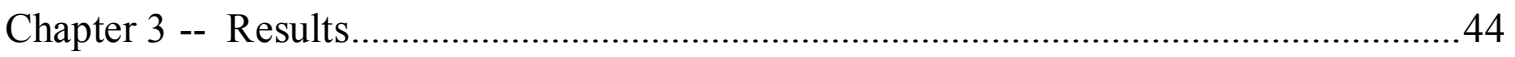

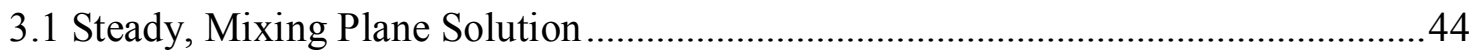

3.1.1 Steady Particle Motion......................................................................................46

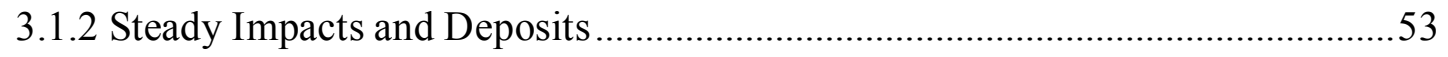

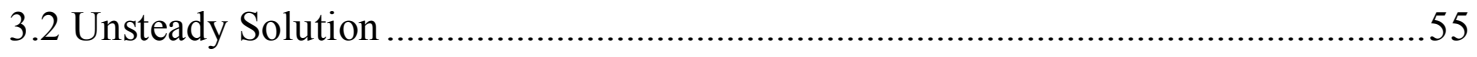

3.2.1 Unsteady Particle Motion, 3 micron particle size ……………….......................58 
3.2.2 Unsteady Impacts and Deposits, 3 micron

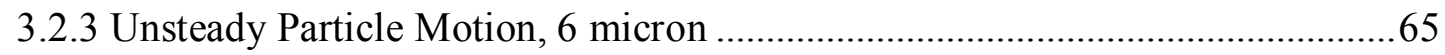

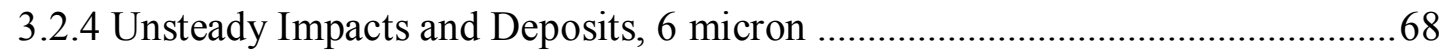

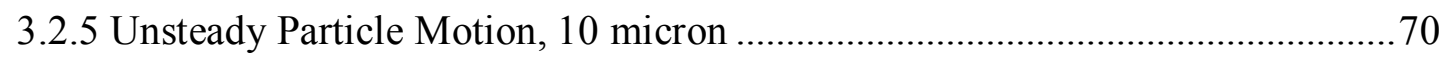

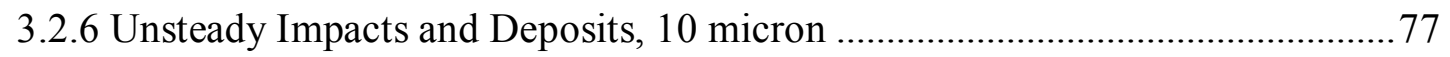

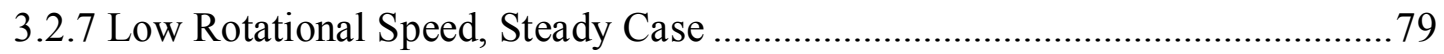

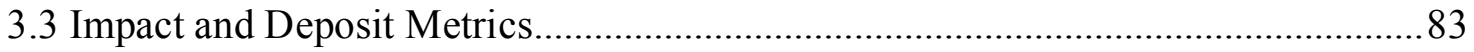

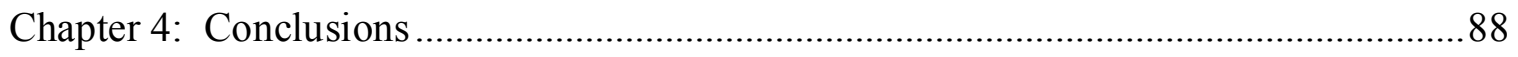

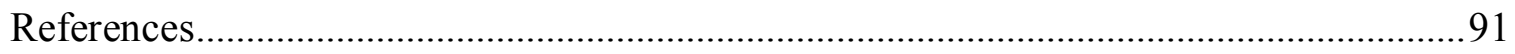

Appendix A - FLUENT User-Defined Function Code for Random Particle Injection ....95

Appendix B - FLUENT User-Defined Function Code for Writing Particle Positions at

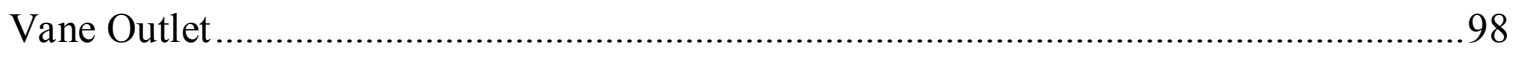

Appendix C - FLUENT User-Defined Function Code for Preserved Mixing Plane Re-

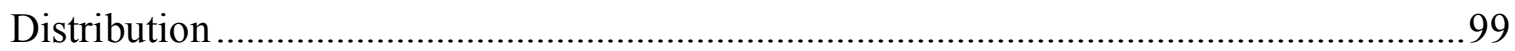

Appendix D - FLUENT User-Defined Function Code for Random Particle Injection and

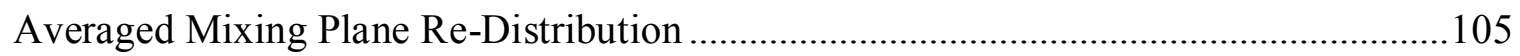

Appendix E: Averaged Mixing Plane Particle Redistribution Results ...........................114

Appendix F - Particle Tracking Troubleshooting Chronology ......................................116

ix 


\section{List of Tables}

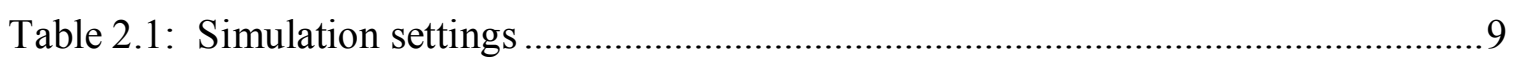

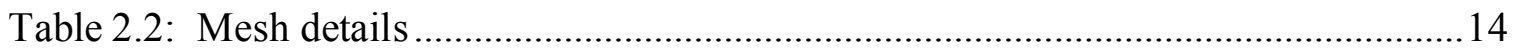

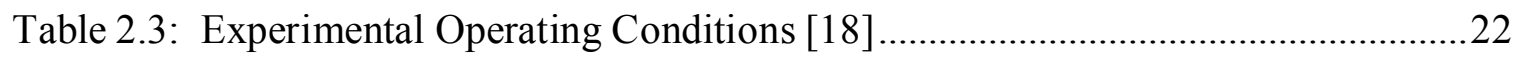

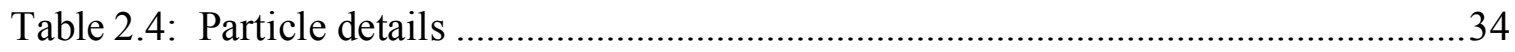

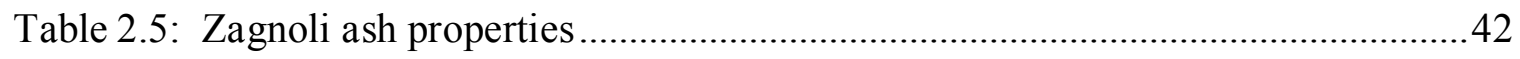

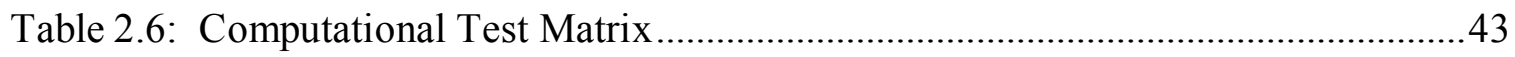




\section{List of Figures}

Figure 1.1: Global particulate concentrations showing heavily affected regions [1] .........1

Figure 2.1: Midspan normalized surface pressure for 36 and 38 blade counts [22] ........12

Figure 2.2: Comparison of medium and fine mesh loadings for steady, mixing plane ....13

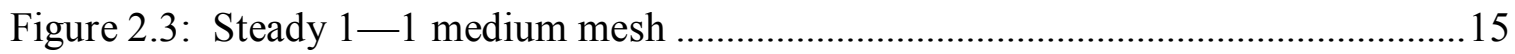

Figure 2.4: Steady $1-1$ medium mesh with boundary settings and mixing plane .........15

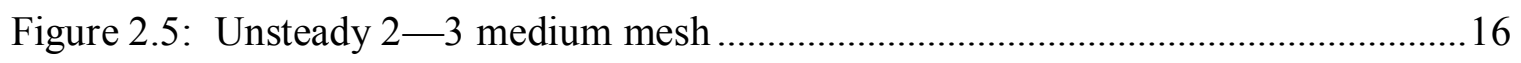

Figure 2.6: Unsteady $2-3$ mesh with boundary settings and sliding interface ...............17

Figure 2.7: Honeywell HPT Experimental Stage [18] .............................................. 19

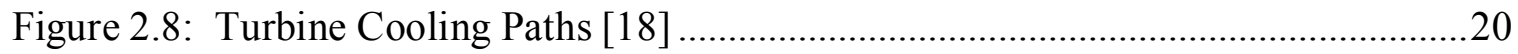

Figure 2.9: Blade surface pressure transducers (50\% and 90\% span) [18] ....................21

Figure 2.10: Constant radius midspan normalized pressure, experimental and numerical

Figure 2.11: Steady mixing plane flow solution-Mach number contours at constant

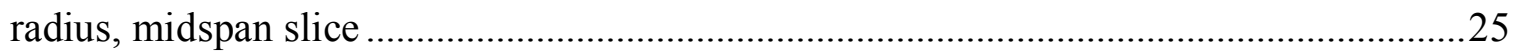

Figure 2.12: Steady, mixing plane solution, close-up leading edge expansion fan ..........26

Figure 2.13: Unsteady midspan, constant radius mach contours, close-up critical region, $\mathrm{t}$

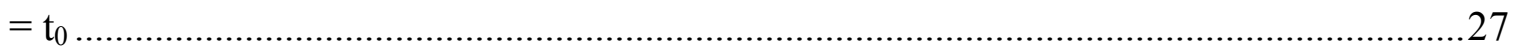


Figure 2.14: Unsteady pressure trace comparison at $-27 \% \mathrm{WD}$, midspan on blade pressure surface [18]

Figure 2.15: Example particle tracks for particles through a non-redistributed mixing plane .35

Figure 2.16: Example particle tracks for particles through a redistributed mixing plane. 36 Figure 2.17: Mixing plane particle average; before and after averaging and random circumferential distribution

Figure 2.18: Particle tracks colored by residence time for $3 \mu \mathrm{m}$ particles, steady mixing plane solution

Figure 3.1: Deposit contours and particle tracks (colored by particle velocity magnitude) at $6 \mu \mathrm{m}$ for averaged and preserved methods .45

Figure 3.2: Rotating forces experienced by particles at midspan as a function of tangential particle velocity 48 Figure 3.3: Preserved method particle tracks colored by velocity magnitude, (a) $3 \mu \mathrm{m}$, (b) $6 \mu \mathrm{m}$, (c) $10 \mu \mathrm{m}$, (d) $15 \mu \mathrm{m}$ .49

Figure 3.4: Relative and absolute Mach contours on constant radial slices, (a) hub region $30 \%$ span, (b) midspan, (c) case region $70 \%$ span. .50

Figure 3.5: Vane wake and particle locations on mixing plane for $6 \mu \mathrm{m}$, vane outlet. Total pressure contours on $50 \%$ span surface.

Figure 3.6: Particle locations at vane outlet for steady mixing plane solutions and particles of diameter (a) $3 \mu \mathrm{m}$, (b) $6 \mu \mathrm{m}$, (c) $10 \mu \mathrm{m}$, (d) $15 \mu \mathrm{m}$ .52 
Figure 3.7: Preserved impact and deposit contours for (a) $3 \mu \mathrm{m}$, (b) $6 \mu \mathrm{m}$, (c) $10 \mu \mathrm{m}$, (d) $15 \mu \mathrm{m}$ .54

Figure 3.8: Midspan absolute and relative total pressure contours at (i) $t=t_{0}$, (ii). .56

Figure 3.9: Velocity triangle schematics for free stream and wake fluid with averaged velocity values

Figure 3.10: Midspan, unsteady $3 \mu \mathrm{m}$ particle locations and wakes at (i) $t=\Delta t+t_{0}$, (ii) $t$ $=6 \Delta \mathrm{t}+\mathrm{t}_{0}$, (iii) $\mathrm{t}=11 \Delta \mathrm{t}+\mathrm{t}_{0}$, (iv) $\mathrm{t}=16 \Delta \mathrm{t}+\mathrm{t}_{0},(\mathrm{v}) \mathrm{t}=21 \Delta \mathrm{t}+\mathrm{t}_{0},\left(\right.$ vi) $\mathrm{t}=26 \Delta \mathrm{t}+\mathrm{t}_{0}$, (vii) $\mathrm{t}=$ $31 \Delta \mathrm{t}+\mathrm{t}_{0}$ .60

Figure 3.11: Unsteady $3 \mu \mathrm{m}$ blade PS impacts at (i) $\mathrm{t}=\mathrm{t}_{0}$, (ii) $\mathrm{t}=16 \Delta \mathrm{t}+\mathrm{t}_{0}$, (iii) $\mathrm{t}=32 \Delta \mathrm{t}$

$+\mathrm{t}_{0}(\mathrm{iv}) \mathrm{t}=48 \Delta \mathrm{t}+\mathrm{t}_{0}$

Figure 3.12: Tip gap (97-100\% span), unsteady $3 \mu \mathrm{m}$ particle locations and wakes at (i) $\mathrm{t}$ $=\Delta \mathrm{t}+\mathrm{t}_{0}$, (ii) $\mathrm{t}=6 \Delta \mathrm{t}+\mathrm{t}_{0}, \quad$ (iii) $\mathrm{t}=11 \Delta \mathrm{t}+\mathrm{t}_{0}$, (iv) $\mathrm{t}=16 \Delta \mathrm{t}+\mathrm{t}_{0},(\mathrm{v}) \mathrm{t}=21 \Delta \mathrm{t}+\mathrm{t}_{0,}$, vi) $\mathrm{t}=$ $26 \Delta \mathrm{t}+\mathrm{t}_{0},\left(\right.$ vii) $\mathrm{t}=31 \Delta \mathrm{t}+\mathrm{t}_{0}$

Figure 3.13: Aggregate impact and capture efficiency contours for $3 \mu \mathrm{m}$ particles (a) steady, (b) unsteady. 64

Figure 3.14: Midspan, unsteady $6 \mu \mathrm{m}$ particle locations and wakes at (i) $\mathrm{t}=\Delta \mathrm{t}+\mathrm{t}_{0}$, (ii) $\mathrm{t}$

$$
\begin{aligned}
& =6 \Delta \mathrm{t}+\mathrm{t}_{0}, \quad \text { (iii) } \mathrm{t}=11 \Delta \mathrm{t}+\mathrm{t}_{0},\left(\text { iv) } \mathrm{t}=16 \Delta \mathrm{t}+\mathrm{t}_{0},(\mathrm{v}) \mathrm{t}=21 \Delta \mathrm{t}+\mathrm{t}_{0},(\mathrm{vi}) \mathrm{t}=\right. \\
& 26 \Delta \mathrm{t}+\mathrm{t}_{0},\left(\text { vii) } \mathrm{t}=31 \Delta \mathrm{t}+\mathrm{t}_{0}\right.
\end{aligned}
$$

Figure 3.15: Tip gap (97-100\% span), unsteady $6 \mu \mathrm{m}$ particle locations at (i) $\mathrm{t}=\Delta \mathrm{t}+\mathrm{t}_{0}$,

$$
\begin{aligned}
& \text { (ii) } \mathrm{t}=6 \Delta \mathrm{t}+\mathrm{t}_{0}, \quad \text { (iii) } \mathrm{t}=11 \Delta \mathrm{t}+\mathrm{t}_{0} \text {, (iv) } \mathrm{t}=16 \Delta \mathrm{t}+\mathrm{t}_{0},(\mathrm{v}) \mathrm{t}=21 \Delta \mathrm{t}+\mathrm{t}_{0} \text {, (vi) } \mathrm{t}= \\
& 26 \Delta \mathrm{t}+\mathrm{t}_{0},\left(\text { vii) } \mathrm{t}=31 \Delta \mathrm{t}+\mathrm{t}_{0}\right.
\end{aligned}
$$


Figure 3.16: Aggregate PS impact and capture efficiency contours for $6 \mu \mathrm{m}$ particles (a) steady, (b) unsteady

Figure 3.17: Aggregate SS impact and capture efficiency contours, $6 \mu \mathrm{m}$ unsteady .......69

Figure 3.18: Midspan, unsteady $10 \mu \mathrm{m}$ particle locations and wakes at (i) $\mathrm{t}=\Delta \mathrm{t}+\mathrm{t}_{0}$, (ii) $\mathrm{t}$ $=6 \Delta \mathrm{t}+\mathrm{t}_{0}, \quad$ (iii) $\mathrm{t}=11 \Delta \mathrm{t}+\mathrm{t}_{0,}$ (iv) $\mathrm{t}=16 \Delta \mathrm{t}+\mathrm{t}_{0},(\mathrm{v}) \mathrm{t}=21 \Delta \mathrm{t}+\mathrm{t}_{0,}$ (vi) $\mathrm{t}=$

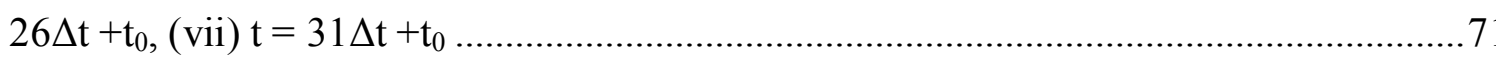

Figure 3.19: Midspan Mach contours (a) steady, and unsteady at (i) $t=\Delta t+t_{0}$, (ii) $t=$

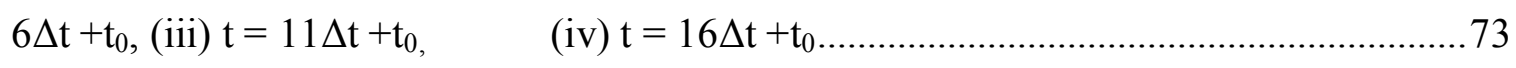
Figure 3.20: Steady, 10 micron particle pathlines colored by particle velocity magnitude

Figure 3.21: Tip gap (97-100\% span), unsteady $10 \mu \mathrm{m}$ particle locations at $(\mathrm{i}) \mathrm{t}=\Delta \mathrm{t}+\mathrm{t}_{0}$, (ii) $\mathrm{t}=6 \Delta \mathrm{t}+\mathrm{t}_{0}, \quad$ (iii) $\mathrm{t}=11 \Delta \mathrm{t}+\mathrm{t}_{0}$, (iv) $\mathrm{t}=16 \Delta \mathrm{t}+\mathrm{t}_{0},(\mathrm{v}) \mathrm{t}=21 \Delta \mathrm{t}+\mathrm{t}_{0},(\mathrm{vi}) \mathrm{t}=$

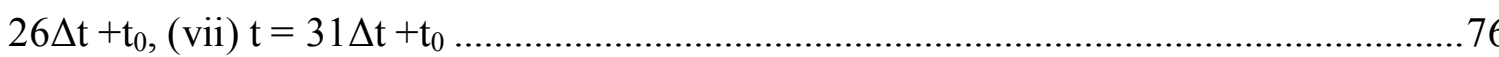

Figure 3.22: Aggregate PS impacts and deposit contours for $10 \mu \mathrm{m}$ particles (a) steady,

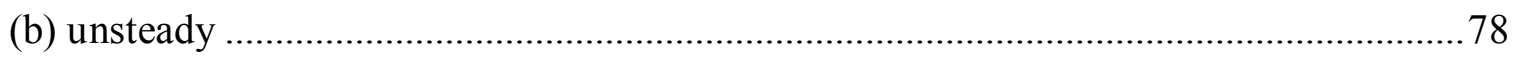

Figure 3.23: Aggregate SS impact and capture efficiency contours, $10 \mu \mathrm{m}$ unsteady .....79

Figure 3.24: Example velocity triangles for two different blade rotational speeds ..........80

Figure 3.25: Particle tracks for (a) $1274 \mathrm{rad} / \mathrm{s}$ and (b) $1000 \mathrm{rad} / \mathrm{s}, 3 \mathrm{micron}$ particles .....81 Figure 3.26: Impact and capture efficiency contours for (a) baseline rotational speed and

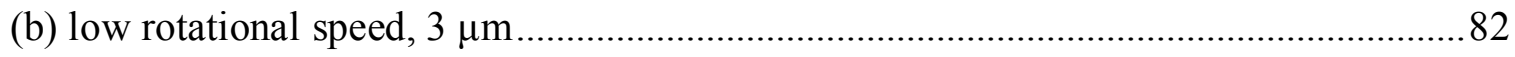

Figure 3.27: Impact and capture efficiencies vs. Stokes number ...................................84

Figure 3.28: Impact and capture efficiencies vs. Stokes number, SS values removed.....87 
Figure E.1: Averaged impact and deposit contours for (a) $3 \mu \mathrm{m}$, (b) $6 \mu \mathrm{m}$, (c) $10 \mu \mathrm{m}$, (d) $15 \mu \mathrm{m}$ .114

Figure E.2: Averaged method particle trajectories for different particle sizes .115 


\section{Nomenclature}

$\begin{aligned} A & =\text { Constant in the Critical Viscosity Model } \\ B & =\text { Constant in the Critical Viscosity Model } \\ C & =\text { Constant in the Critical Viscosity Model } \\ d_{p} & =\text { Particle diameter } \\ h_{\text {inlet }} & =\text { Hydraulic diameter stage inlet } \\ F_{D} & =\text { Drag force per unit mass } \\ M a & =\text { Absolute Mach number } \\ M a_{r e l} & =\text { Rotor relative Mach number } \\ M M D & =\text { Mass mean diameter } \\ \varepsilon_{v} & =\text { Impact efficiency for vane } \\ \varepsilon_{b} & =\text { Impact efficiency for blade } \\ \eta_{v} & =\text { Capture efficiency for vane } \\ \eta_{b} & =\text { Capture efficiency for blade } \\ P & =\text { Static pressure } \\ X & =\text { Axial distance } \\ N_{\text {inj }} & =\text { Number of particles injected } \\ N_{i} & =\text { Number of particles impacted } \\ N_{d} & =\text { Number of particles deposited } \\ P_{\text {exit }} & =\text { Pressure at stage outlet } \\ P t_{\text {inlet }} & =\text { Total pressure at stage inlet } \\ S t_{k} & =\text { Stokes number } \\ T t_{\text {inlet }} & =\text { Total temperature at stage inlet } \\ T u_{\text {inlet }} & =\text { Turbulence intensity at stage inlet } \\ \mu_{\text {crit }} & =\text { Critical viscosity } \\ \mu & =\text { Flow viscosity } \\ \Phi_{m} & =\text { Mass fraction of particles } \\ \Phi_{v} & =\text { Volume fraction of particles } \\ \vec{\omega} & =\text { Blade rotational speed } \\ \vec{r} & =\text { Position vector in rotating frame } \\ \vec{u} & =\text { Flow velocity } \\ & \end{aligned}$




$\begin{aligned} \overrightarrow{u_{r}} & =\text { Flow velocity in rotating frame } \\ \overrightarrow{u_{p}} & =\text { Particle velocity } \\ \vec{g} & =\text { Gravitational constant } \\ \mu_{T p} & =\text { Particle viscosity } \\ \overrightarrow{F_{A}} & =\text { Rotational forces } \\ R e_{p} & =\text { Particle Reynolds number } \\ \rho & =\text { Flow density } \\ \rho_{p} & =\text { Particle density } \\ N_{\text {rotor }} & =\text { Rotor RPM } \\ W D & =\text { Wetted distance } \\ P S & =\text { Pressure surface } \\ S S & =\text { Suction surface } \\ \omega & =\text { Rotating frame rotational speed } \\ m_{p} & =\text { Mass of particle } \\ T_{p} & =\text { Temperature of particle } \\ c_{p} & =\text { Specific heat of particle } \\ h & =\text { Convective heat transfer coefficient } \\ A_{p} & =\text { Surface area of particle } \\ T_{\infty} & =\text { Flow temperature } \\ P r & =\text { Prandtl number of fluid } \\ N u & =\text { Particle Nusselt number } \\ S S T & =\text { Shear Stress Transport }\end{aligned}$




\section{Chapter 1 - Background}

Deposition in gas turbines is a significant problem due to the use of coal-derived synthesis fuels in power generation and rapidly expanding aircraft fleets in areas of high particulate concentration [1].

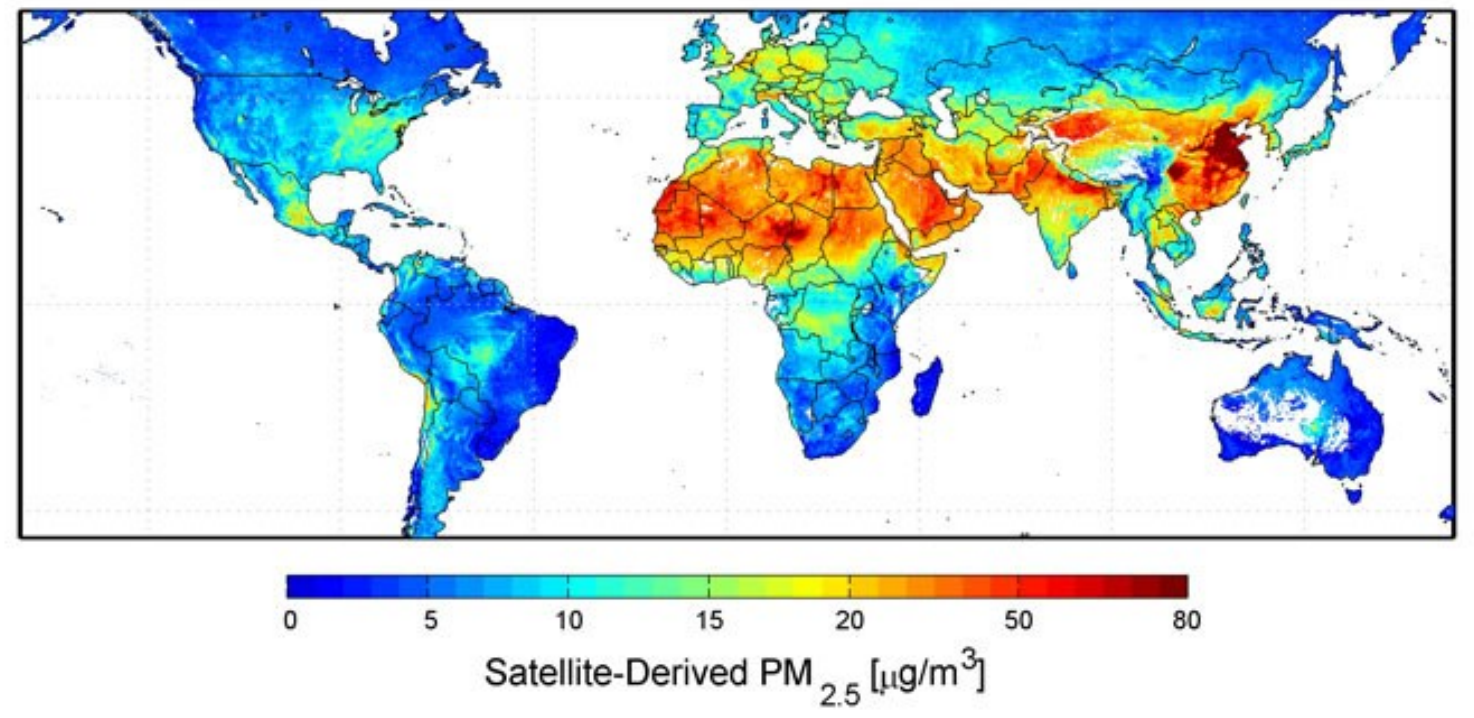

Figure 1.1: Global particulate concentrations showing heavily affected regions [1]

In these scenarios, small particles of ash, sand, or other materials are ingested by gas turbines, heated, and deposited on component surfaces causing major operational problems. Bons [2] found that small-scale deposition can increase the surface roughness of turbine hardware, thus increasing heat transfer to the components. 
Dunn et al. [3] determined that large-scale deposition can harm turbine parts by clogging film cooling holes. They found that deposition resulting from volcanic ash was dependent on turbine inlet temperature, particulate concentration, and material properties of the ingested particulate. The removal and repair of parts affected by deposition is costly and time consuming. The first stage nozzle guide vane and rotor blade of a gas turbine are critically susceptible to deposition as they are subjected to high temperature particleladen flow from the combustor [4]. These conditions make the first turbine stage a very active area of research.

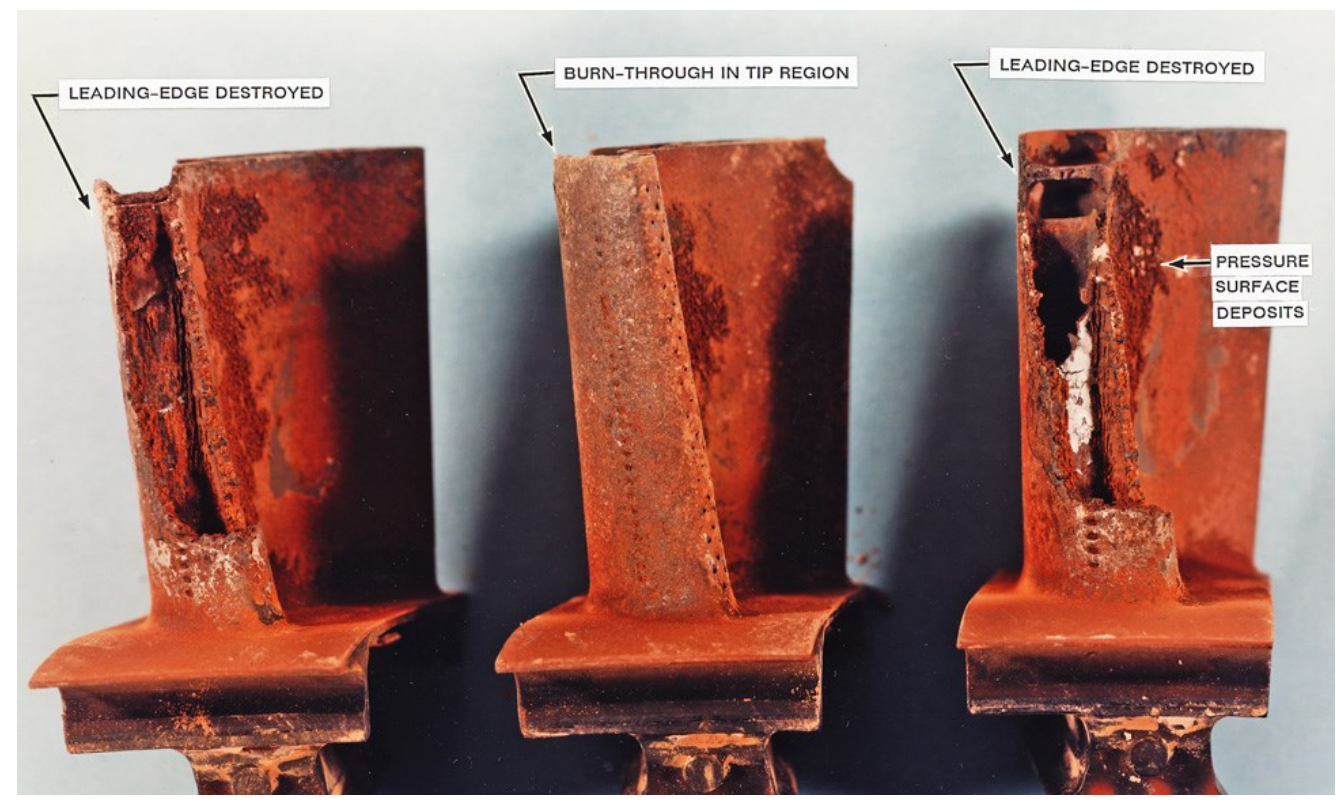

Figure 1.2: Damage to turbine blading due to deposition [4]

In order to numerically characterize and predict deposition in turbomachinery, one must track particles through both the stationary frame of the vane domain and the rotating frame of the blade domain. Several computational studies have been performed in past years tracking different types of particles through a stator and rotor stage. These studies 
include considerable literature on icing and erosion in compressors and erosion in turbines. Tabakoff et al. [5] numerically studied the effect of particle size distribution on blade erosion in an axial flow turbine for particles from 2.5 to $135 \mu \mathrm{m}$. Larger diameter particles, which have larger Stokes numbers, were found to follow ballistic trajectories and deviate from fluid streamlines to impact blade surfaces more often. Larger particles were also found to centrifuge and migrate toward the blade tip more quickly than smaller particles due to their increased mass. Overall, non-uniform impacts were found spreading over most of the blade pressure surface with particle size playing a large role on the severity of erosion. Uniform particle distributions produced localized impact regions near the blade tips, while non-uniform particle distributions produced diffuse impacts over the pressure surface. Tabakoff et al. employed a mixing plane at the interface between stators and rotors allowing for a steady calculation with particle circumferential locations randomly redistributed at the entrance to the rotor in order to simulate the relative motion between successive blade rows. Particle velocities and temperatures were preserved as particles passed from stator to rotor domain. Hamed et al [6] numerically studied the effects of turbine blade surface deterioration by erosion for particles from 30-1500 $\mu \mathrm{m}$ and found that the stator leading edge and pressure surface were especially susceptible to particle impacts and erosion. Also, larger particles tended to have more overall stator and rotor impacts and all sizes saw rapid centrifuging towards the radial direction after impacts. Bidwell [7] numerically studied ice particles in a multistage, low pressure compressor and fan and found that increasing particle size caused less mass to enter the core of the engine due to the larger particles' inability to negotiate the 
turn through the fan. Bidwell employed a mixing plane with particle positions, velocities, and temperatures circumferentially averaged before being passed to the next domain. This method is in contrast to the velocity and temperature preservation method used in Tabakoff's work [5].

Erosion studies in past years have included some alternative methods to mixing plane calculations with distributed particles. For example, Suzuki et al. [8] numerically studied sand erosion in an axial compressor stage using an unsteady calculation with temporal grid geometry updates and found that many repeat impacts occur for both rotor and stator blades with the blade tip being the most susceptible to erosion. Ghanaiet [9] numerically studied particle ingestion in a two-stage gas turbine for particles ranging from 1 to 150 $\mu \mathrm{m}$ in size and found that the trailing edge of the pressure surface of first and second stage vanes as well as the leading edges of first and second stage blades were highly susceptible to particle impacts and subsequent erosion. Ghaneiet used a frozen rotor, multiple reference frame model to calculate the interface between stators and rotors. Corsini et al. [10] conducted a numerical simulation of erosion in a draft fan using a frozen-rotor with a cloud tracking model for particle trajectories while varying particle composition and size. Increasing particle size was found to group particle impacts into localized areas near the tip and leading edge of the draft fan with small particles having more dispersed impact patterns. Yang and Boulager [11] performed a numerical simulation of erosion in an axial fan by modeling the entire fan annulus with both unsteady and steady solution methods. The steady, mixing plane technique was found to 
overpredict impacts and erosion when compared with the unsteady version by as much as $100 \%$ for $280 \mu \mathrm{m}$ MMD sized particles.

While erosion, icing, and deposition studies use Lagrangian tracking to determine particle trajectories they differ on how particles are treated upon impact. Tafti and Sreedharan [12] developed the critical viscosity sticking model using the composition and properties of coal ash to determine a sticking probability based on the viscosity-temperature relationship. This relationship was modeled by Senior and Srinivasachar [13]. Barker et al. [14] adapted the critical viscosity model in [12] to include the effects of shear on particle detachment. Compared to experimental results in Webb et al [15], Barker et al. [14] determined that their deposition model predicts initial deposition relatively well, but is not valid after large-scale deposits begin to form causing geometry changes. Casaday et al. [16] used the deposition model to investigate the effects of a non-uniform inlet temperature profile on external deposition, and found by comparing to experiments that the model could be tuned to produce more representative results. Suman et al. [17] performed a computational study of deposition in a full stage axial compressor using a multiple reference frame, frozen-rotor approach focused on small particulate that could evade an inlet filter. The percentage of particles that hit the blade surface was found to increase with the diameter of particles in a similar fashion to the variation of Stokes number. Particle impact angle and velocity were found to be critical factors in compressor deposition as faster particles impacting at steeper angles to surfaces deposited more often. 
The present study focuses on investigating deposition in the first stage of a high pressure turbine using both steady and unsteady methods in order to observe differences due to particle size and computational methods. The literature includes investigations of deposition in compressor stages and erosion in turbine stages but to the authors' knowledge no publicly available previous studies, computational or experimental, have touched on deposition in a rotating turbine stage. Boundary conditions for this study were extracted from Haldeman et al [18], who experimentally observed the effects of inlet temperature profiles on cooled and uncooled vane and blade performance. Pressure data from the uncooled, uniform inlet case were used from those experiments to validate the present steady calculations before introducing particles into the domain. Nickol et al. [19] did an unsteady analysis of the same data from Haldeman et al. and the unsteady pressures were used to validate the present unsteady calculations. Deposit comparisons could be made if data become available in the future, however, the thrust of this document is to study the merits of different computational methods rather than particle sticking models. The results from this study could be useful in guiding designers or researchers of turbomachinery in best practices for analyzing designs of critical turbine parts for erosion, icing, or deposition mitigation. 


\section{Chapter 2 - Computational Methods}

\subsection{Flow Solver and Convergence Methods}

Computational Fluid Dynamics calculations were performed using a three-dimensional, compressible, Reynolds Averaged Navier Stokes and Unsteady-Reynolds Averaged Navier Stokes commercial finite volume code, FLUENT ${ }^{\mathrm{TM}}$ 13.0. FLUENT's density based solver was selected in order to efficiently solve the significantly compressible flow and shock activity that occur in the turbine stage. For steady solutions an implicit formulation was used with a Roe Flux Difference Splitting scheme in order to calculate the flux vector [20]. A second order upwind scheme was used for spatial discretization of the flow, while a first order upwind scheme was used for turbulent kinetic energy and specific dissipation rate for turbulence calculations. For unsteady solutions an implicit, second order time discretization was used. Similar to the steady solution, a second order upwind scheme was used for spatial discretization of the flow, while a first order upwind scheme was used for turbulent kinetic energy and specific dissipation rate for turbulence calculations.

Total pressure and total temperature were specified at the inlet to the vane, with static pressure specified at the outlet of the blade. Note that these conditions were extracted from Haldeman et al [18]. All walls were specified as adiabatic and the blade domain was set at a constant rotational speed. The adiabatic assumption has some effect on the temperature distributions of the flow as, in reality, heat is removed from the flow by 
turbine components reducing the overall number of deposited particles. However, to accurately capture this effect cooling of the vanes and blades would need to be modelled, which introduces considerable complexity and is outside the scope of this paper.

The k- $\omega$ SST turbulence model was employed, with turbulence intensity and hydraulic diameter specified at the vane inlet ( $5 \%$ and $0.01 \mathrm{~m}$, respectively). The $\mathrm{k}-\omega \mathrm{SST}$ model was selected from FLUENT's available RANS turbulence models due to its superior performance relative to simpler two-equation models [21]. The k- $\omega$ SST model blends the $\mathrm{k}-\omega$ and $\mathrm{k}-\varepsilon$ models, using $\mathrm{k}-\omega$ for near wall calculations and $\mathrm{k}-\varepsilon$ in the free stream. The model also adds a mean, streamwise velocity gradient to the turbulent viscosity equation in order to incorporate shear stress transport. As described by [21], twoequation models, including $\mathrm{k}-\omega$ and $\mathrm{k}-\varepsilon$, suffer from their inability to predict anisotropy of normal stress components and to account for streamline curvature effects. Algebraic stress models outperform two equation models in terms of modeling anisotropy, however they suffer from numerical instability and poor convergence due to numerical stiffness are most effective in heavily swirling flows. Given the instability experienced in the base flow of the present calculations and the need for heavy tuning of solver parameters, the algebraic stress models were avoided. The k- $\omega$ SST was selected as the best candidate for these simulations. 
Temperature dependent polynomials were specified for the thermal conductivity and specific heat of air, while Sutherland's law was employed to compute viscosity. Table 2.1 summarizes the boundary conditions and settings selected for the simulations.

Table 2.1: Simulation settings

\begin{tabular}{|l|c|}
\hline $\mathrm{Pt}_{\text {inlet }}[\mathrm{Pa}]$ & 445,125 \\
\hline $\mathrm{Tt}_{\text {inlet }}[\mathrm{K}]$ & 443 \\
\hline $\mathrm{Tu}_{\text {inlet }}[\%]$ & 5 \\
\hline $\mathrm{h}_{\text {inlet }}[\mathrm{m}]$ & 0.01 \\
\hline $\mathrm{P}_{\text {exit }}[\mathrm{Pa}]$ & 117,325 \\
\hline$\omega[\mathrm{rad} / \mathrm{s}]$ & 1274 \\
\hline$\Delta \mathrm{t}[\mu \mathrm{s}]$ & 10 \\
\hline
\end{tabular}

Convergence of steady solutions was declared when residuals stabilized after falling at least three orders of magnitude and when fair agreement was found with experimental data. Due to the instability of the computational system, care had to be taken when selecting relaxation factors and Courant numbers for both the steady and unsteady solutions. Specifically, very low, conservative values $(\sim 0.25)$ were selected first and then gradually increased as residuals fell. These values were largely determined by experimentation. However, attempts to deviate from specific values typically resulted in divergence.

The unsteady solutions took a special approach to reach convergence. First, a steady mixing plane solution was obtained using the 2 vane, 3 blade mesh as a solution initialization. Then the unsteady solver was implemented and the interface was switched 
to a sliding mesh. Note that the unsteady solutions required the selection of a time step as shown in Table 2.1. The time step was selected based on the rotational speed of the computations and the blade domain is moved an incremental amount at the end of each time step. Specifically, the time was chosen such that 21 time steps corresponded to one vane passing of the blades. In order to converge the unsteady simulation, iterations were performed at each time step (and each new location of the blade) until residuals became periodic. This required approximately 250 time steps to be performed, or about one half annulus of blade rotation. Once this periodic solution was achieved, particles were introduced. Note that due to the nature of the unsteady calculations the particle and flow solution are solved simultaneously, in contrast to the steady method, where the particle forces and locations are updated at the end of every time step. 


\subsection{Computational Mesh Details}

Three-dimensional RANS simulations were performed on two meshes: steady calculations were performed on a mesh made up of one vane and one blade and unsteady calculations were performed on a sliding mesh made up of two vanes and three blades in order to preserve periodicity. The actual number of vanes and blades in the experiment were 24 and 38, respectively. However in this work, for the purposes of simplification, a 24 to 36 vane/blade count was used to allow a 2 to 3 periodic count. Had the original ratio been used, a 12 to 19 vane/blade count would have been necessary to preserve periodicity for unsteady computations, requiring a very large computational cost. Ameri and Rigby performed calculations using the GlennHT CFD solver with identical boundary conditions for the original and adjusted blade counts. The normalized midspan pressure is shown in Figure 2.1 for both configurations [22, 23]. Lowering the blade count has a very minimal effect on the surface pressures and would cause slightly fewer particle impacts due to $5 \%$ lower blade solidity. Since both steady and unsteady computations employed the same adjusted blade count, the thrust of this study, which is a comparison of methods, is unaffected. 


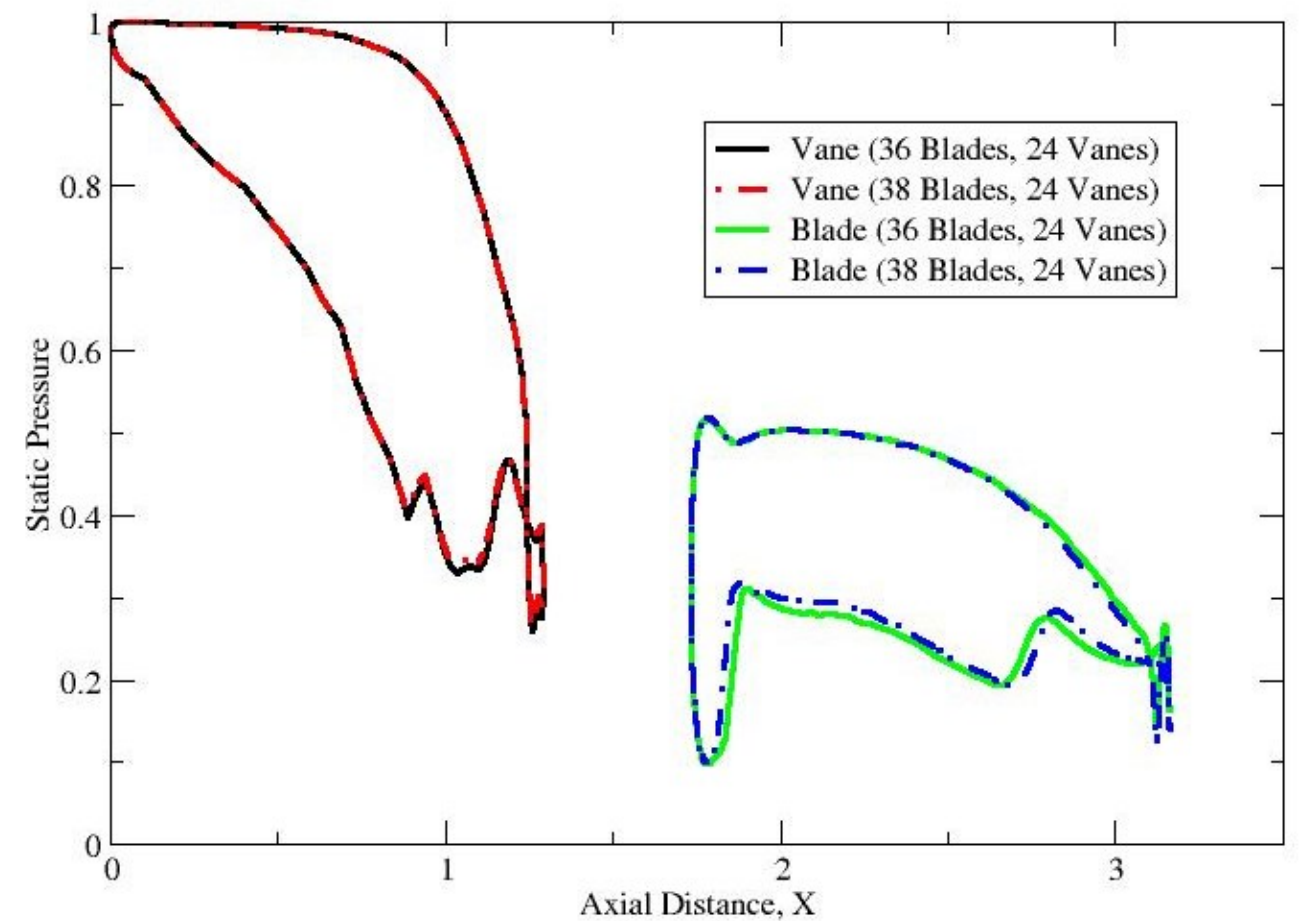

Figure 2.1: Midspan normalized surface pressure for 36 and 38 blade counts [22]

Note also that the steady computations used a single vane and blade and, despite the differences in size of the vane and blade domains, there was no need to adjust the vane/blade count due to the spatial averaging and interpolation performed on the flow by the mixing plane. However, for the sake of comparing the merits of the two computations, the same pitch was used for mixing plane and unsteady computation. Periodic boundary conditions were implemented in both meshes to simulate an annular arrangement. The main inlet of the domain was located approximately $50 \%$ of vane axial chord upstream of the vane leading edge, with the outlet approximately $30 \%$ of vane axial chord downstream from the trailing edge of the rotor. 
Fine and medium meshes were generated using the software GridPro. A fine grid was generated and then coarsened to allow a grid independence study and assessment of the required CPU time for unsteady computations. Figure 2.2 below shows pressure traces taken at midspan on vane and blade surfaces for the medium and fine computational meshes. Both solutions are very similar and capture specific flow features such as shocks near the vane trailing edge and the blade leading edge as evidenced by rapid increases in pressure at those locations. The only recognizable differences between meshes are the locations of the suction peaks on the blade suction surface (at an axial distance of about $x$ =2.25.) Steady mid-span vane and blade surface pressure traces were found to be within $3 \%$ average for the medium and fine meshes.

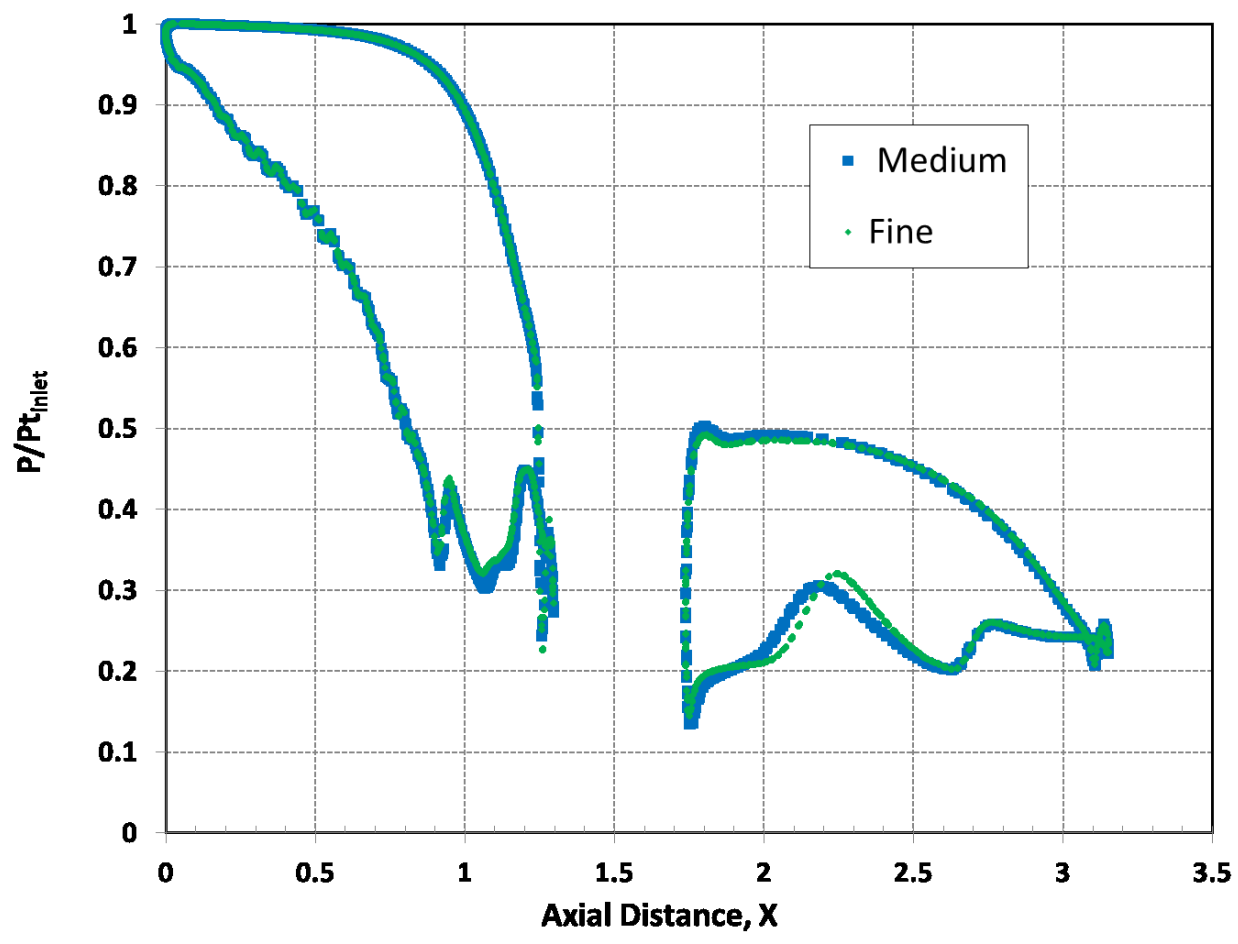

Figure 2.2: Comparison of medium and fine mesh loadings for steady, mixing plane 
Consequently, the medium mesh was used for computations presented in this study at a greatly reduced computational cost. The computational time for the fine mesh was approximately double that of the coarse mesh. The details of each mesh are provided in Table 2.2. Geometry details and boundary assignments are shown in Figure 2.3.

\section{Table 2.2: Mesh details}

\begin{tabular}{|c|c|}
\hline Mesh (\# Vanes - \# Blades) & Number of Cells \\
\hline Medium 1-1 & $1,621,783$ \\
\hline Medium 2-3 & $3,658,637$ \\
\hline Fine $1-1$ & $3,212,240$ \\
\hline Fine $2-3$ & $7,431,764$ \\
\hline
\end{tabular}




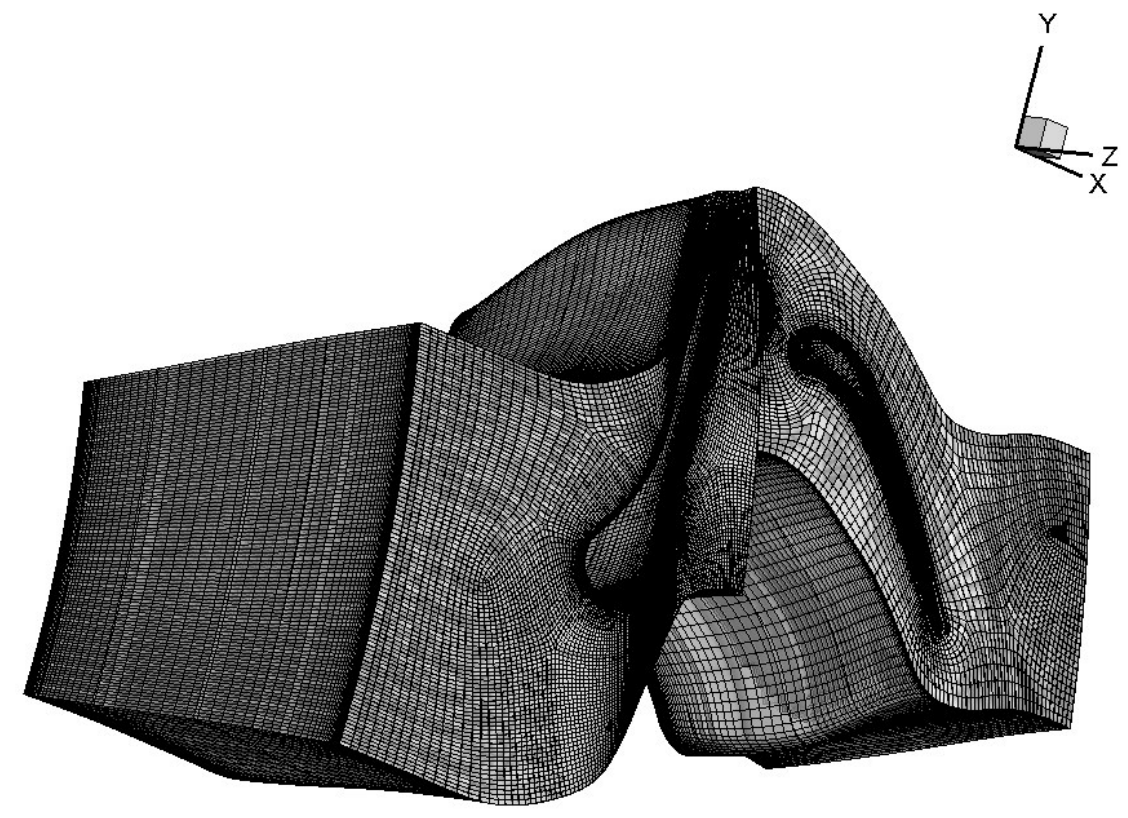

Figure 2.3: Steady 1-1 medium mesh

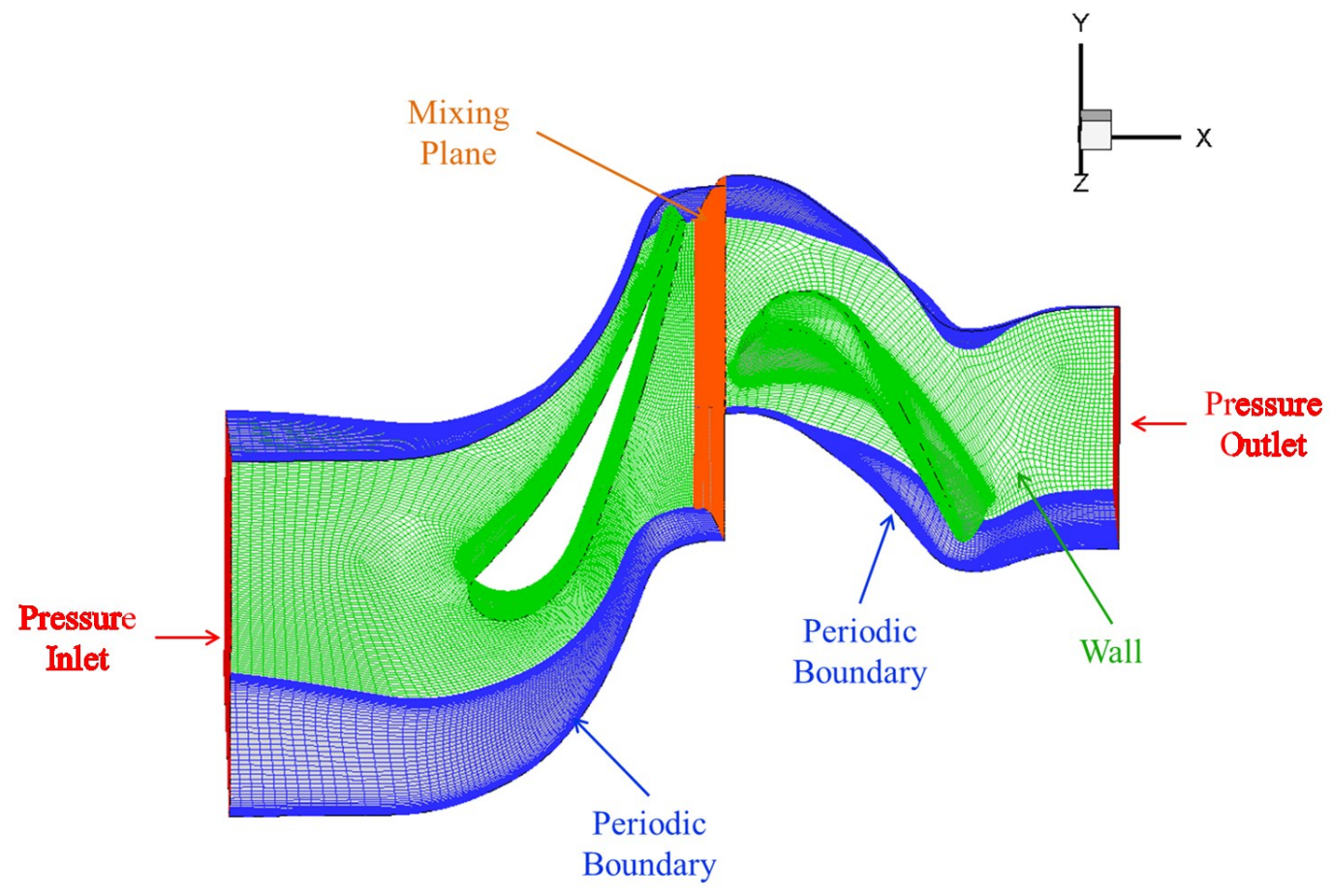

Figure 2.4: Steady 1-1 medium mesh with boundary settings and mixing plane 


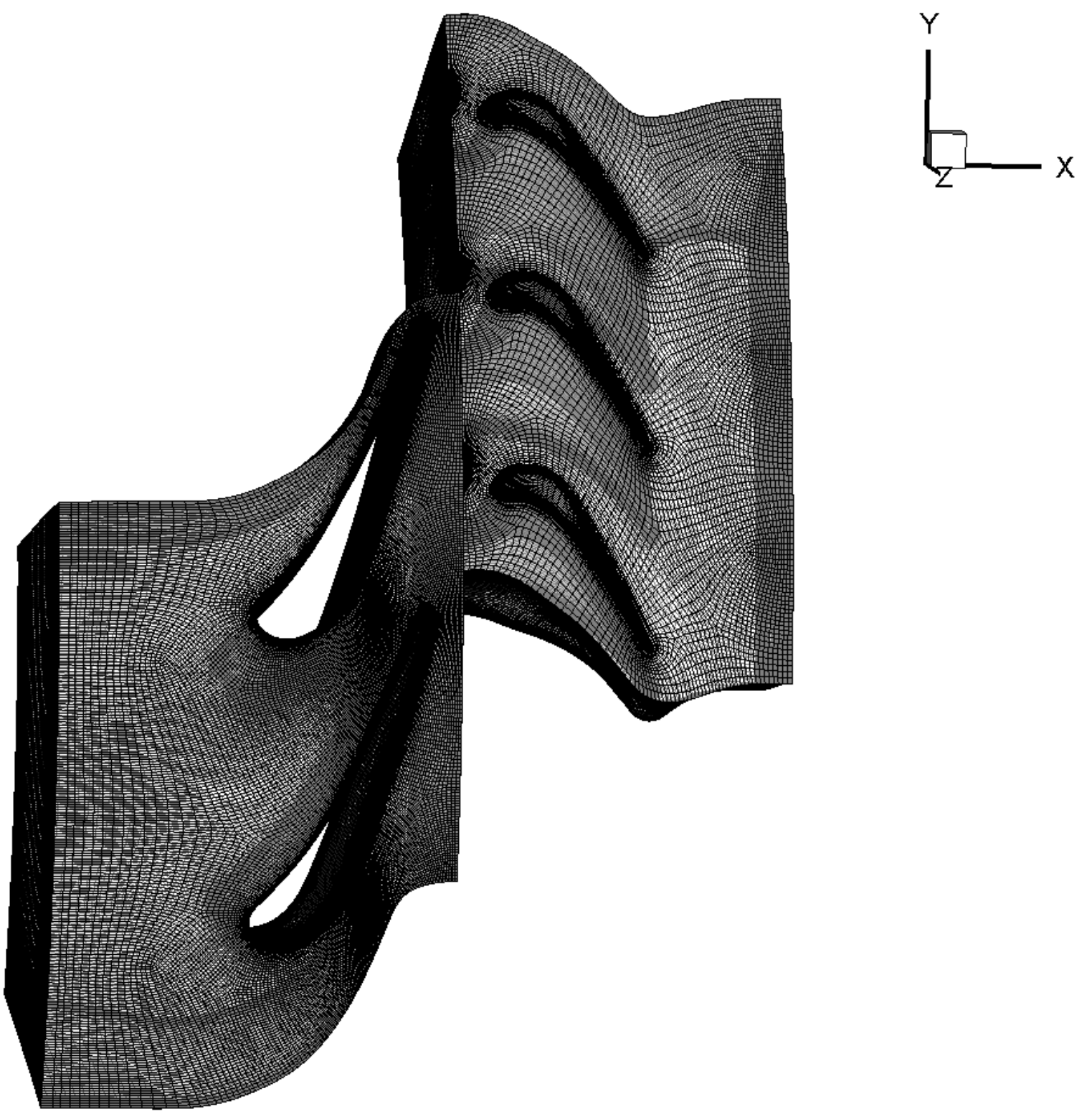

Figure 2.5: Unsteady $2-3$ medium mesh 


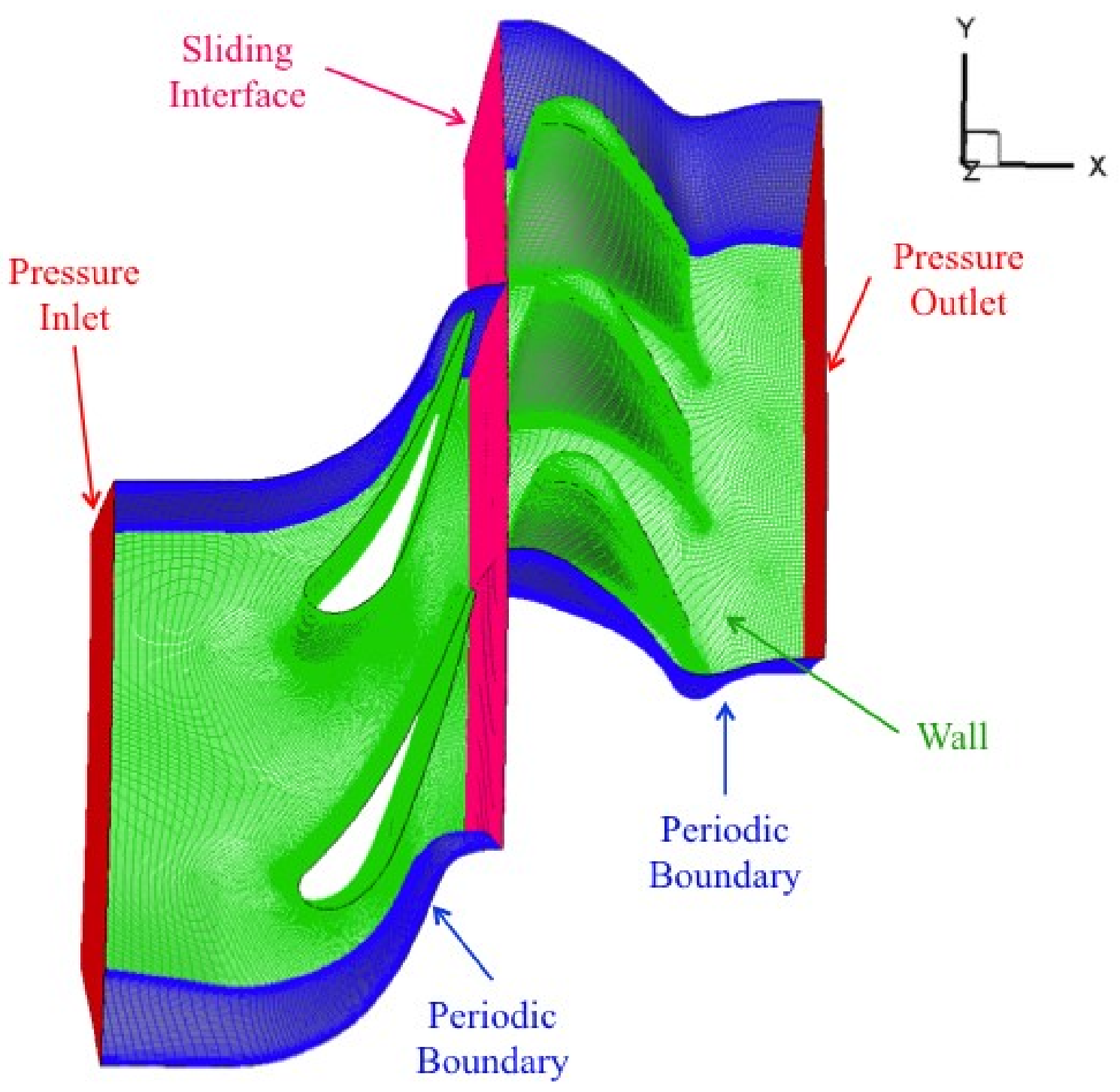

Figure 2.6: Unsteady $2-3$ mesh with boundary settings and sliding interface 


\subsection{Validation}

All flow solutions were validated using experimental data from turbine experiments performed at the Gas Turbine Lab at Ohio State University. The following sections describe the experimental setup in detail and the subsequent comparisons with this study's computational results. They are excerpted from the work of Haldeman et al. and Nickol et al. [18, 19].

\subsubsection{Experimental Setup}

A rotating, blow-down facility including an expansion nozzle, combustor emulator, and full-scale turbine stage was utilized to collect pressure and heat flux data on blade surfaces (Figure 2.7). The expansion nozzle was used to achieve transonic flow through the stage and the aft choke was used to modulate mass flow. Two 300-channel slip rings with 150 pairs of wires each passed data out of the facility to the acquisition system. The combustor emulator used a passive heat exchanger concept to create different inlet temperature distributions with very little total pressure distortion, which is typically experienced with mass flow injection systems. Comparisons were made to the present study's computations for the uniform inlet temperature case only. The combustor emulator acted as a flow straightener and reduced turbulence as an unintended consequence. 


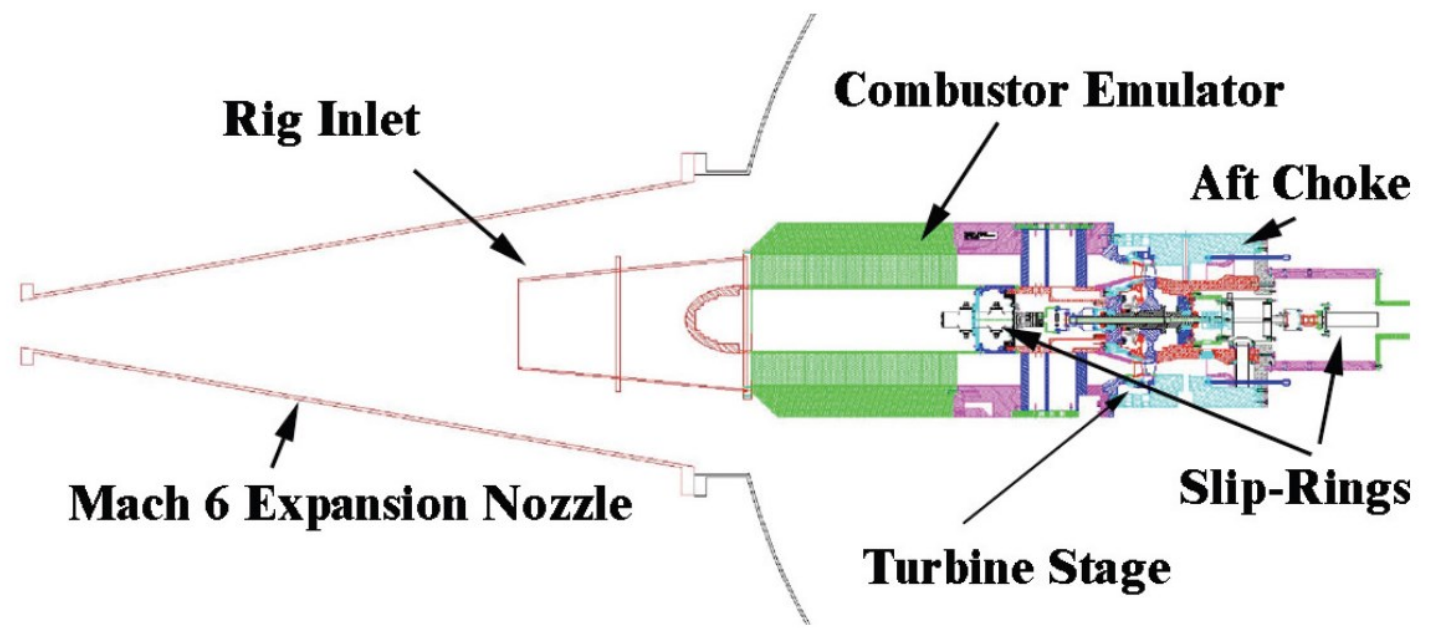

Figure 2.7: Honeywell HPT Experimental Stage [18]

The turbine stage used in the experiments included 12 vane doublets (24 vanes in total) and 38 blades. Note that the ratio of vanes to blades used in the calculations made in the present work (24 vanes and 36 blades) does not match the one used in experiment due to the need to model 19 blades and 12 vanes in order to maintain periodicity. This would require a prohibitively large mesh and computational time would be untenable. This is discussed further in the mesh discussion of Section 2.2. Some of the experimental runs were performed with cooling with coolant supplied to the vanes and blades through struts upstream of the blading. Figure 2.8 shows the paths of the various coolant streams supplied. Note that the present computations did not include any cooling and comparisons were made to the non-cooled experimental cases only. 


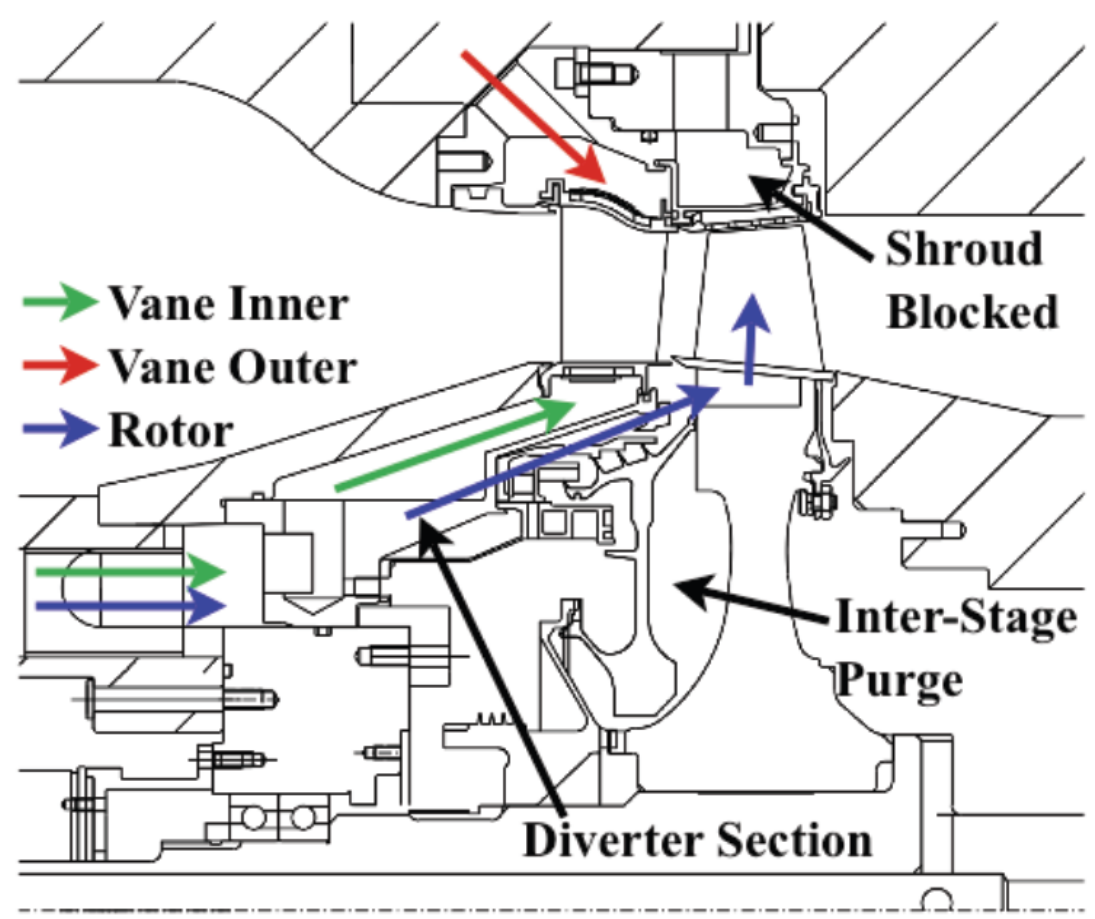

Figure 2.8: Turbine Cooling Paths [18]

The external surfaces of the vanes and blades were instrumented to record pressure and heat flux data in real-time. The vane had 16 external Kulite pressure transducers mounted at 50\% span, while the rotor had 17 identical sensors at 50\% span and 14 at $90 \%$ span. Figure 2.9 shows the blade pressure sensors embedded in the metal surface. 

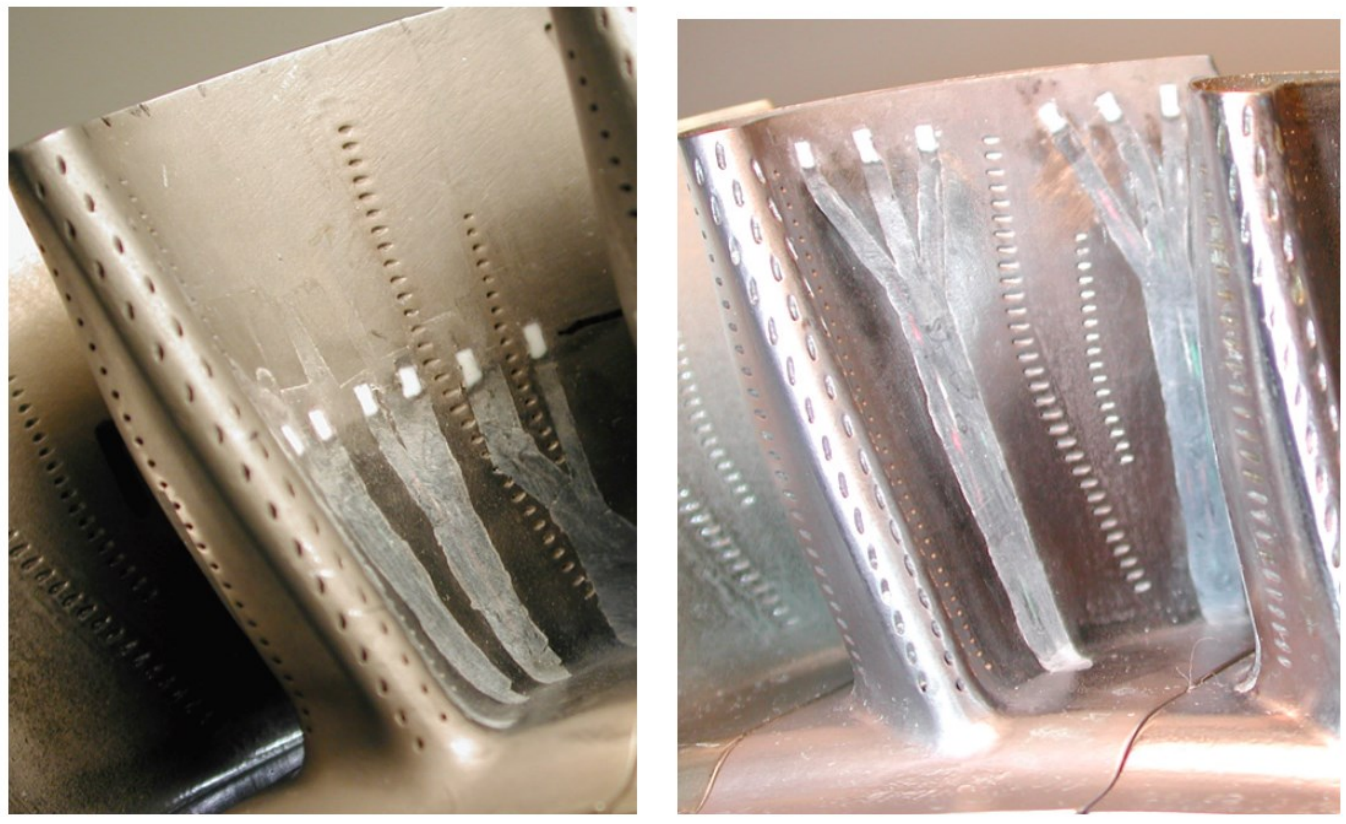

Figure 2.9: Blade surface pressure transducers (50\% and $90 \%$ span) [18]

The white areas pictured are the heads of the sensors, while the grey areas are channels filled with epoxy to secure the sensor leads. The leads run out of the turbine assembly through a slip ring to the data acquisition system. Temperature and pressure rakes are placed at the inlet of the stage in order to characterize inlet distributions. 


\subsubsection{Flow Solution Comparisons}

In order to validate the flow solution of the steady mixing plane calculations, comparisons of pressure ratio were made from the results of the medium $1-1$ mesh to the experimental data of Haldeman et al. [18]. The experimental operating conditions are tabulated below.

Table 2.3: Experimental Operating Conditions [18]

\begin{tabular}{|l|c|}
\hline $\mathrm{Pt}_{\text {inlet }} / \mathrm{P}_{\text {exit }}$ & $3.79 \pm 0.04$ \\
\hline $\mathrm{Tt}_{\text {inlet }}[\mathrm{K}]$ & $443 \pm 1.6 \%$ \\
\hline $\mathrm{N}_{\text {rotor }}[\mathrm{RPM}]$ & $12,165 \pm 0.8 \%$ \\
\hline
\end{tabular}

Figure 2.10 shows midspan pressures on the vane and blade surface normalized by the inlet total pressure as a function of axial distance. Overall the agreement between the experiment and the steady computations is fair. 


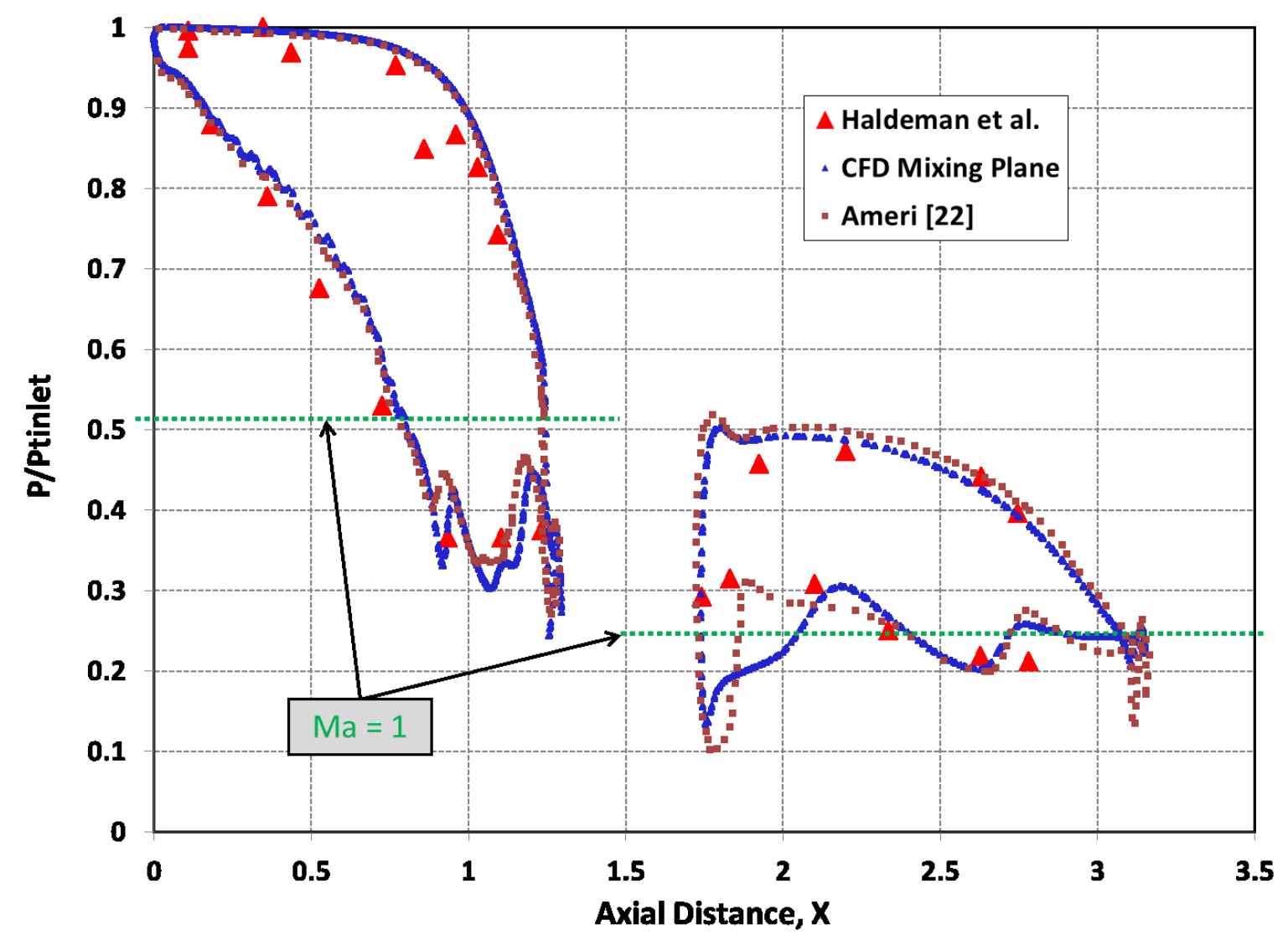

Figure 2.10: Constant radius midspan normalized pressure, experimental and numerical

Note that shocks form at the trailing edge of the vane. These features are captured by the computations and are evidenced by the rapid rise and fall in pressure at these locations as noted in Figure 2.10. Another area of interest is the blade leading edge where the simulations predict a supersonic suction peak. The experimental data are sparse in this location, and, despite not being obvious in the data, that suction peak could exist between the first two pressure taps. The data set colored in red in Figure 2.10 shows another set of calculations performed at the exact same conditions by Ameri and Rigby using the 
GlennHT CFD solver [22, 23]. This data also shows a supersonic suction peak at the blade leading edge residing almost perfectly between the first two pressure tap locations as speculated. Ameri and Rigby's data set gives some confidence in the existence of the supersonic critical region in reality and the validity of the present computations.

Furthermore, even if the supersonic suction peak is only an artifact of the simulations, it occurs in both the steady and unsteady simulations so for the purpose of comparing methods for this paper it is not as critical.

The flow solution is shown in Figure 2.11 through Mach contours on a constant radius midspan slice. Note that the steady flow solution is only solved on one vane and one blade passage but has been duplicated in Figure 2.11 to clarify the periodic boundaries. The figure shows rapid changes in Mach number that are indicative of shocks formed in the vane domains. Specifically, the reader is directed to the oblique shock emanating from the trailing edge of the vane that forms as supersonic passage flow adjusts to the subsonic trailing edge wake. The same shock passes through the periodic boundary and impinges on the suction surface of the adjacent vane. Figure 2.11 also shows the supersonic suction peak region at the leading edge of the blade as flow accelerates over the suction surface. This region is seen in the pressure data in Figure 2.10 as discussed in the previous paragraph. 


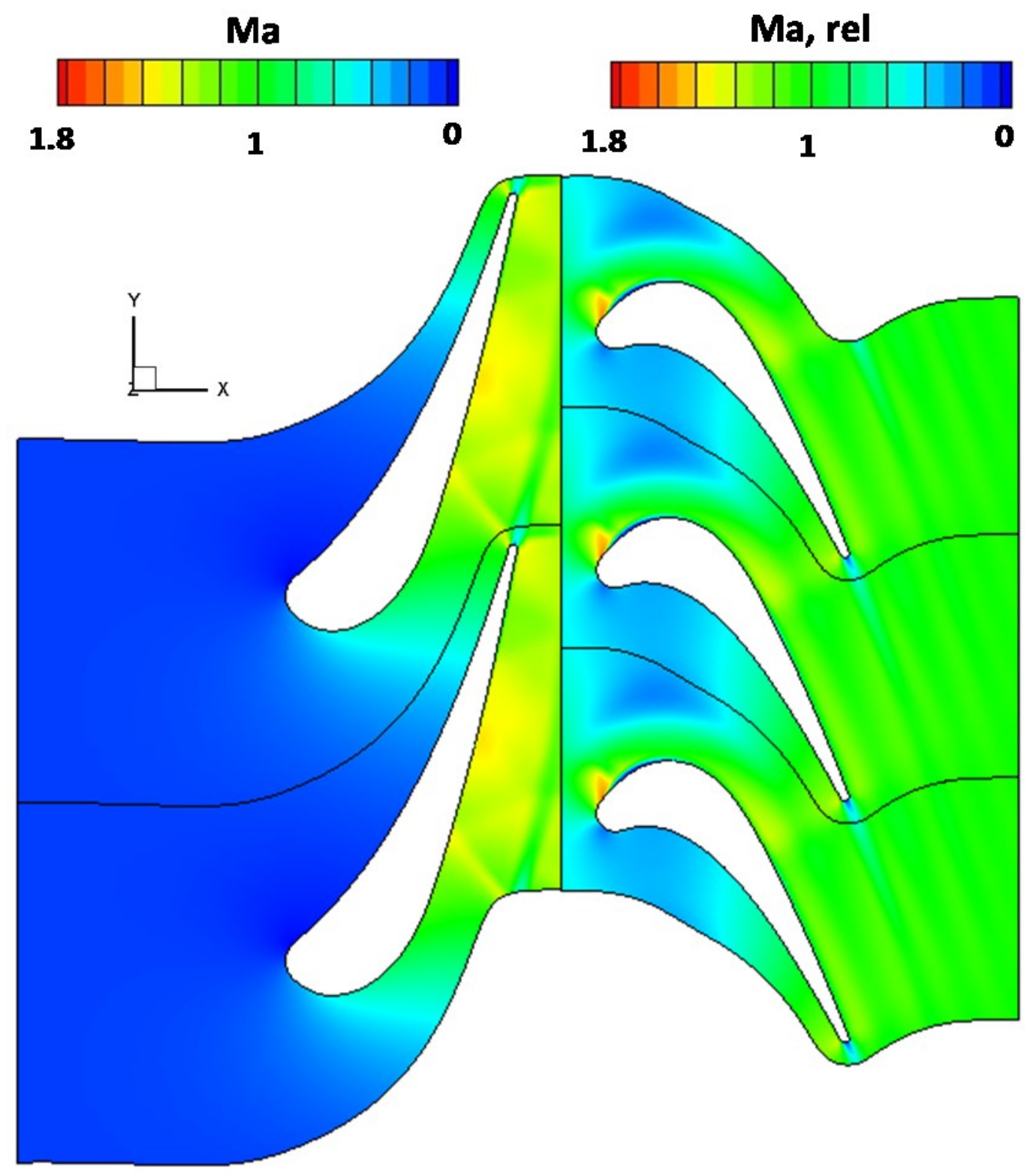

Figure 2.11: Steady mixing plane flow solution-Mach number contours at constant radius, midspan slice 
Figure 2.12 shows a close up of supersonic critical region at the leading edge of the blade. Upon further inspection one can observe what appears to be an expansion fan emanating from the leading edge of the blade, where supersonic flow is expanded around the convex suction side (from $\mathrm{Ma}=1$ to $\mathrm{Ma} \sim 1.6$ ).

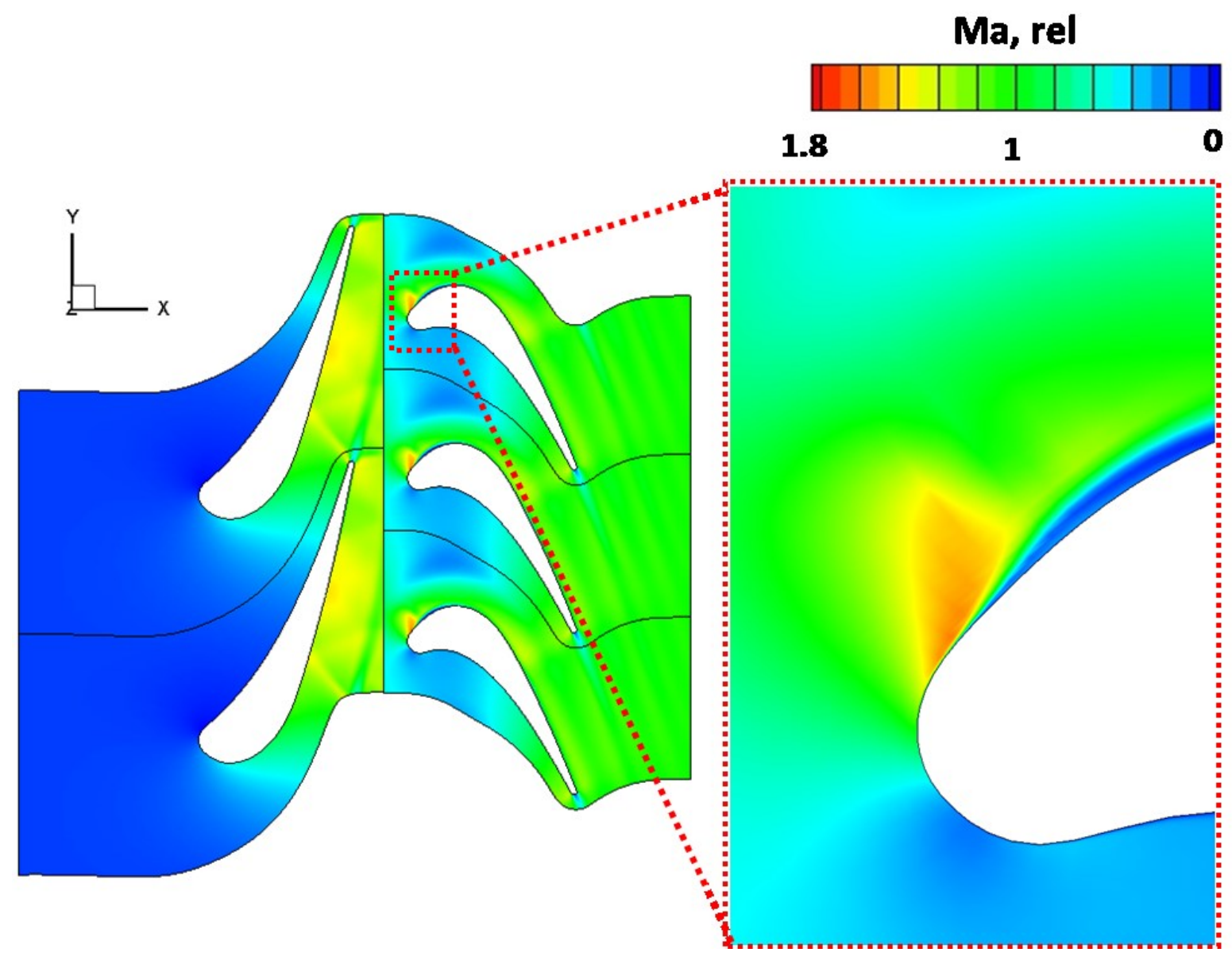

Figure 2.12: Steady, mixing plane solution, close-up leading edge expansion fan

It is important to note the expansion fan formed at the blade leading edge in the steady calculations also occur in the unsteady calculations as shown in Figure 2.13. It also appears that there is a small separation bubble with some recirculating flow in both the 
steady and unsteady methods as is evident by the region of low Mach number fluid just after the expansion fan. Again these occur in both simulations. These similarities give confidence in comparing the two methods in relation to their performance at predicting particle trajectories and deposition.

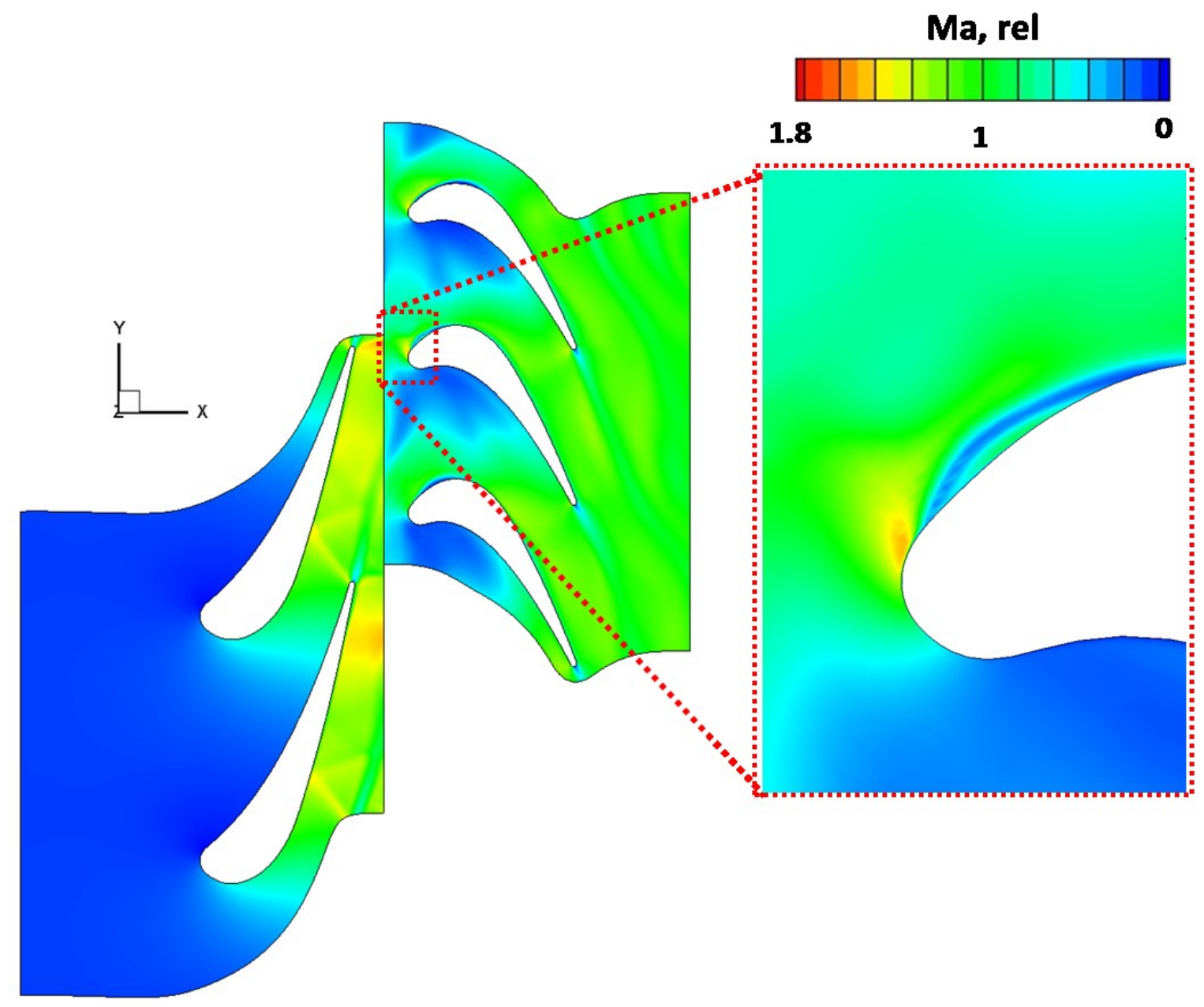

Figure 2.13: Unsteady midspan, constant radius mach contours, close-up critical region, $t=t_{0}$ 
Unsteady pressure data were collected by Haldeman at $-27 \%$ wetted distance (WD) along the pressure surface of the blade [18]. The location is shown in the schematic in Figure 2.14 and unsteady pressure data at that location are plotted against vane passing period. The pressure peaks from the computation are nearly in phase with the experimental data, while the amplitude is within $10 \%$ of the experimental values. The shapes of the pressure traces are also similar. These results are typical for all locations on the blade. Note that the only available time-varying pressure data was for the cooled vane case-all of the present calculations did not use cooling. This mismatch is partly to blame for some of the disagreement between the present computations. The dashed lines in the plot show the time-averaged values for (1) the present CFD simulation (non-cooled), (2) Haldeman experimental data (cooled), (3) Haldeman experimental data (non-cooled). The noncooled time averaged experimental data is significantly lower than that of the cooled case and closer to the average of the present calculations.

Adjustments to the steady and unsteady simulation operating conditions were made in an attempt to improve the agreement with experimental data. These adjustments were done to the rotor rotational speed and inlet total pressures within the bounds of the experimental uncertainty, $\pm 1.03 \%$ and $\pm 1.2 \%$ respectively. These adjustments had little to no effect on the pressure ratios and their agreement to experimental data. The data presented were the best results achieved. 

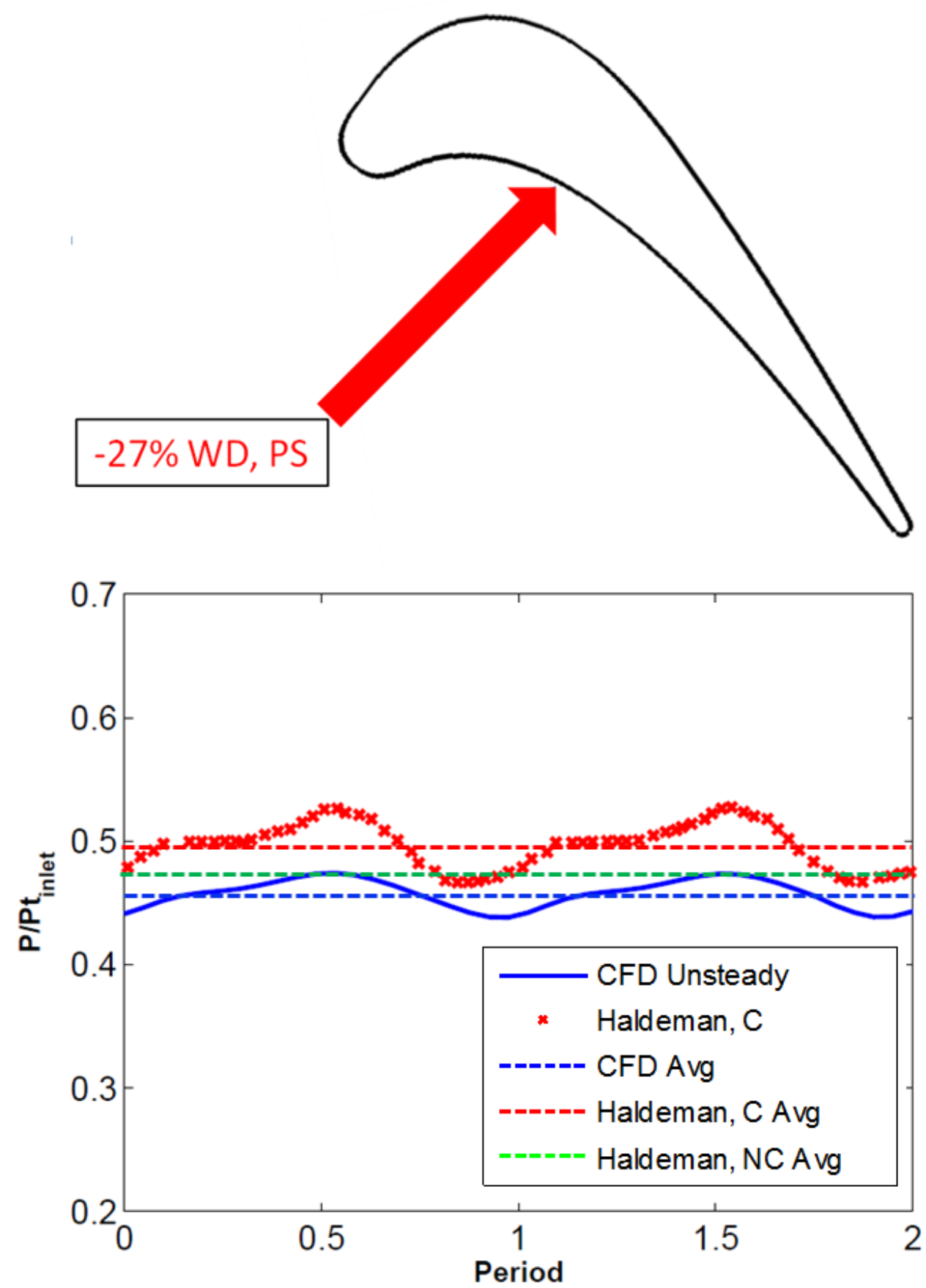

Figure 2.14: Unsteady pressure trace comparison at $-27 \%$ WD, midspan on blade pressure surface [18] 


\subsection{Particle Modeling}

This section describes the equations and techniques used to track particle motion, particle thermal interactions, as well as impact and deposition mechanics.

\subsubsection{Particle Motion}

The Discrete Phase Model (DPM ${ }^{\mathrm{TM}}$ ) included in FLUENT was used to track particles. Due to the low volume fraction of particles used in the calculations, a Lagrangian tracking method was deemed acceptable. This model computes the trajectory of particles using a Lagrangian reference frame that balances the flow induced forces on the particle with the particle's inertia and can be written as

$$
\frac{d \vec{u}_{p}}{d t}=F_{D}\left(\vec{u}-\overrightarrow{u_{p}}\right)+\frac{\vec{g}\left(\rho_{p}-\rho\right)}{\rho_{p}}+\vec{F}_{A}
$$

where $\vec{F}_{A}$ is an additional acceleration term and $F_{D}\left(\vec{u}-\overrightarrow{u_{p}}\right)$ is the drag force per unit particle mass . The drag force per unit mass, assuming particles are spherical and nonrotating, is described as

$$
F_{D}=\frac{3 \mu}{\rho_{P} d_{p}^{2}} \frac{C_{D} R e_{p}}{4}
$$


where $\rho_{P}, d_{p}, u_{p}, \rho, \mu$, and $u$ are particle density, particle diameter, particle velocity, fluid density, fluid viscosity, and fluid velocity, respectively. The particle Reynolds number is described by

$$
R e_{p}=\frac{\rho d_{p}\left|u_{p}-u\right|}{\mu}
$$

The drag coefficient in (2) is described in [26] as

$$
C_{D}=a_{1}+\frac{a_{2}}{R e_{p}}+\frac{a_{3}}{R e_{p}^{2}}
$$

where a1, a2, and a 3 are constants actively adjusted by the Discrete Phase Model tracking algorithm for the valid range of particle Reynolds number [20,24]. Equations (2) and (4) are valid for particle Reynolds numbers up to the turbulent transition value of $10^{5}$, which encompasses the range of the present simulations ( 0 to 500$)$.

The additional acceleration term in (1) can account for "fictitious" forces that occur in the rotating frame like centrifugal and Coriolis forces. These forces must be considered for this application as rotating effects are significant in turbomachinery applications. Rotating forces per unit mass are described by [20],

$$
\overrightarrow{F_{A}}=\left(2 \vec{\omega} \times \overrightarrow{u_{r}}+\vec{\omega} \times \vec{\omega} \times \overrightarrow{\boldsymbol{r}}\right)
$$


where $\vec{\omega}$ is rotational speed, $\overrightarrow{u_{r}}$ is fluid velocity in the rotating frame, $\vec{r}$ and is the position vector in the rotating frame. Other particle forces such as Saffman lift, thermophoresis, Brownian motion, and electromagnetic forces are neglected as they are only significant for sub-micron particles which are smaller than any of the particles for this study [20].

\subsubsection{Thermal Modeling of Particles}

Because the deposition model selected in this paper is primarily dependent on particle temperature, thermal modeling of the particles is required. Equation (1) describes the change in particle temperature in time as a function of the convective heat transfer of the particle with the surrounding fluid (analysis neglects radiation).

$$
m_{p} c_{p} \frac{d T_{p}}{d t}=h A_{p}\left(T_{\infty}-T_{p}\right)
$$

This differential equation is integrated in time to yield the particle temperature [20]. Because the particles are extremely small, their Biot number is less than unity ( $\mathrm{Bi} \sim 0.16$ for the largest particles at max $\mathrm{Re}_{\mathrm{p}}$ ), making a lumped mass assumption appropriate. The lumped mass assumption assumes that the particle does not have significant variations in temperature through its volume; temperature varies in time only. In order to compute the convective heat transfer coefficient to the particle a correlation for Nusselt number is used from Ranz and Marshall [25]:

$$
N u=2.0+0.6 \operatorname{Re}_{p}^{1 / 2} \operatorname{Pr}^{1 / 3}
$$


The Reynolds number is calculated based on the particle diameter as length scale and as the relative velocity between fluid and particle. This correlation is acceptable for small spheres at a large range of Reynolds numbers.

\subsubsection{Particle Injection}

The Eulerian-Lagrangian model separates the particulate modeling into two steps. For steady computations, the flow solution is computed in the first step, absent of particulate. A large number of dispersed particles are then tracked in the second step, each trajectory predicted by computing the local forces at each location along the particle's path. This trajectory is stored for each particle. Typically, for Lagrangian particle tracking problems a random-walk model is used to model dispersion of particles due to turbulent eddies. However, the k- $\omega$ SST model is known to produce unrealistic wall-normal velocity fluctuations within $\mathrm{y}^{+}<10$ of the wall due to its assumption of isotropic turbulence [26]. This error produced unreasonably high impact efficiencies as particles were repeatedly thrown into the wall by the turbulent eddies. Consequently, turbulent dispersion was not implemented in the present study, however, the randomizing effect of the turbulence was somewhat preserved by injecting particles to the vane domain at randomly generated locations for each test case. In these simulations the volume fraction of particles is sufficiently small $\left(\Phi_{V}<10^{-6}\right)$ for all particles sizes such that inter-particle collisions as well as the effect of particles on the flow can be neglected [27]. Table 2.4 shows more details about the diameter, number injected, volume fraction, and mass fraction of particles used in the calculations. 
Table 2.4: Particle details

\begin{tabular}{|c|c|c|c|}
\hline $\mathbf{d}_{\mathbf{p}}[\boldsymbol{\mu \mathbf { m }}]$ & $\mathbf{N}_{\mathbf{i n j}}$ & $\boldsymbol{\Phi}_{\mathbf{v}}$ & $\boldsymbol{\Phi}_{\mathbf{m}}$ \\
\hline 3 & 50,000 & $8.2^{*} 10^{-10}$ & $7.5^{*} 10^{-7}$ \\
\hline 6 & 50,000 & $7.0^{*} 10^{-9}$ & $6.0^{*} 10^{-6}$ \\
\hline 10 & 50,000 & $3.0^{*} 10^{-8}$ & $2.8^{*} 10^{-5}$ \\
\hline 15 & 50,000 & $1.0 * 10^{-7}$ & $9.3 * 10^{-5}$ \\
\hline
\end{tabular}

Once the steady solution converged, the particles were injected at the main inlet boundary upstream of the vane at randomly generated locations, with local flow temperature and velocity using a user defined function. The particles were tracked through the vane passage and coordinates of those not deposited on the vane surfaces were recorded at the exit of the vane domain using another user defined function. Then the particles were redistributed with a random circumferential coordinate and injected into the blade domain using two different methods that will be discussed in the Section 2.5. (User defined function codes for the methods are included in Appendices A-D).

\subsection{Particle Interface Techniques}

Mixing planes are utilized in steady simulations of turbomachinery stages as a means of passing information between rotating and non-rotating components. Flow variables such as total and static pressure, total temperature, and flow orientation are circumferentially mass averaged at the interface plane. Total pressures, total temperatures, and flow angles averaged at the upstream row of the mixing plane are used as the inlet boundary conditions for the downstream row. Circumferentially averaged static pressure from the 
downstream row of the mixing plane is used as the outlet boundary condition for the upstream blade row [20].

Because the flow traverses a mixing plane to pass from the stationary vane domain to the rotating blade domain, methods must be employed in order to simulate the relative clocking of particles with the blade as they enter the rotating frame. If particles are simply allowed to pass from one domain to the other it would simulate the blade being fixed at a certain relative clocking position in a frozen-rotor type simulation.

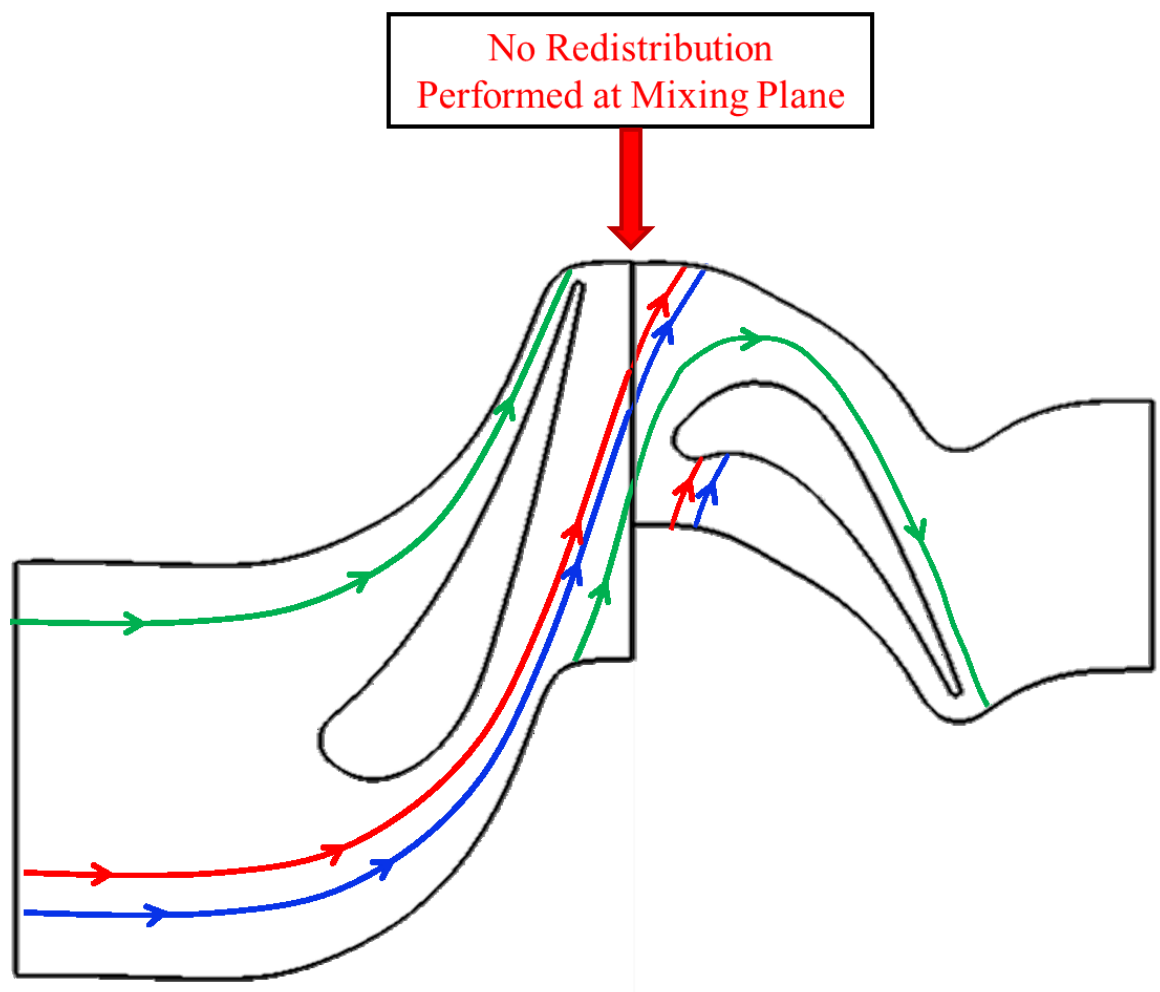

Figure 2.15: Example particle tracks for particles through a non-redistributed mixing plane

While frozen-rotor calculations can be useful and have better convergence and low computational time (Figure 2.15), in order to compare directly to the unsteady 
calculations, where relative particle clocking with the blade is fully preserved, distribution methods are required. The random redistribution of particles produces results like that in Figure 2.16. Note the differences between this configuration and the frozenrotor example in Figure 2.15.

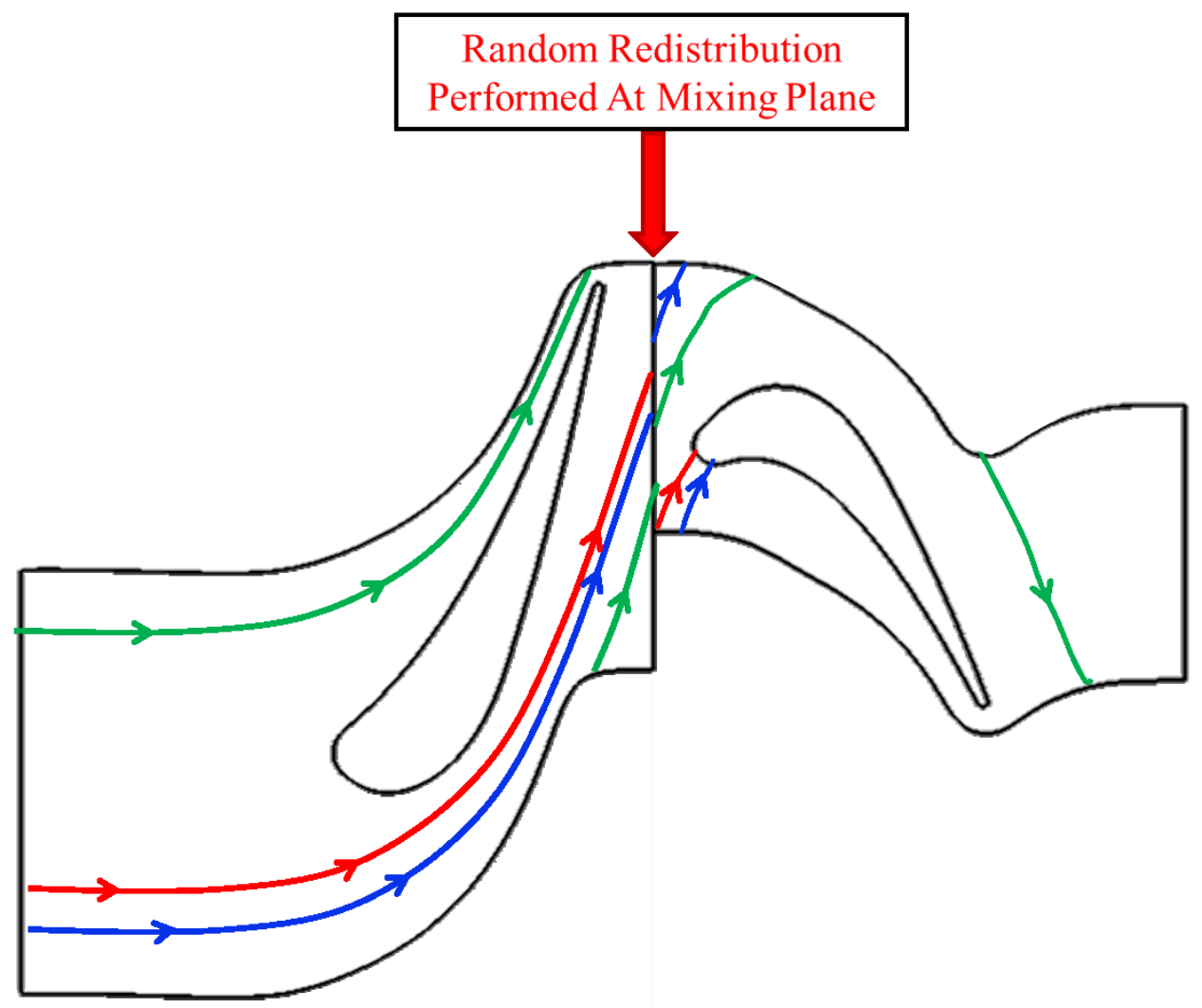

Figure 2.16: Example particle tracks for particles through a redistributed mixing plane. 


\subsubsection{Mixing Plane, Averaged Method}

The first method for particle redistribution broke up the vane exit into radial bands of equal width, averaging all of the particle velocities, temperatures, and diameters in each band and assigning those averaged values to each particle within the respective band. Figure 2.17 demonstrates the process graphically. The left side of the figure shows the locations of particles at the exit of the vane domain. The right side of the figure, in red, shows the particles being redistributed with randomly generated circumferential locations in order to fit the size of the blade inlet and to simulate relative blade clocking. (Note that the velocity vectors and particle temperatures are averaged as well.)
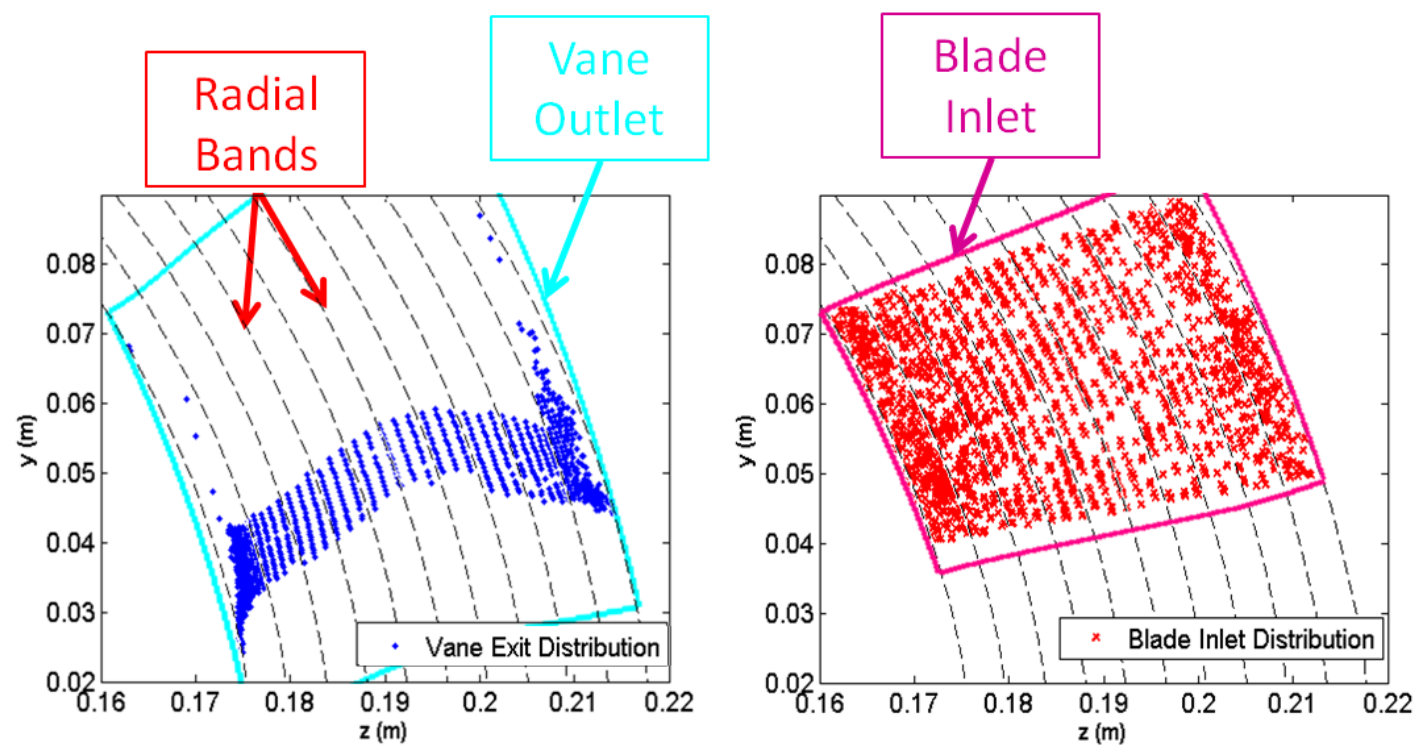

Figure 2.17: Mixing plane particle average; before and after averaging and random circumferential distribution

Once this distribution process was performed the particles were injected into the relative frame of the blade domain. In both the vane and the blade domain, when a particle's 
trajectory was predicted to impact the vane surface, the sticking model was implemented using a user-defined function to determine the sticking probability (Section 2.6). If sticking was not predicted, typical coefficient of restitution data were utilized from experimental data with coal ash data to predict rebounding tangential and normal velocity components (values of 0.4 and 0.8 were used for tangential and normal values, respectively) [28]. Impact and deposit locations are stored and available for analysis, such as determining impact and capture efficiencies, once the simulation is complete.

\subsubsection{Mixing Plane, Preserved Method}

The second method for passing particles through the mixing plane interface preserved each particle's velocity, temperature, and radial coordinate but randomly assigned a new circumferential coordinate as in the averaged method. This method will be referred to as "preserved" throughout the paper. As in the averaged technique in Section 2.5.1, after the distribution process was performed the particles were injected into the relative frame of the blade domain and tracked, while impacts and deposits were recorded for postprocessing. 


\subsubsection{Sliding Mesh Particle Technique}

A different approach was taken for the unsteady simulations: in order to reduce computational time, particles were injected into the steady vane/rotor solution and their locations were recorded at the vane outlet using the same user defined function as previously. The transient vane solution shows $<1 \%$ unsteadiness of the average, as compared to $24 \%$ for the blade domain, so this was deemed a valid approach. The unsteadiness was quantified by the following process: at every location on the vane and blade surfaces along a constant radius at midspan, the standard deviation in time of static pressure was calculated for one vane passing period. Then at every location on the vane and blade surfaces along a constant radius at midspan, the average in time of static pressure was calculated. The standard deviations were then divided by the average at every location giving a deviation in time at every midspan vane and blade location as a percentage of the average values. Then for each surface, these percentages at every midspan location were averaged for each surface giving one percentage value of unsteadiness for each vane and blade.

The steady solution shows particles residing in the vane domain for more than $70 \%$ of the total transit time through the stage so avoiding the unsteady tracking in the vane domain was a $\sim 70 \%$ cost savings. Figure 2.18 shows an example of a Lagrangian particle tracking run performed on the steady, mixing plane flow solution. Note that the flow at the inlet of the vane is relatively slow and requires a large number of time steps to traverse to the vane inlet plane where it is accelerated substantially. 


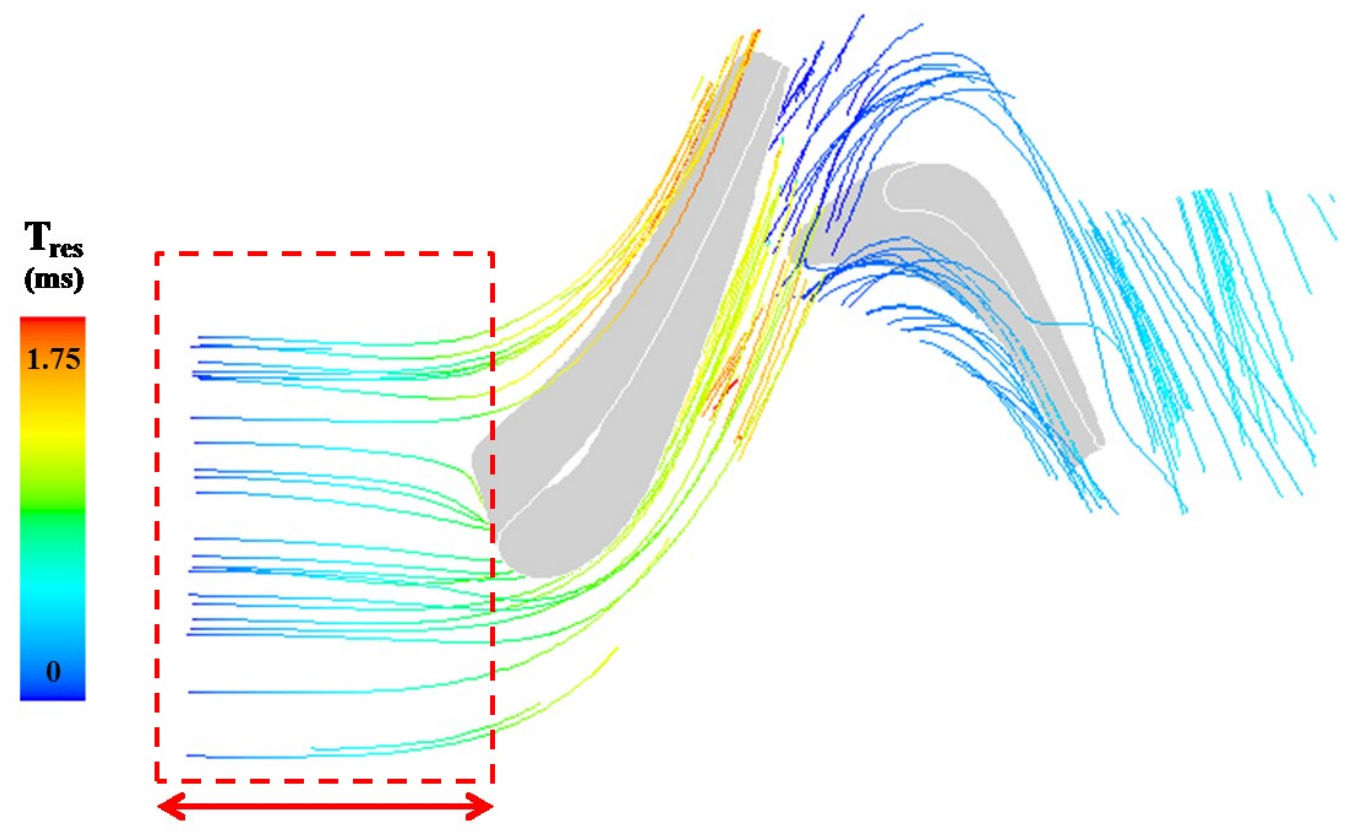

Figure 2.18: Particle tracks colored by residence time for $3 \mu \mathrm{m}$ particles, steady mixing plane solution

With the vane outlet particle locations recorded, injections were made into the unsteady blade domain at the beginning of each time step in accordance with the correct clocking of the rotor in time. Particle trajectories in the rotor are computed based on the forces experienced due to the flow at each time step. Particle locations are then updated with the flow at the completion of a time step. The injections were performed for 21 flow time steps (corresponding to one vane passing period) at which point they ceased. Then the solution was calculated for an additional 63 time steps, giving all of the injected particles the necessary time to fully traverse the rotor domain. 


\subsection{Critical Viscosity Deposition Model}

Tafti and Sreedharan [12] proposed the critical viscosity model, a sticking model based on the particle viscosity. The viscosity is dependent on particle temperature and this dependency can be predicted by examining the properties of the ash. The ash softening temperature is used as the critical sticking temperature, $T_{s}$. Particles with a temperature above this critical temperature are assumed to always stick. Particles with a lower temperature have a probability of sticking based on the ratio,

$$
P_{s}\left(T_{p}\right)=\frac{\mu_{c r i t}}{\mu_{T_{p}}}
$$

where $\mu_{c r i t}$ is the particle viscosity at $T_{S}$ and $\mu_{T_{p}}$ is the particle viscosity at the current particle temperature $T_{p}$. N'Dala et al. [29] developed an expression for the temperature dependence of viscosity for silicate and aluminosilicate,

$$
\log \left(\frac{\mu}{T_{P}}\right)=A+\frac{10^{3} B}{T_{p}}
$$

where A and B are constants obtained from a tuning process. Senior and Srinivasachar [13] conducted experimental tests to develop a curve fit for determining A and B. 


\subsubsection{Model Tuning Process}

Because the experiment by Haldeman et al. was performed at temperatures much lower than those typically found in gas turbines, a tuning process was performed in order to produce a fictitious particle type with constants, A, B, and C, that would deposit in the simulations. The critical viscosity model used was tuned using typical vane capture efficiencies from experiment for coal ash particles at an inlet temperature of approximately $1400 \mathrm{~K}[16]$. By tuning the model parameters to the vane capture efficiencies from high temperature experiments, realistic vane capture efficiencies were achieved in the simulation at an inlet temperature of $443 \mathrm{~K}$. This tuning was assumed to be appropriate for the blade surface as well due to the lack of experimental, rotating blade deposition data available. The main ash properties are summarized in Table 2.5 and described in further detail in [16].

Table 2.5: Zagnoli ash properties

\begin{tabular}{|l|c|}
\hline Density $\left[\mathrm{kg} / \mathrm{m}^{3}\right]$ & 2320 \\
\hline Specific Heat $[\mathrm{J} / \mathrm{kgK}]$ & 984 \\
\hline Thermal Conductivity $[\mathrm{W} / \mathrm{mK}]$ & 0.5 \\
\hline Diameter, $[\mu \mathrm{m}]$ & $3-15$ \\
\hline Stokes Number, $\mathrm{St}_{k}$ & $0.2-5.7$ \\
\hline Viscosity Model Constant, A & -39.85 \\
\hline Viscosity Model Constant, B & 1.172 \\
\hline Viscosity Model Constant, C & 1837 \\
\hline
\end{tabular}




\subsection{Computational Test Matrix}

Several different simulations were performed to constitute the study. Specifically, different steady mixing plane and unsteady sliding interface simulations were completed. The Table 2.6 below documents the different calculations performed. The grid independence calculations are not included since they did not entail any particle tracking or deposition and were for validation purposes only. The $1-1$ and $2-3$ meshes refer to the vane and blade count used for the steady and unsteady simulations. Case 9 refers to a special steady case performed at a lower rotational speed in order to investigate the effect on particle trajectories. The results of all of these cases are discussed in Chapter 3.

Table 2.6: Computational Test Matrix

\begin{tabular}{|c|l|l|l|r|r|}
\hline Case & \multicolumn{1}{|c|}{ Simulation } & Mesh & Interface & \multicolumn{1}{c|}{$\begin{array}{c}\text { Particle } \\
\text { Size } \\
(\boldsymbol{\mu m})\end{array}$} & \multicolumn{1}{c|}{$\begin{array}{c}\text { Rotational } \\
\text { Speed (rad/s) }\end{array}$} \\
\hline 1 & Steady & $1--1$, Medium & MP Averaged & 3 & 1274 \\
\hline 2 & Steady & $1--1$, Medium & MP Averaged & 6 & 1274 \\
\hline 3 & Steady & $1--1$, Medium & MP Averaged & 10 & 1274 \\
\hline 4 & Steady & $1--1$, Medium & MP Averaged & 15 & 1274 \\
\hline 5 & Steady & $1--1$, Medium & MP Preserved & 3 & 1274 \\
\hline 6 & Steady & $1--1$, Medium & MP Preserved & 6 & 1274 \\
\hline 7 & Steady & $1--1$, Medium & MP Preserved & 10 & 1274 \\
\hline 8 & Steady & $1--1$, Medium & MP Preserved & 15 & 1274 \\
\hline 9 & Steady, Low Speed & $1--1$, Medium & MP Preserved & 3 & 1000 \\
\hline 10 & Blade Unsteady & $2--3$, Medium & Sliding & 3 & 1274 \\
\hline 11 & Blade Unsteady & $2--3$, Medium & Sliding & 6 & 1274 \\
\hline 12 & Blade Unsteady & $2--3$, Medium & Sliding & 10 & 1274 \\
\hline
\end{tabular}




\section{Chapter 3 -- Results}

Particle motion, impact, and deposition results were collected for all of the steady and unsteady cases. The steady particle motion is presented first, followed by steady impact and deposit results for all particle sizes. Then unsteady particle motion and impact and deposit results are presented for each particle size individually, followed by aggregate impact and deposit metrics for all cases.

\subsection{Steady, Mixing Plane Solution}

The two mixing plane methods of simulating blade motion relative to particles (averaged and preserved particle data) were found to produce very similar particle trajectories, deposit distributions, and capture efficiencies in the rotor. Figure 3.1 shows particle pathlines and impact locations using the two methods for 6 micron particles. Very little difference is seen in the particle tracks and the impact distributions and, as will be demonstrated quantitatively in Section 3.3, there is very little difference in the impact efficiency and capture efficiency values $(<1 \%)$ at every particle size as well. These similarities are attributed to the fact that most velocity and temperature variations are in the spanwise or radial direction-thus when those quantities are averaged circumferentially it has a minimal effect on the results. Specifically, there is $4 \%$ variation of average velocity in the circumferential direction as compared to $7 \%$ in the radial 
direction. Also, there is $2 \%$ variation of average temperature in the circumferential direction as compared to $4 \%$ in the radial direction. Due to the two methods' similarity, the rest of the results discussed use only the preserved technique with variations in particle size when referring to the steady method for conciseness. Comprehensive particle tracks and impact and deposit results for the averaged technique are shown in Appendix E.

(a)

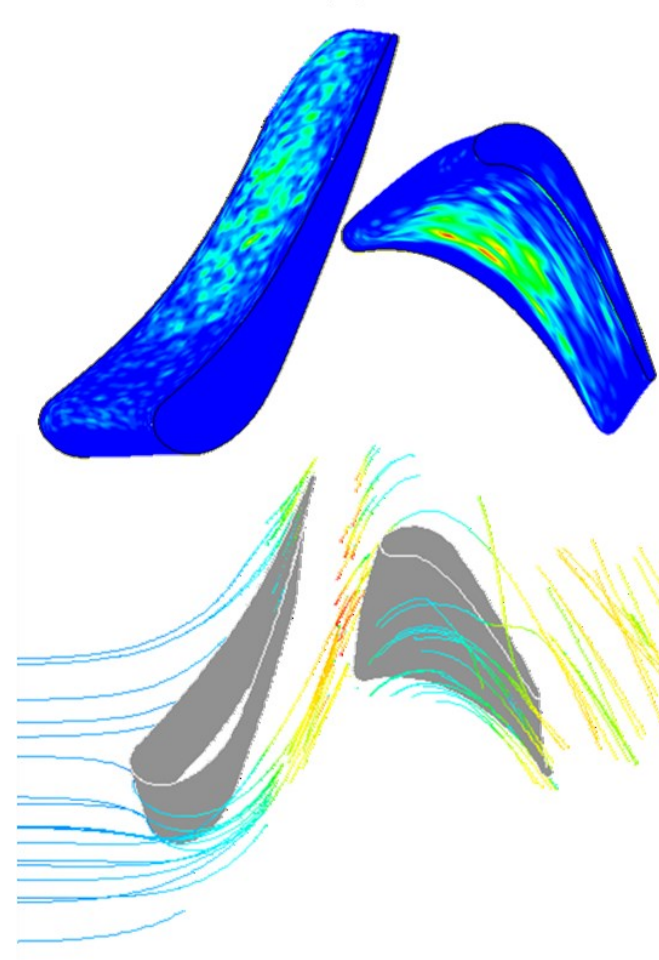

(b)

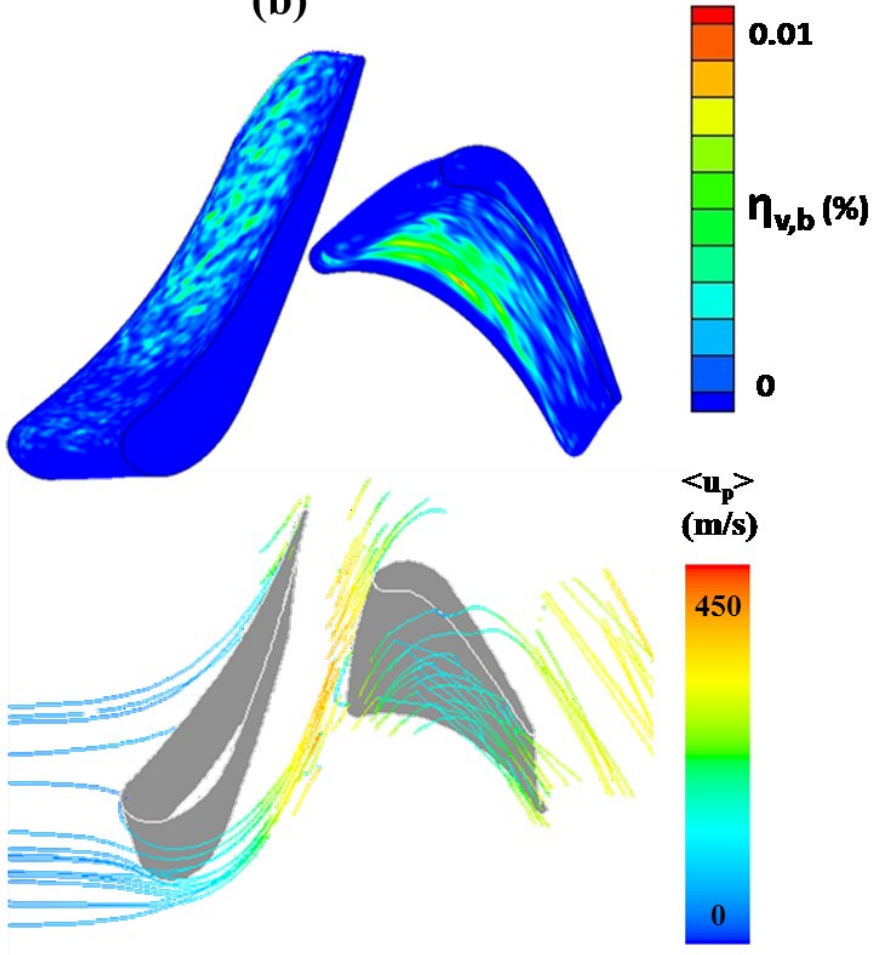

Figure 3.1: Deposit contours and particle tracks (colored by particle velocity magnitude) at $6 \mu \mathrm{m}$ for averaged and preserved methods 


\subsubsection{Steady Particle Motion}

In order to accurately predict impacts and subsequent deposits, the motion of particles must be studied. Plots of particle trajectories show interesting results with respect to particle size: in general the larger particles follow more ballistic trajectories and deviate from streamlines more readily as seen in previous studies in the literature. For example, in the right column of Figure 3.3 particles can be observed separating from the suction peak of the vane, leaving the periodic boundary (where the particle pathlines disappear and reappear), and impacting the pressure surface of the adjacent vane before ricocheting into the blade domain. These impacts on the trailing edge, pressure surface of the vane cause particles to enter the blade domain at a higher relative incidence angle, making them more likely to impact the blade surface. As particle size increases the number of particles separating from the suction surface impacting near the vane trailing edge pressure surface increases.

In the left column of Figure 3.3 one can observe the larger particles migrating towards the tip of the blade. This motion is caused by interplay between two forces experienced by particles in the rotating frame. The first force is a centrifugal force exerted on particles that is proportional to their mass in the rotating frame and is always exerted in the positive radial direction. For example, a particle at the midspan radial coordinate $(\mathrm{r}=$ $0.199 \mathrm{~m}$ ) would experience approximately $3 \times 10^{5} \mathrm{~N} / \mathrm{kg}$ of centrifugal force per unit mass in the positive radial direction. The second force is the Coriolis force which is on 
the order of centrifugal forces. The same particle, having just travelled through the interface between stationary and rotating frame with axial and tangential velocity components of $200 \mathrm{~m} / \mathrm{s}$ (resulting in a typical $280 \mathrm{~m} / \mathrm{s}$ velocity magnitude) would experience $5 \times 10^{5} \mathrm{~N} / \mathrm{kg}$ of Coriolis force per unit mass. Coriolis forces only act on the perpendicular component of particle velocity with respect to the rotational axis. So for particles travelling through the stationary-rotating interface with a positive inlet angle, which indicates a positive tangential velocity component, a negative radial Coriolis force will be experienced (i.e. forcing particles to a smaller radius). However, once particles enter the rotating frame and flow through the blade passage the flow direction changes due to the curvature of the blades. In this region the particles experience a negative tangential component of velocity, resulting in a positive radial Coriolis force (i.e. forcing particles to a larger radius). 


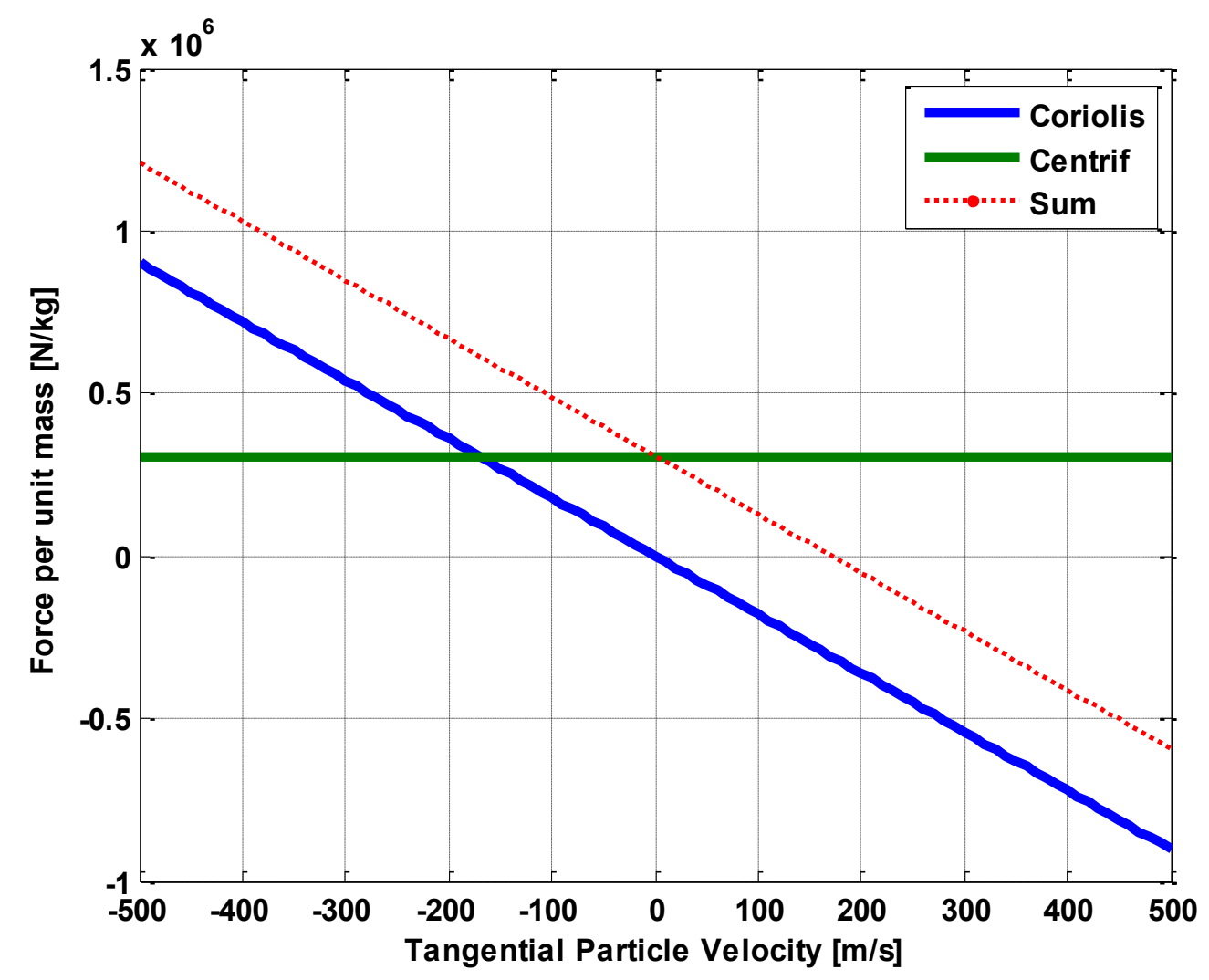

Figure 3.2: Rotating forces experienced by particles at midspan as a function of tangential particle velocity

Figure 3.2 shows the interplay between particle forces due to the change in sign of the particle tangential velocity. Particles at the inlet to the blade (in the $+150-250 \mathrm{~m} / \mathrm{s}$ range) experience opposing centrifugal and Coriolis forces that are of order and largely cancel each other out. Any radial particle motion in that location is not dominantly affected by these forces. However, once the flow direction changes as it follows along the blade surface, resulting in a negative tangential velocity, the centrifugal and Coriolis forces act in the same positive radial direction. This is the region where particles are observed to migrate out on radius, especially for the larger particles as seen in Figure 3.3. Larger 
particles accelerate more slowly and enter the blade domain with a lower velocity, resulting in a smaller Coriolis force. Consequently, larger particles are more dominated by centrifugal forces.

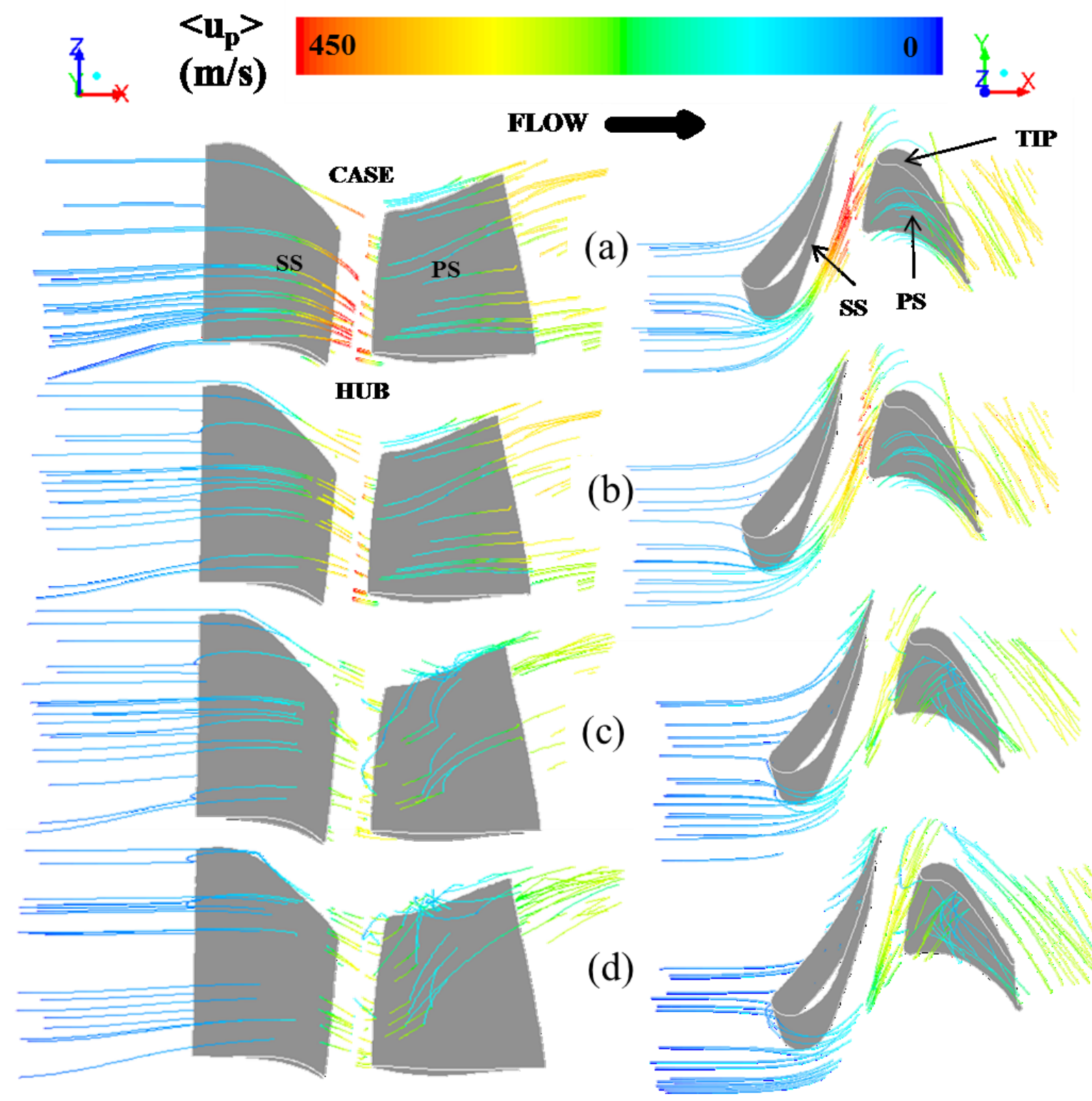

Figure 3.3: Preserved method particle tracks colored by velocity magnitude, (a) $3 \mu \mathrm{m}$, (b) $6 \mu \mathrm{m}$, (c) $10 \mu \mathrm{m}$, (d) $15 \mu \mathrm{m}$ 
Due to the three dimensional nature of the vane structure, the throat is narrower towards the hub side ( $\sim \%$ circumferentially) causing a span wise variation in stream wise acceleration of the flow and entrained particles. Figure 3.4 demonstrates the span wise variations of flow acceleration which result in particles having a higher velocity and a larger incidence angle at the hub entering the blade domain, resulting in many more impacts near the hub region of the blade surface.

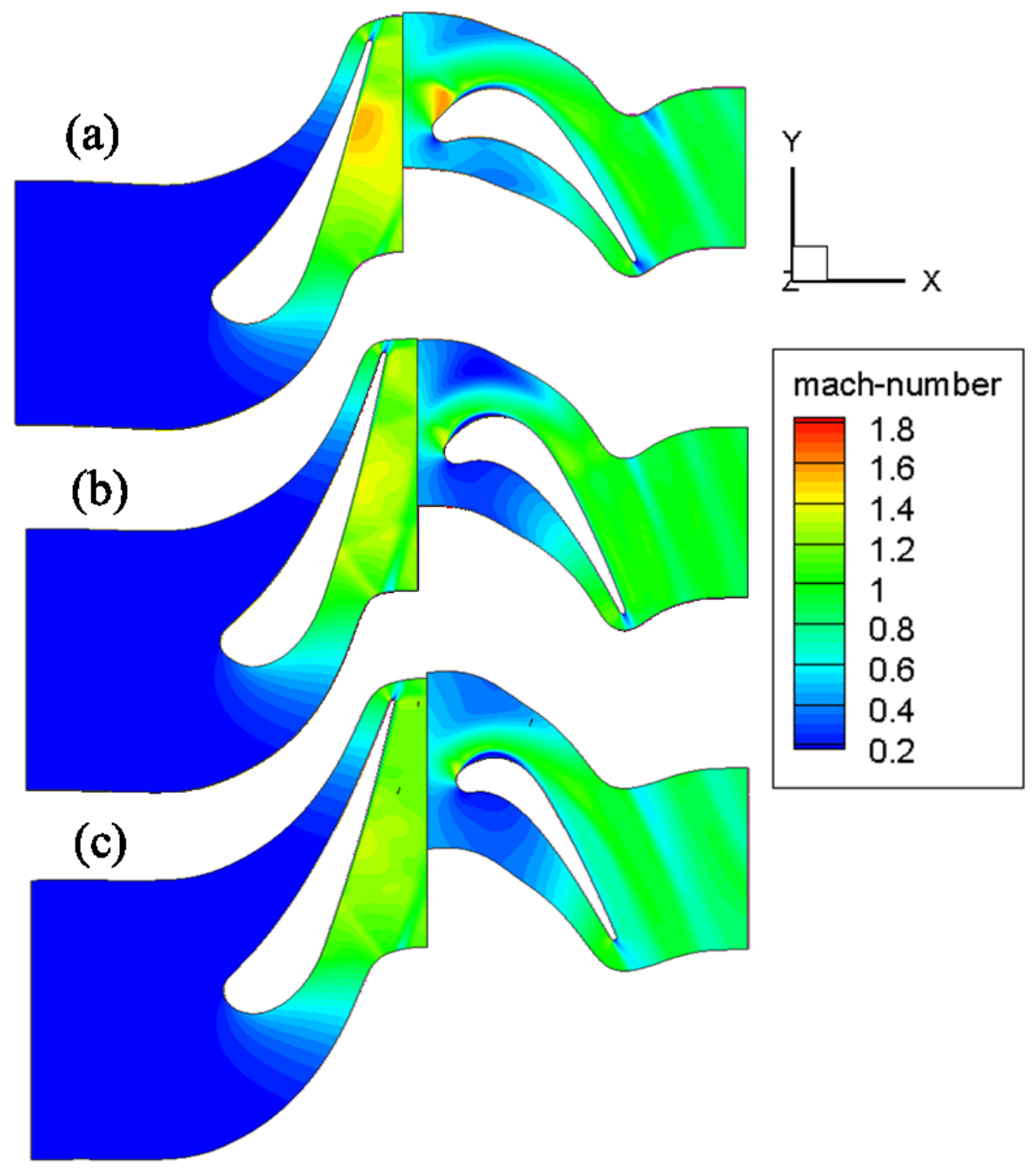

Figure 3.4: Relative and absolute Mach contours on constant radial slices, (a) hub region $30 \%$ span, (b) midspan, (c) case region $70 \%$ span 
Figure 3.5 shows the vane wake plotted on a constant radius slice at midspan of the domain with the particle locations plotted on the outlet of the vane. One can see a clear propensity for particles to move into the wake as they leave the vane domain.

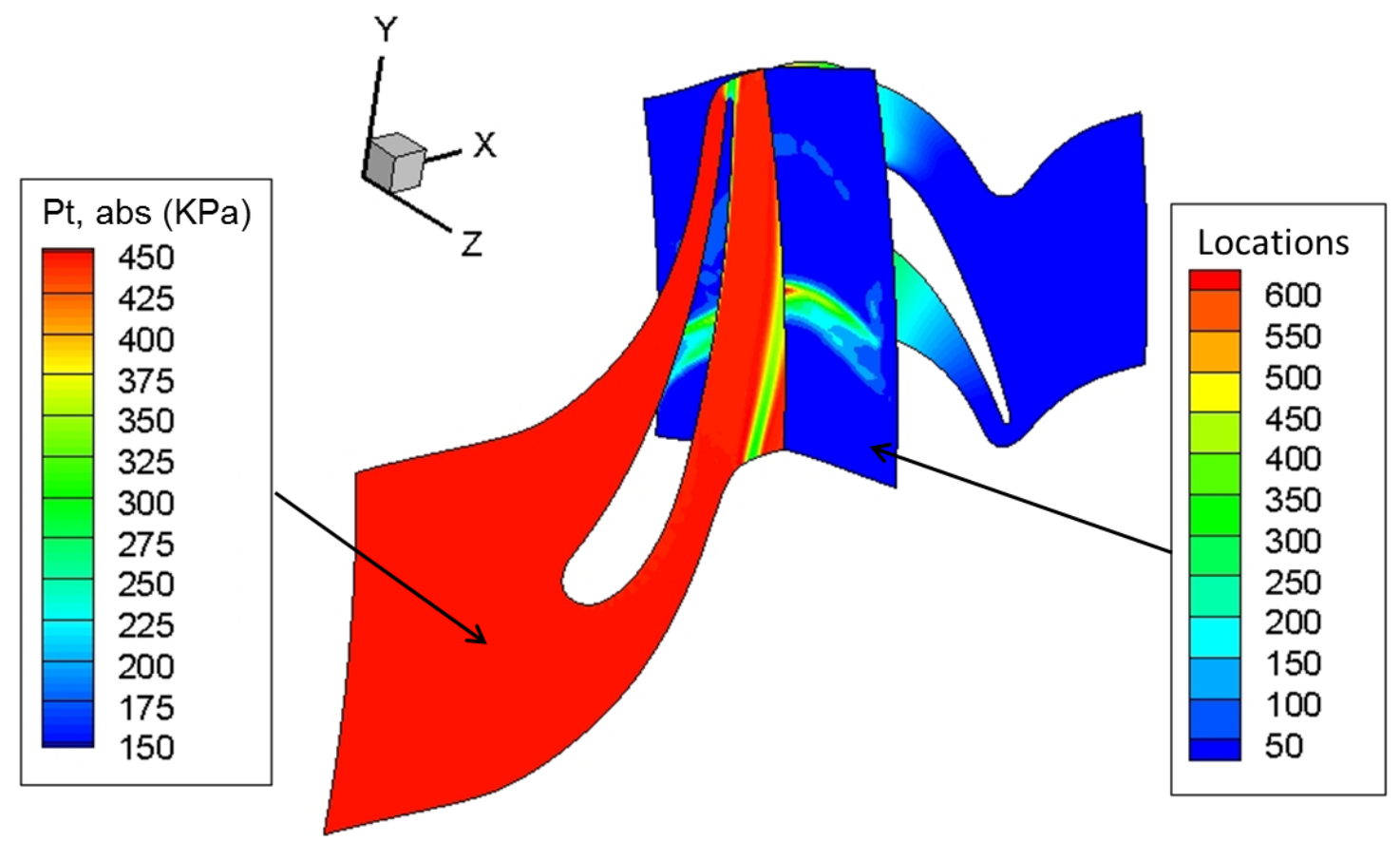

Figure 3.5: Vane wake and particle locations on mixing plane for $6 \mu \mathrm{m}$, vane outlet. Total pressure contours on $\mathbf{5 0 \%}$ span surface.

However, there are more excursions from the wake pattern for large particles due to more particles separating over the suction peak and more particles rebounding off the trailing edge as is discussed further in Section 3.1.2. Figure 3.6 shows the vane outlet particle distributions for each particle diameter demonstrating this effect. This is an important finding and will be developed more in the following discussion. 

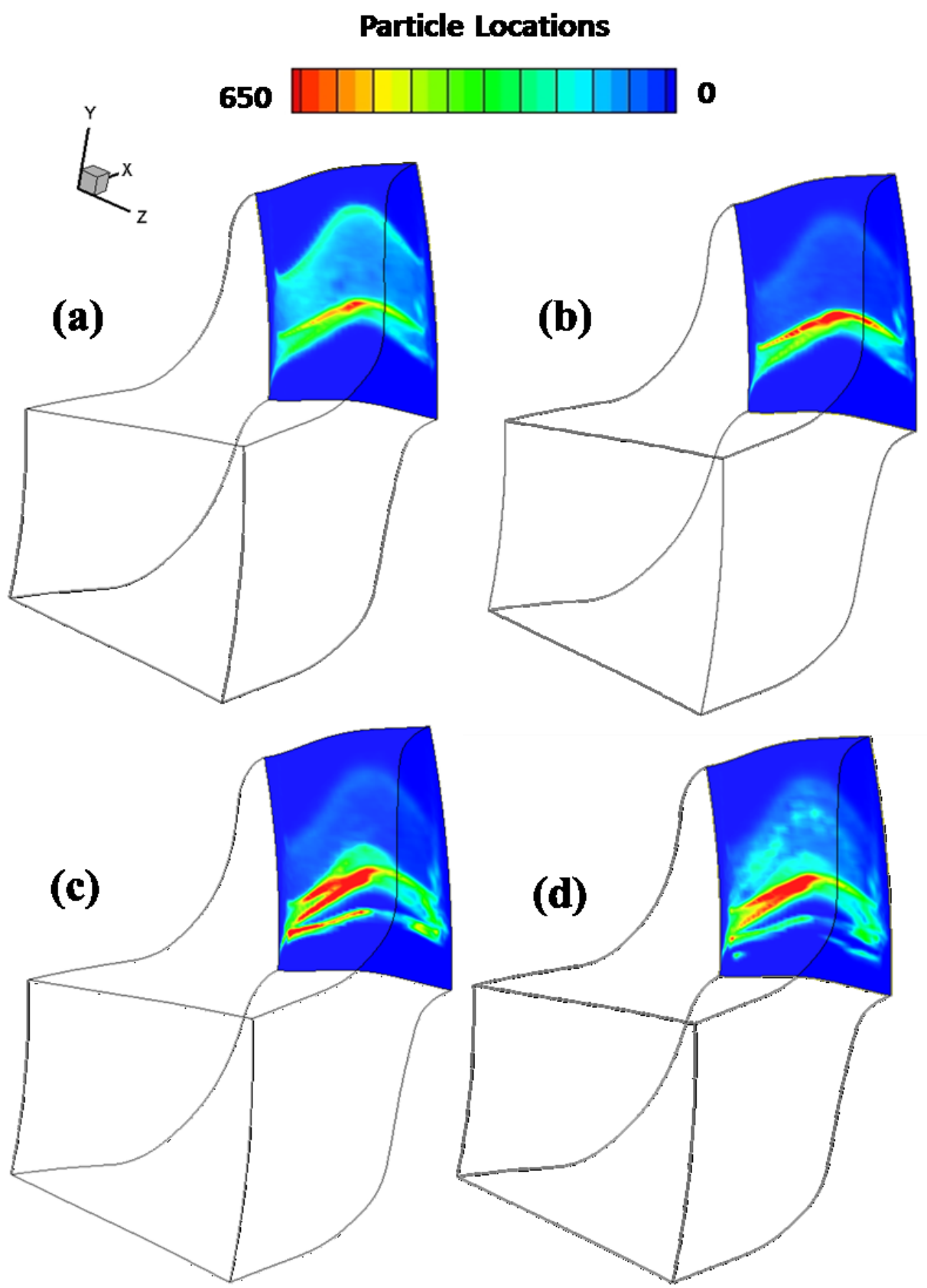

Figure 3.6: Particle locations at vane outlet for steady mixing plane solutions and particles of diameter (a) $3 \mu \mathrm{m}$, (b) $6 \mu \mathrm{m}$, (c) $10 \mu \mathrm{m}$, (d) $15 \mu \mathrm{m}$ 


\subsubsection{Steady Impacts and Deposits}

Results of steady impact and deposit distributions for all particle sizes are shown in

Figure 3.7. Note that as particle size increases the surface area of the vane and blade affected by the particle impacts and deposits shrinks. For the larger particles the impacts and deposits dominate two localized regions on the blade: the pressure surface hub behind the leading edge and the tip of the pressure surface along the entire chord. On the vane a localized region of impacts and deposits is seen towards the trailing edge across the entire span for larger particles. The hub bias of blade impacts results from particles flowing through the vane passage experiencing a larger acceleration towards the hub side due to spanwise variations in the flow (Figure 3.4). This bias creates a tendency for the higher velocity particles to impact the trailing edge of the vane pressure surface, thus entering the blade domain at a higher angle of incidence than the midspan or case side particles. The high number of vane pressure surface trailing edge impacts can be seen in the left column of Figure 3.7. As discussed before, this effect is exacerbated for larger particles with more inertia as they tend to impact the vane pressure surface more often. The combination of the larger hub-side flow acceleration, as well as separating particles rebounding off of vane pressure surface causes the tightly distributed hub side blade impacts observed in the contours. Increasing particle size also causes an increase in the number of tip impacts and deposits as well — this can be explained by an increase in centrifugal force experienced by the particles due to the increased mass of larger particles. Note that virtually no impacts or deposits were found on the suction surfaces of either the vane or the blade so they are not pictured here. 


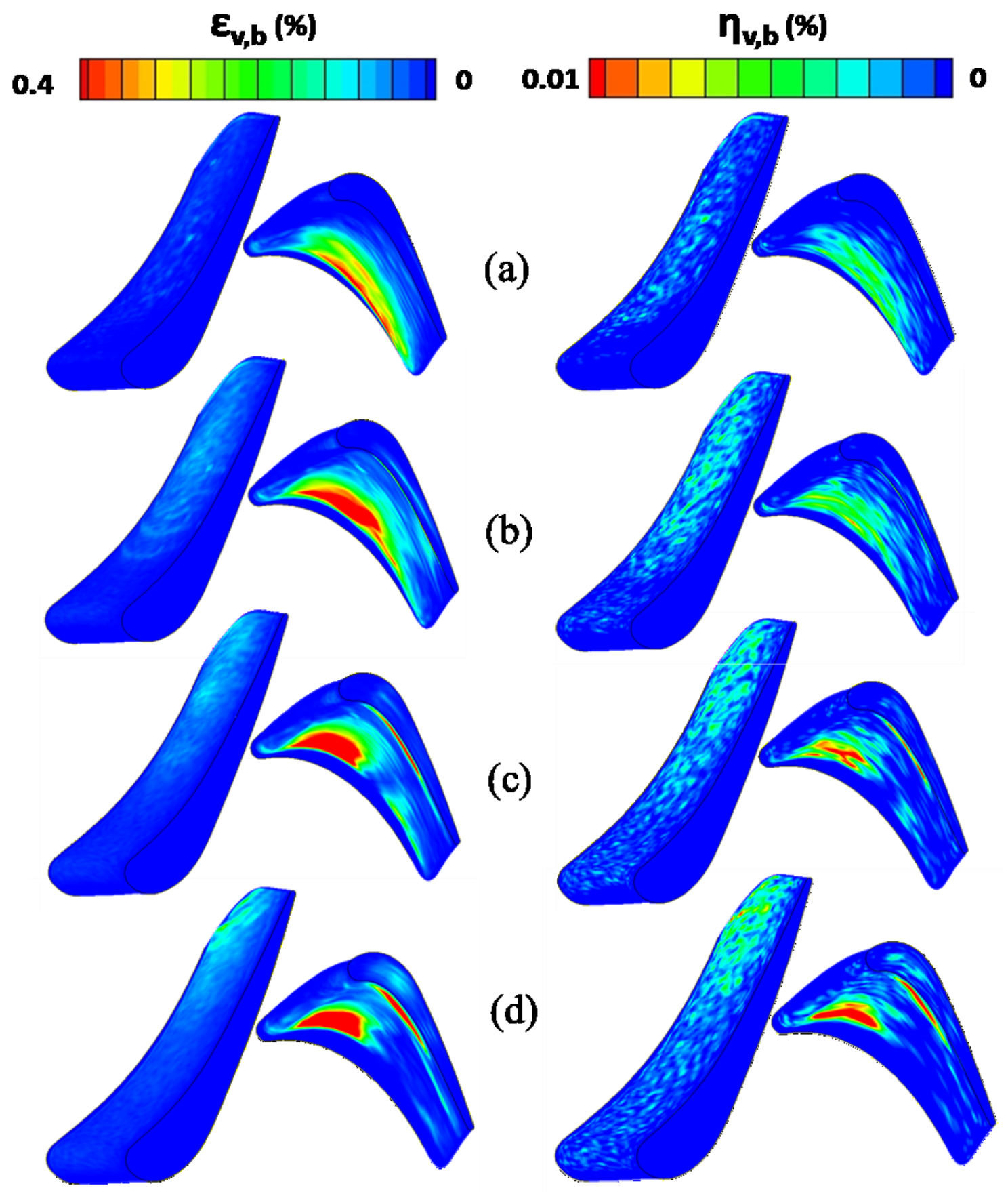

Figure 3.7: Preserved impact and deposit contours for (a) $3 \mu \mathrm{m}$, (b) $6 \mu \mathrm{m}$, (c) $10 \mu \mathrm{m}$, (d) $15 \mu \mathrm{m}$ 
Despite the impact contours showing many more impacts on the blade surface than the vane surface for all particle sizes, the right column of Figure 3.7 shows that the magnitude of blade deposits is only slightly larger than the vane deposits. This can be attributed to the lower temperature in the blade passage as work is extracted from the fluid and the expanding flow drops in pressure. Temperature is the driving force of the present deposition model so the lower temperatures give a lower probability to stick to the blade as described in Section 2.6.

\subsection{Unsteady Solution}

Flow solution results for the unsteady calculations are shown in Figure 3.8. Absolute and relative total pressure contours are shown on midspan slices through the vane and blade domains, respectively. The light green regions are areas of viscous loss from the vane and blade surfaces, i.e. wakes. The steady solution (Figure 3.5) showed a propensity for particles to follow the vane wake out of the vane domain. Therefore, knowing the location of the vane wakes is important in understanding the particle movement in the unsteady case. A very important phenomenon to notice is the unsteady motion of the discrete vane wakes as they migrate through the blade passage. Observe the wake from vane 1 in Figure 3.8: at time (i) the vane 1 wake impinges on the leading edge of the blade 2, at time (ii) the wake moves to the pressure surface of blade 2 , at time (iii) the wake starts to become chopped by blade 1, at time (iv) the wake is fully chopped by blade 1 and no longer impinges on blade 2, at time (v) the wake begins to impinge on the pressure surface of blade 1 . 


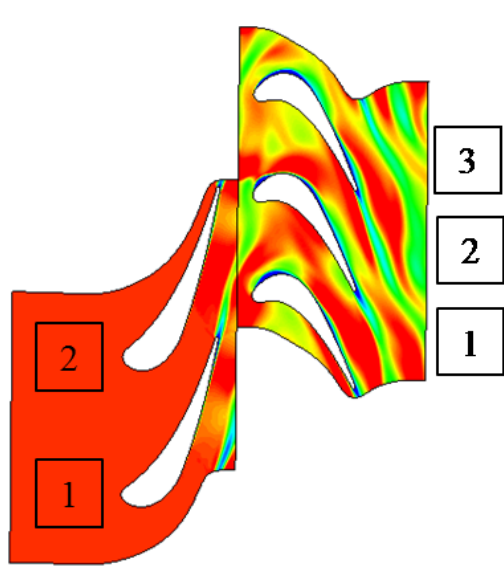

(i)

\begin{tabular}{|r|}
\hline $\mathrm{Pt}[\mathrm{kPa}]$ \\
445 \\
425 \\
\\
405 \\
385 \\
\hline 365 \\
345 \\
325 \\
305 \\
285 \\
265 \\
265 \\
245 \\
225 \\
\hline
\end{tabular}

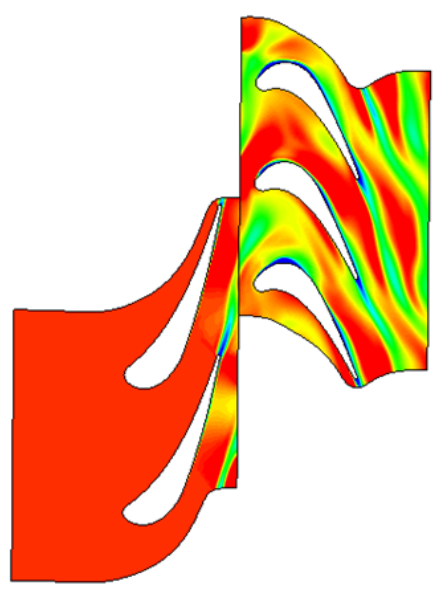

(ii)

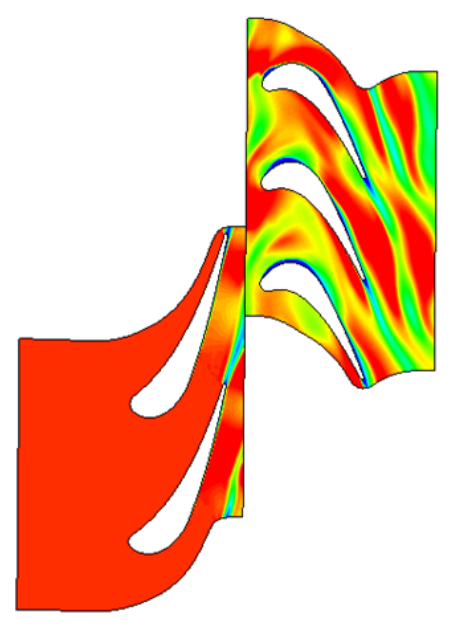

(iii)
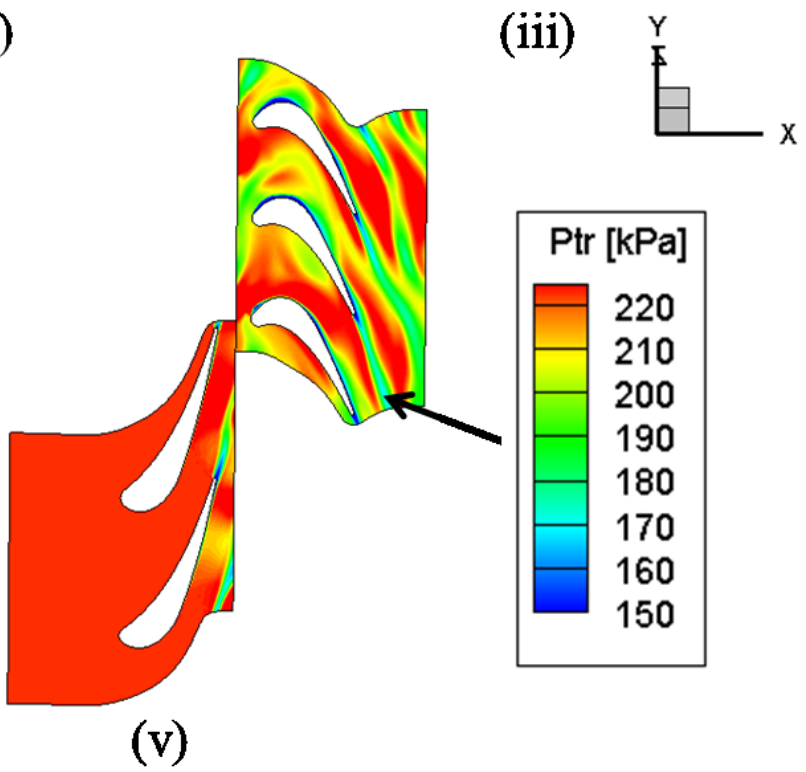

(iv)

(v)

Figure 3.8: Midspan absolute and relative total pressure contours at (i) $t=\mathbf{t}_{\mathbf{0}}$, (ii)

$$
t=4 \Delta t+t_{0}, \text { (iii) } t=8 \Delta t+t_{0}, \text { (iv) } t=12 \Delta t+t_{0}, \text { (v) } t=16 \Delta t+t_{0}
$$

Because the vane wakes are characterized by a deficit in total pressure as compared to the free stream, they are also characterized by a velocity deficit in the absolute frame. When the vane wake velocity is converted to the rotor relative frame by subtracting the 
rotational velocity of the rotor, the resulting vector is biased away from the pressure surface and towards the suction surface relative to the non-wake passage flow. Figure 3.9 demonstrates this argument graphically with example velocity triangles in the free stream and the wake fluid, respectively.

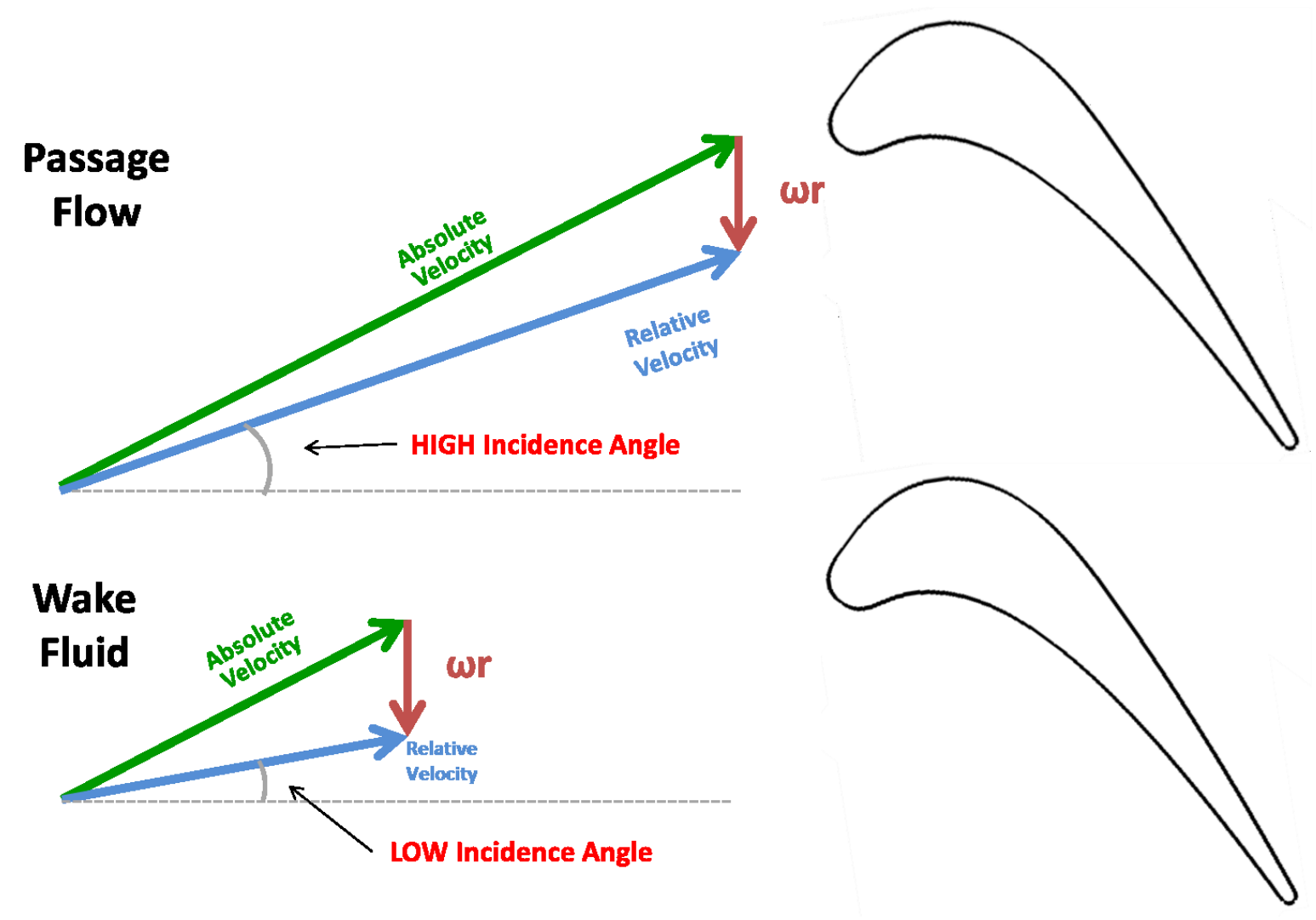

Figure 3.9: Velocity triangle schematics for free stream and wake fluid with averaged velocity values

Note that the lower incidence angle of the wake fluid, due to the retarded absolute velocity, will cause particles to avoid contact with the blade, resulting in fewer impacts on the pressure surface of the blade. 
This wake velocity effect is evident in Figure 3.8 as the vane wake is observed to avoid direct contact with much of the blade's pressure surfaces. It should be noted that due to the mixing plane interface used in the steady solutions, total pressure variations, which would include wakes, are averaged out and no discrete wake enters the blade domain. Therefore there is no velocity deficit with which to influence particles away from the pressure surface in the steady case. This is a key finding of the study and the discussion is continued in later parts of Section 3.2.

\subsubsection{Unsteady Particle Motion, 3 micron particle size}

Figure 3.10 shows particle locations and midspan total pressure contours (absolute and relative in the vane and blade domains, respectively) for the blade unsteady 3 micron case at 7 different instances of time. Note that the total pressure contour pictures are taken using larger time steps than the plots in Figure 3.8 in order to demonstrate specific features. The figure demonstrate the particles following the regions of total pressure deficit as they move through the rotor passage, eventually congregating in the blade wakes before leaving the domain. Specifically, one can see the small particles' tendency, due to their small size and inertia, to follow the wake pattern through the passage. Figure 3.10 shows wakes and particles moving through the blade passage in an ordered fashion, largely avoiding the pressure surface of the blades when compared with the steady particle tracks of Figure 3.3. The vane wakes' tendency to avoid the blade pressure surface is the key difference between the particle trajectories of the steady and unsteady calculations. 
There is also a propensity of particles to impinge on the blade near the hub side as shown in Figure 3.11, with a localized impact region at about 25\% span from the hub. This bias has been attributed to span wise variations in acceleration through the vane passage and is discussed in Figure 3.4. In order to characterize particle motion at another span wise location on the blade surface, particles were plotted in a small radial slice (from $97 \%$ to $100 \%$ span) encompassing the tip gap region of the blade and shown in Figure 3.12. The particle trajectories in this region are much different than at the midspan location. In general we see that particles do pass through the tip gap region and that there is significant out of plane motion of particles. Out of plane motion is evidenced by the number of particles in the field of view being reduced from times (iv) to (v) and (vi). This general tendency towards out of plane particle motion may be due to interactions with the case wall and the tip vortex of the blades. Note also that the case wall is not a constant radius, adding significant complexity to the flow field in that region. 

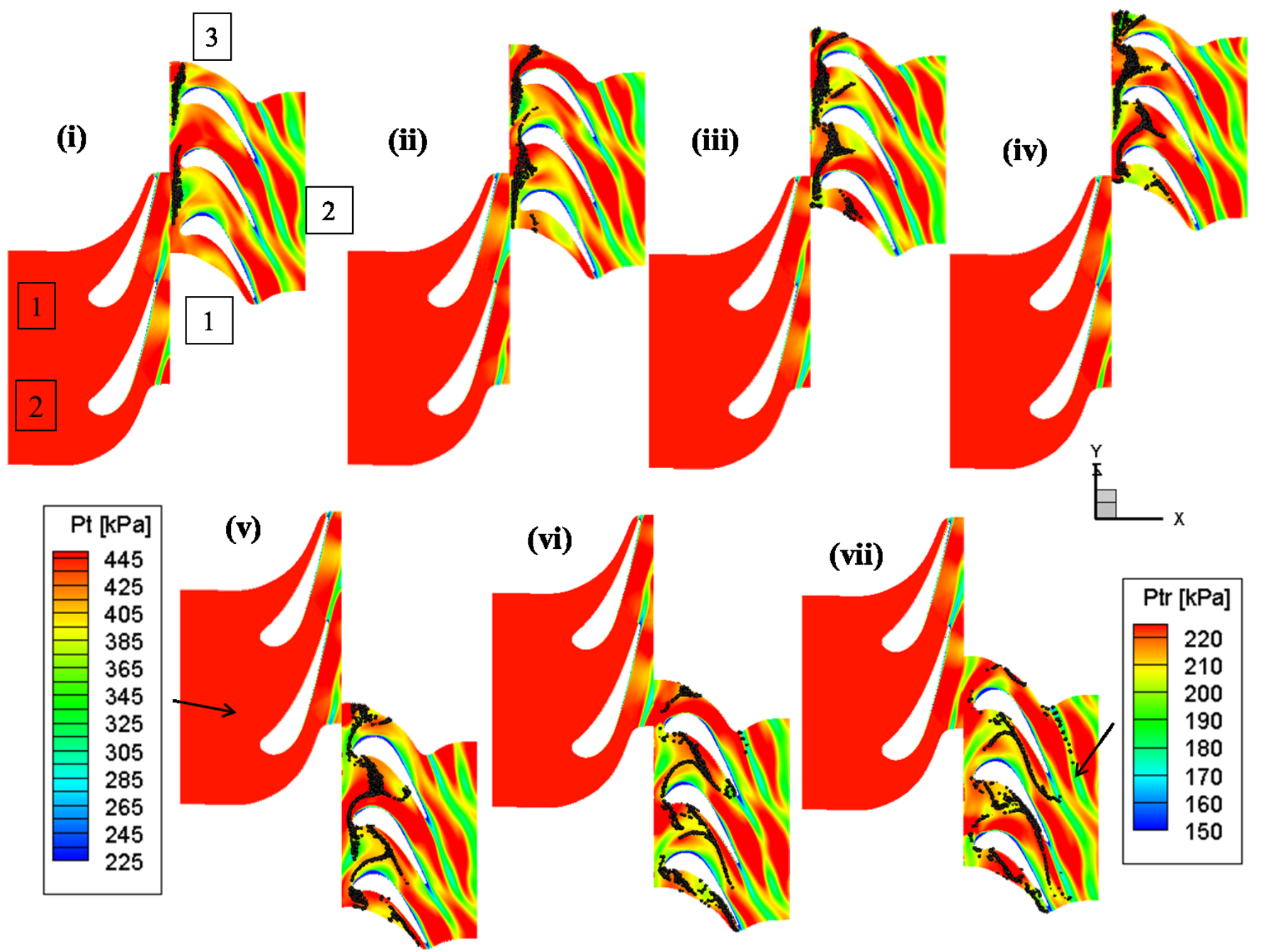

Figure 3.10: Midspan, unsteady $3 \mu \mathrm{m}$ particle locations and wakes at (i) $t=\Delta t+t_{0}$, (ii) $t=6 \Delta t+t_{0}$, (iii) $t=11 \Delta t+t_{0}$, (iv) $t$ $=16 \Delta t+t_{0},(v) t=21 \Delta t+t_{0}$, (vi) $t=26 \Delta t+t_{0}$, (vii) $t=31 \Delta t+t_{0}$ 


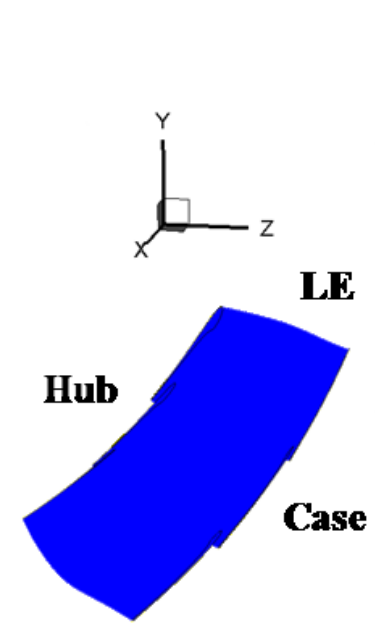

(i)

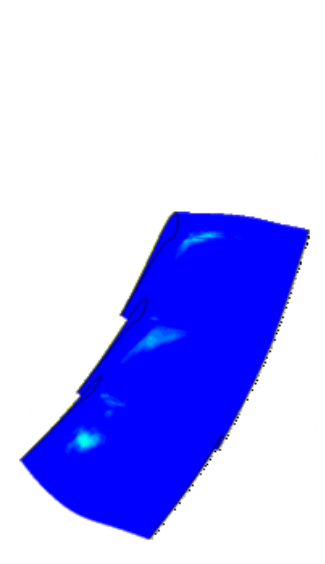

(ii)

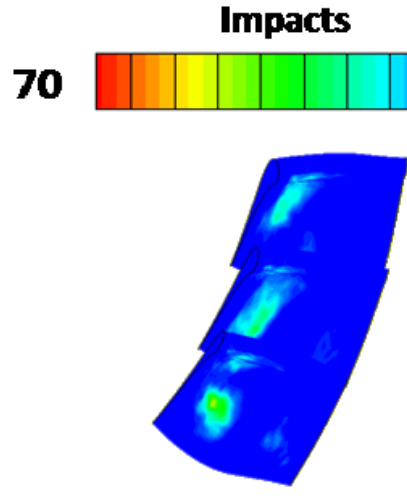

(iii)
5

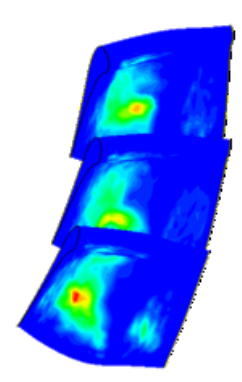

(iv)

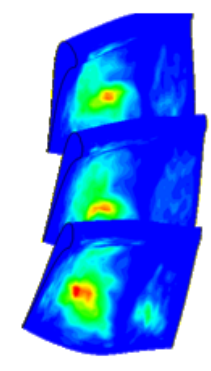

(v)

2

Figure 3.11: Unsteady $3 \mu \mathrm{m}$ blade PS impacts at (i) $t=t_{0}$, (ii) $t=16 \Delta t+t_{0}$, (iii) $t=32 \Delta t+t_{0}$ (iv) $t=48 \Delta t+t_{0}$, (v) $t=60 \Delta t+t_{0}$ 


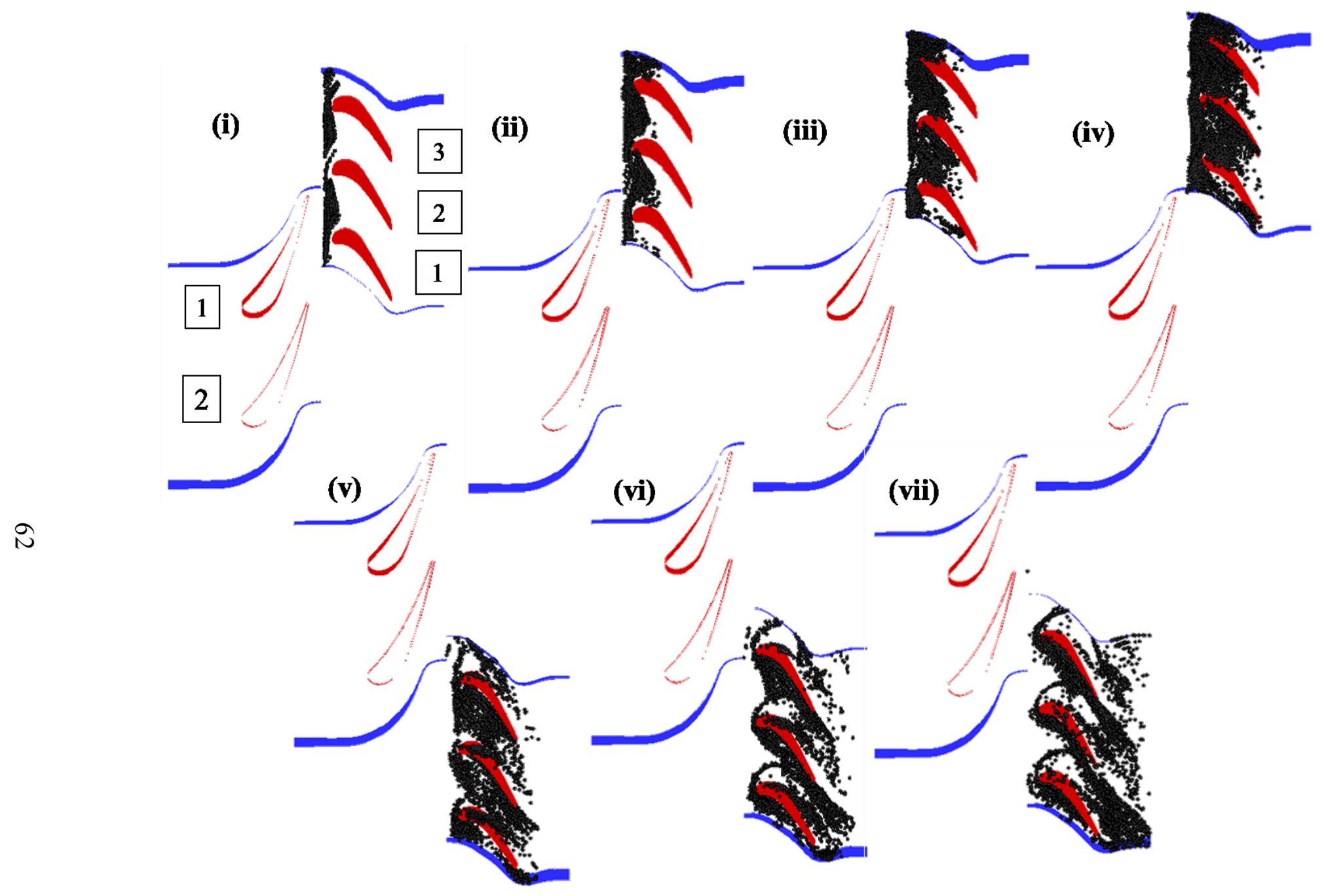

Figure 3.12: Tip gap (97-100\% span), unsteady $3 \mu \mathrm{m}$ particle locations and wakes at (i) $t=\Delta t+t_{0}$, (ii) $t=6 \Delta t+t_{0}$, (iii) $t=11 \Delta t+t_{0}$, (iv) $t=16 \Delta t+t_{0}$, (v) $t=21 \Delta t+t_{0}$, (vi) $t=26 \Delta t+t_{0}$, (vii) $t=31 \Delta t+t_{0}$ 


\subsubsection{Unsteady Impacts and Deposits, 3 micron}

Figure 3.13 shows impact and deposit contours for the 3 micron unsteady and steady cases. Note that the three blade surfaces of the unsteady calculation have been summed together and show the total impact contours over the three blades for one vane passing period. Due to the periodicity of the steady solution where particles leave and reenter periodic boundaries, summing the three blades was deemed to be the closest comparison. The impact patterns are similar for the unsteady and steady cases with a localized region towards the hub, however, the steady case clearly has a much higher number of impacts and deposits. Also the locus of impacts and deposits seems to be shifted farther back in the chordwise direction in the unsteady than the steady case. Reasons for this difference will be explained in sections 3.2.5 and 3.2.6. 


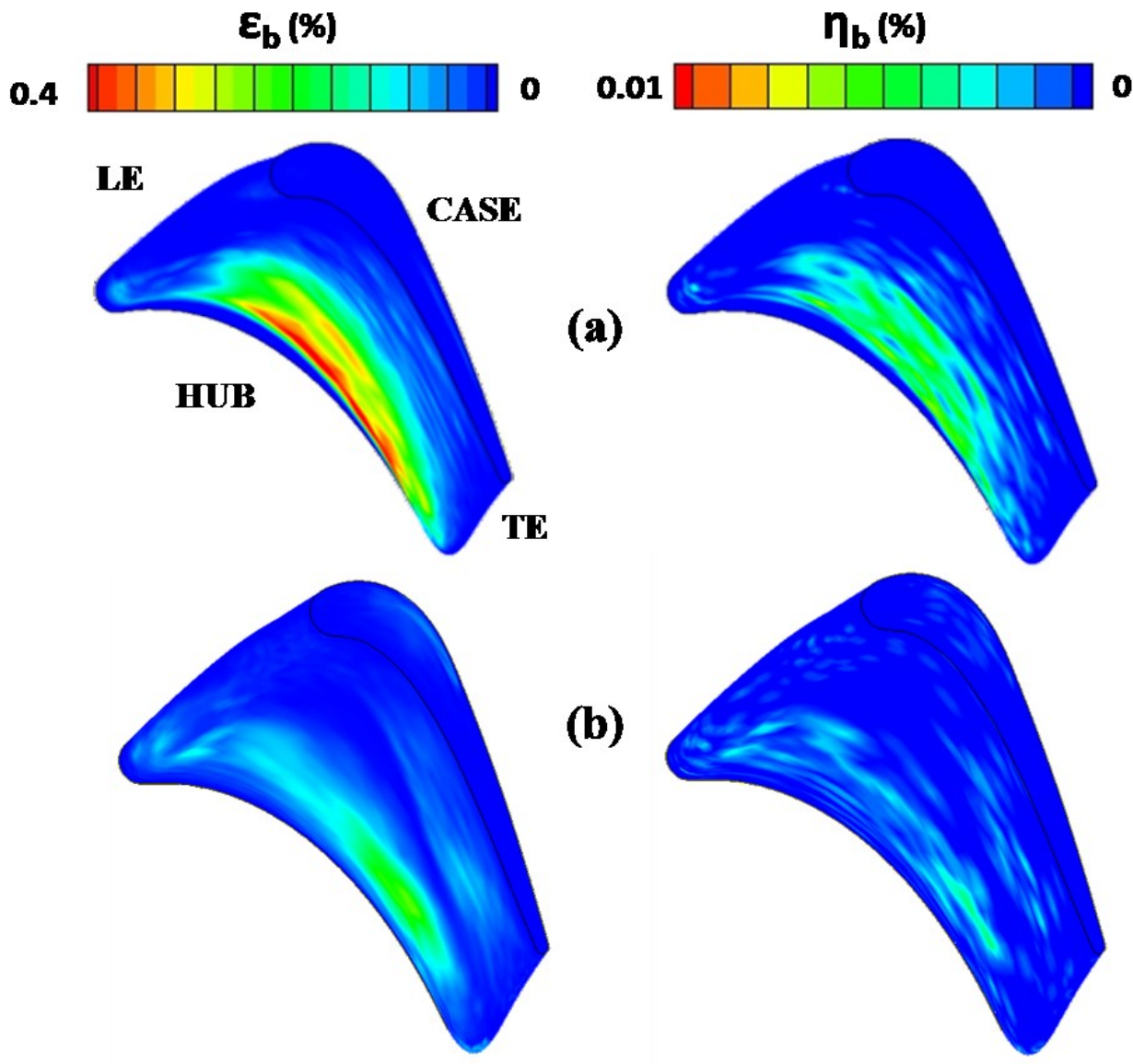

Figure 3.13: Aggregate impact and capture efficiency contours for $3 \mu \mathrm{m}$ particles (a) steady, (b) unsteady

It should also be noted that the steady solution has virtually no impacts on the suction surface while the unsteady solution had a noticeable amount ( $1 \%$ of total). This observation corroborates the claim that the preserved vane wake motion in the unsteady case caused a suction side bias of particle motion whereas the steady case's averaged wake (in the mixing plane) did not capture this effect. The deposit contours are also similar in location but again the steady case has a much higher magnitude of deposits. These differences are quantified in Section 3.3. 


\subsubsection{Unsteady Particle Motion, 6 micron}

Figure 3.14 shows particle locations and midspan total pressure contours (absolute and relative in the vane and blade domains, respectively) for the unsteady 6 micron case at 7 different instances of time. In general, particles are shown to follow the wake trajectory at early time steps. At later time steps the particle trajectories deviate somewhat from the wake locations when compared with the 3 micron case in Figure 3.10. Some particles rebound off of the pressure surface as shown in time (vi) and (vii) of Figure 3.14 and actually strike the suction surface. Examples of those rebounding are circled in purple. The 6 micron case did yield many more impacts and deposits on the suction surface than the 3 micron case due to the higher number of ricochets experiences $(9 \%$ and $1 \%$ of total, respectively). Note that rebounds from blade pressure surface to suction surface do not occur in the steady cases and there are virtually no suction surface impacts. Reasons for this difference between unsteady and steady calculations are explained in Sections 3.2.5 and 3.2.6.

Figure 3.15 shows particles plotted in a small slice at the tip gap region of the blade. The particle trajectories in this region are less well organized than at the midspan. In general there is significantly more out of plane motion of particles than in the 3 micron case. Specifically, one can see the total number of particles in the field of view being reduced significantly from times (iv) to (v). This motion may be due to significant leading edge impacts that ricochet back into the vane domain slightly, changing particles' spanwise locations. At times (vi) and (vii) particles can be seen grouping up into more ordered trajectories as they move through the passage. 

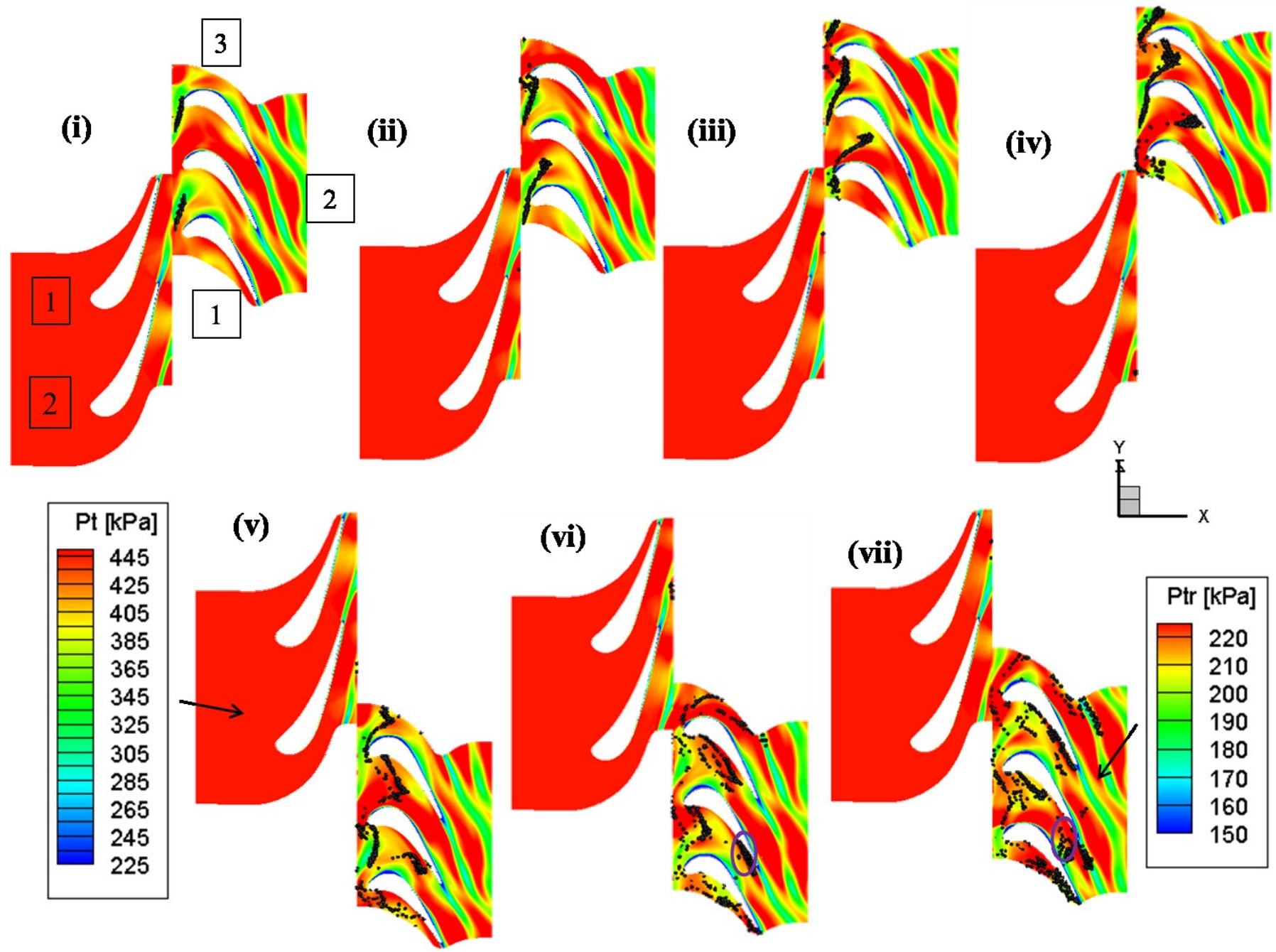

Figure 3.14: Midspan, unsteady $6 \mu \mathrm{m}$ particle locations and wakes at (i) $t=\Delta t+t_{0}$, (ii) $t=6 \Delta t+t_{0}$, (iii) $t=11 \Delta t+t_{0,}$ (iv) $t=16 \Delta t+t_{0}$, (v) $t=21 \Delta t+t_{0}$, (vi) $t=26 \Delta t+t_{0}$, (vii) $t=31 \Delta t+t_{0}$ 


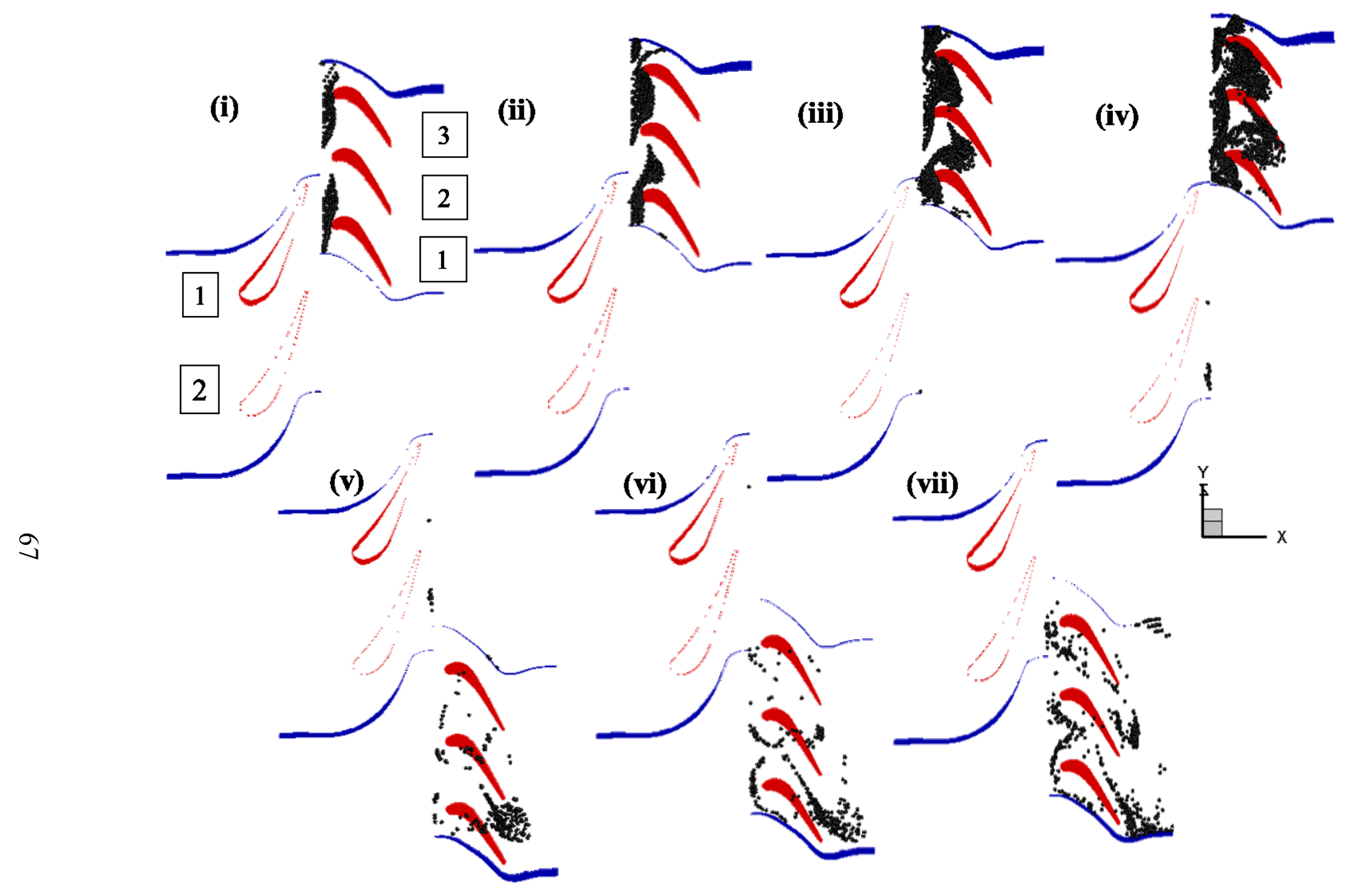

Figure 3.15: Tip gap (97-100\% span), unsteady $6 \mu \mathrm{m}$ particle locations at (i) $t=\Delta t+t_{0}$, (ii) $t=6 \Delta t+t_{0}$, (iii) $t=11 \Delta t+t_{0}$, (iv) $t=16 \Delta t+t_{0}$, (v) $t=21 \Delta t+t_{0}$, (vi) $t=26 \Delta t+t_{0}$, (vii) $t=31 \Delta t+t_{0}$ 


\subsubsection{Unsteady Impacts and Deposits, 6 micron}

Figure 3.16 shows impact and deposit contours for the 6 micron unsteady and steady cases. Note that the three blade surfaces of the unsteady calculation have been summed together and show the total impact contours over the three blades for one vane passing period like in the previous case. The impact patterns are quite different for the unsteady and steady cases at this particle size. Generally, the distribution favors the hub side but the locus of impacts is shifted significantly farther back in the chordwise direction in the unsteady than the steady case. Interestingly, the locus for the 6 micron unsteady is very similar in location to the 3 micron unsteady shown in Figure 3.13. Also the deposit distribution for the unsteady case is much more diffuse and distributed across the span of the blade. Also, Figure 3.17 it is shown that there are significant suction side impacts and deposits at the 6 micron size. These impacts and deposits do not occur in the steady version. Reasons for these differences will be discussed in Sections 3.2.5 and 3.2.6. 

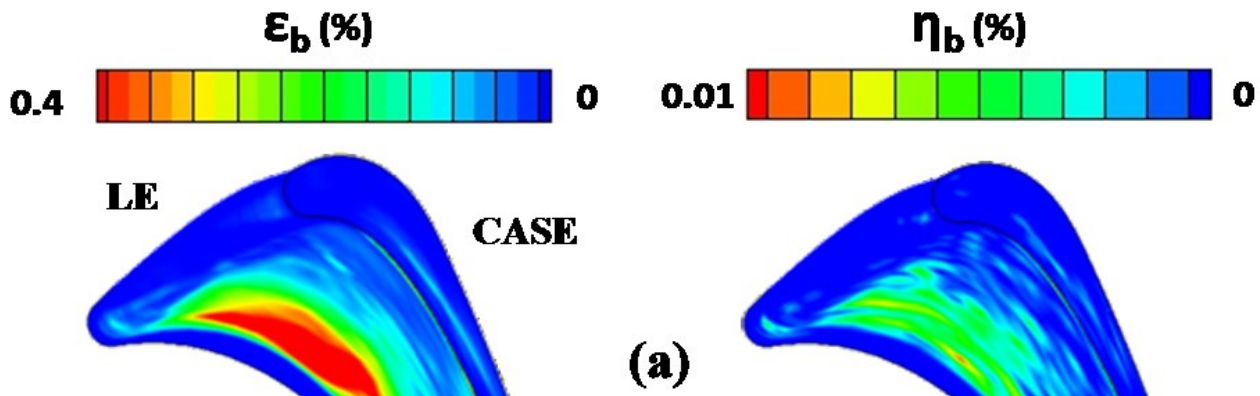

(a)
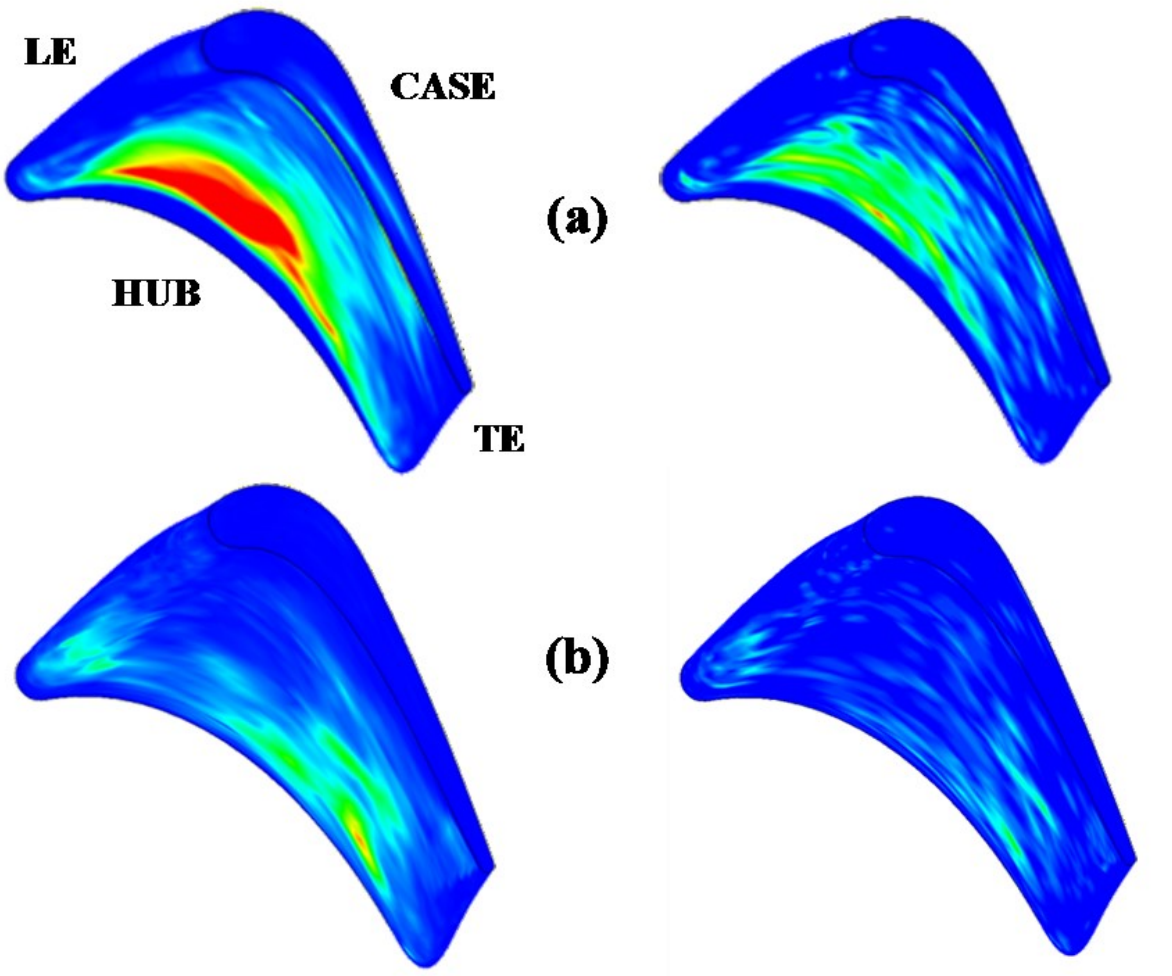

Figure 3.16: Aggregate PS impact and capture efficiency contours for $6 \mu \mathrm{m}$ particles (a) steady, (b) unsteady
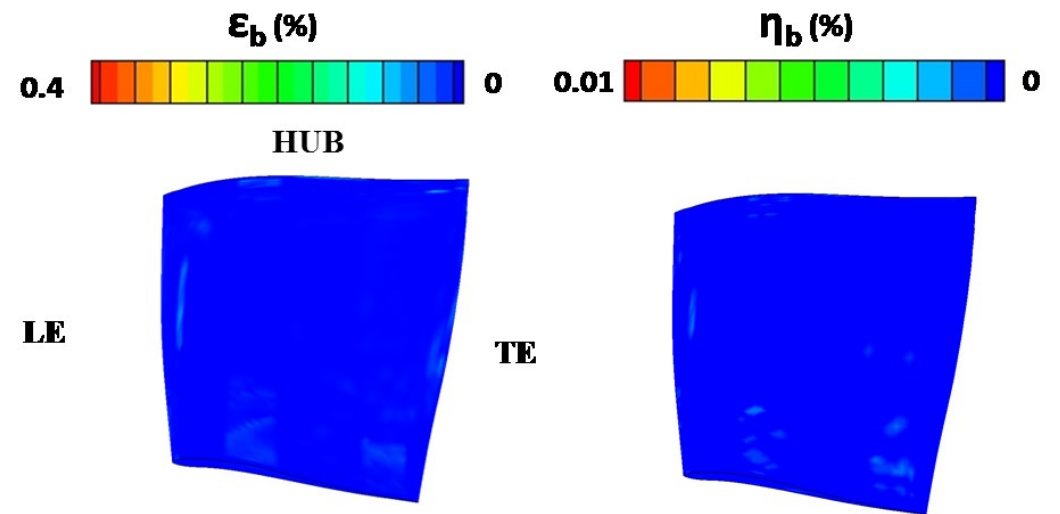

(b)

CASE

Figure 3.17: Aggregate SS impact and capture efficiency contours, $6 \mu \mathrm{m}$ unsteady 


\subsubsection{Unsteady Particle Motion, 10 micron}

Figure 3.18 shows particle locations and midspan total pressure contours (absolute and relative in the vane and blade domains, respectively) for the blade unsteady 10 micron case at 7 different instances of time. In general, particles deviate from the wake trajectory quite rapidly due to their larger size and inertia associated with their larger Stokes number (30 vs. 3 and 11 for 10, 3 and 6 micron, respectively). At later time steps the particle trajectories deviate even more and form more disorganized trajectories when compared with the steady 10 micron case in Figure 3.10. Many particles rebound off of the pressure surface as shown in time (vi) and (vii) of Figure 3.14 and strike the suction surface. Examples of those rebounding are circled in grey. The 10 micron case did yield many more impacts on the suction surface than the 6 micron case, $40 \%$ and $9 \%$ of total, respectively, due to the higher number of pressure surface ricochets experienced. Note that rebounds from blade pressure surface to suction surface do not occur in the steady cases and there are virtually no suction surface impacts in those cases. 


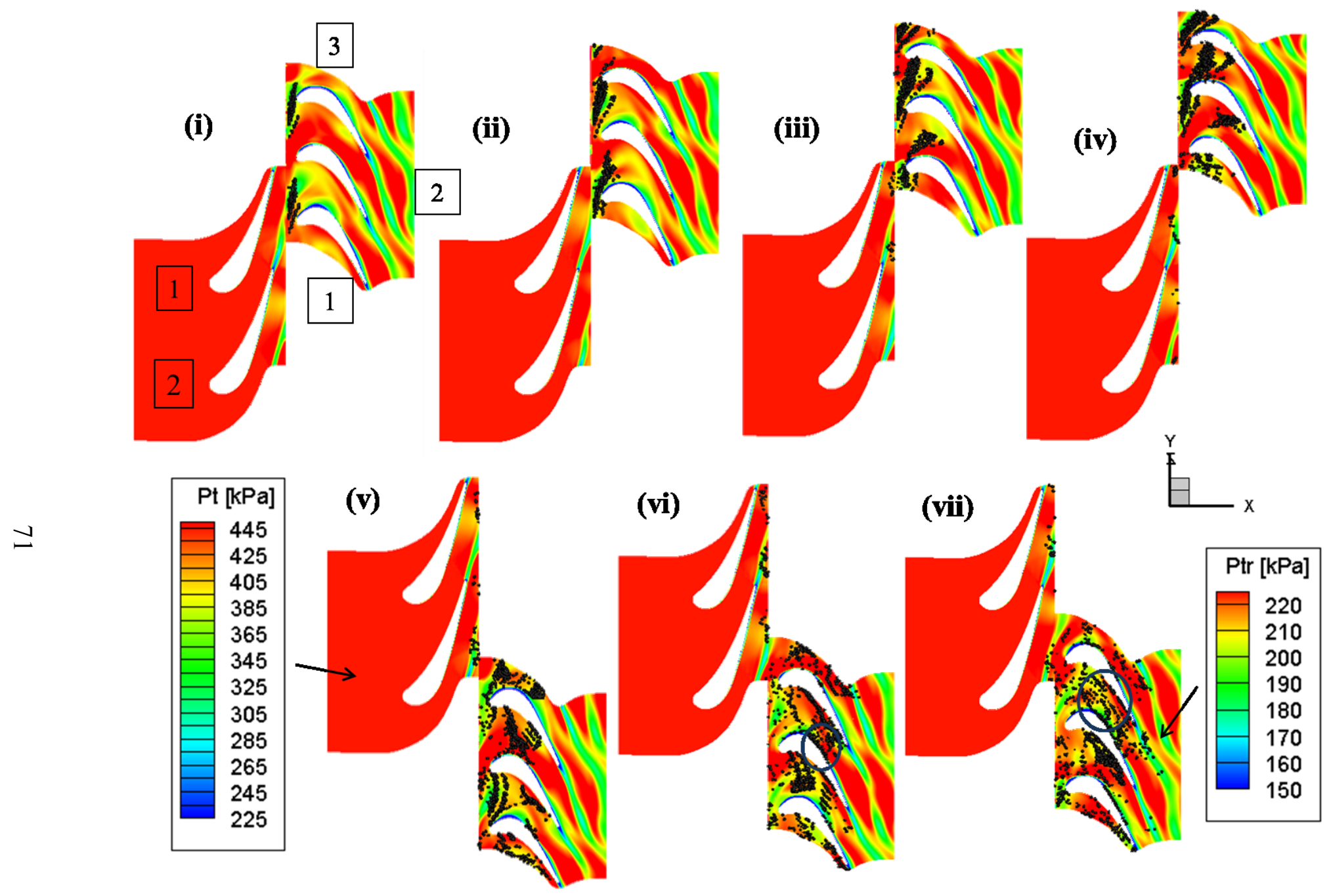

Figure 3.18: Midspan, unsteady $10 \mu \mathrm{m}$ particle locations and wakes at (i) $t=\Delta t+t_{0}$, (ii) $t=6 \Delta t+t_{0}$, (iii) $\mathrm{t}=11 \Delta \mathrm{t}+\mathrm{t}_{0}$, (iv) $\mathrm{t}=16 \Delta \mathrm{t}+\mathrm{t}_{0}$, (v) $\mathrm{t}=21 \Delta \mathrm{t}+\mathrm{t}_{0}$, (vi) $\mathrm{t}=26 \Delta \mathrm{t}+\mathrm{t}_{0}$, (vii) $\mathrm{t}=31 \Delta \mathrm{t}+\mathrm{t}_{0}$ 
The lack of suction side impacts in all of the steady solutions could be due to two important differences relative to the unsteady solutions: the locations of initial pressure surface impacts shift back from $30 \%$ to $70 \% \mathrm{WD}$ in the unsteady results and time variations in the passage Mach number are evidenced. The first difference will be discussed later in Section 3.2.6. Figure 3.19 shows midspan Mach contours for the steady flow solution and at different instances of time for the unsteady solution. Interestingly, it can be seen that the steady solution has a higher constant Mach number through the blade passage and that due to the blade unsteadiness, regions of low Mach number (circled in purple) can stretch as far as $70-80 \%$ of blade axial chord at different instances in time. 


\begin{tabular}{|c|}
\hline mach-number \\
1.8 \\
1.6 \\
1.4 \\
1.2 \\
1 \\
0.8 \\
0.6 \\
0.4 \\
0.2 \\
\hline
\end{tabular}

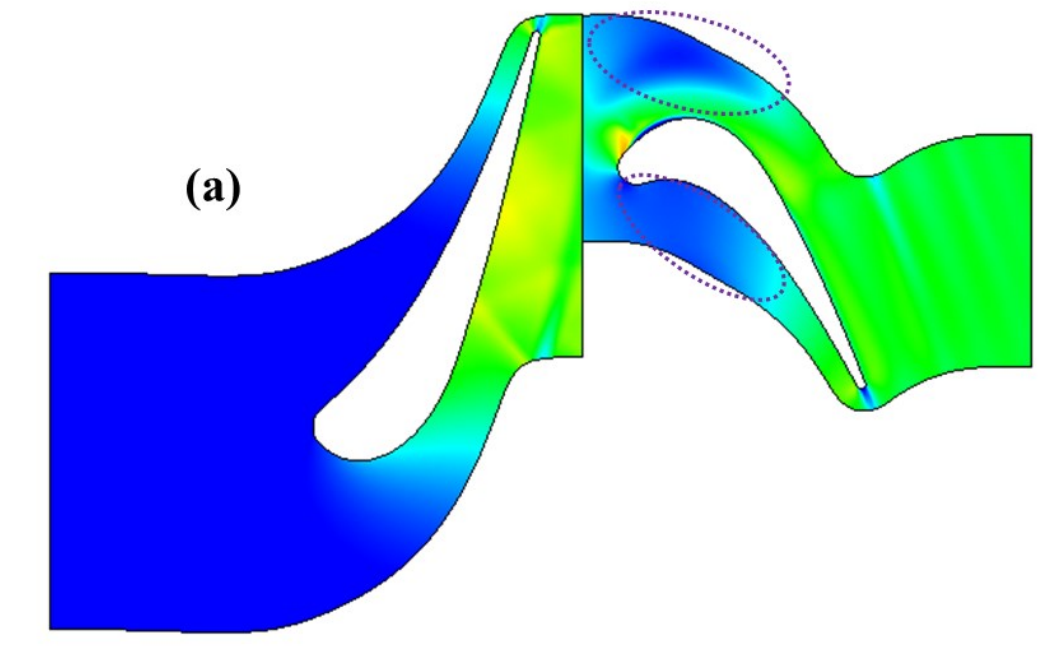

w
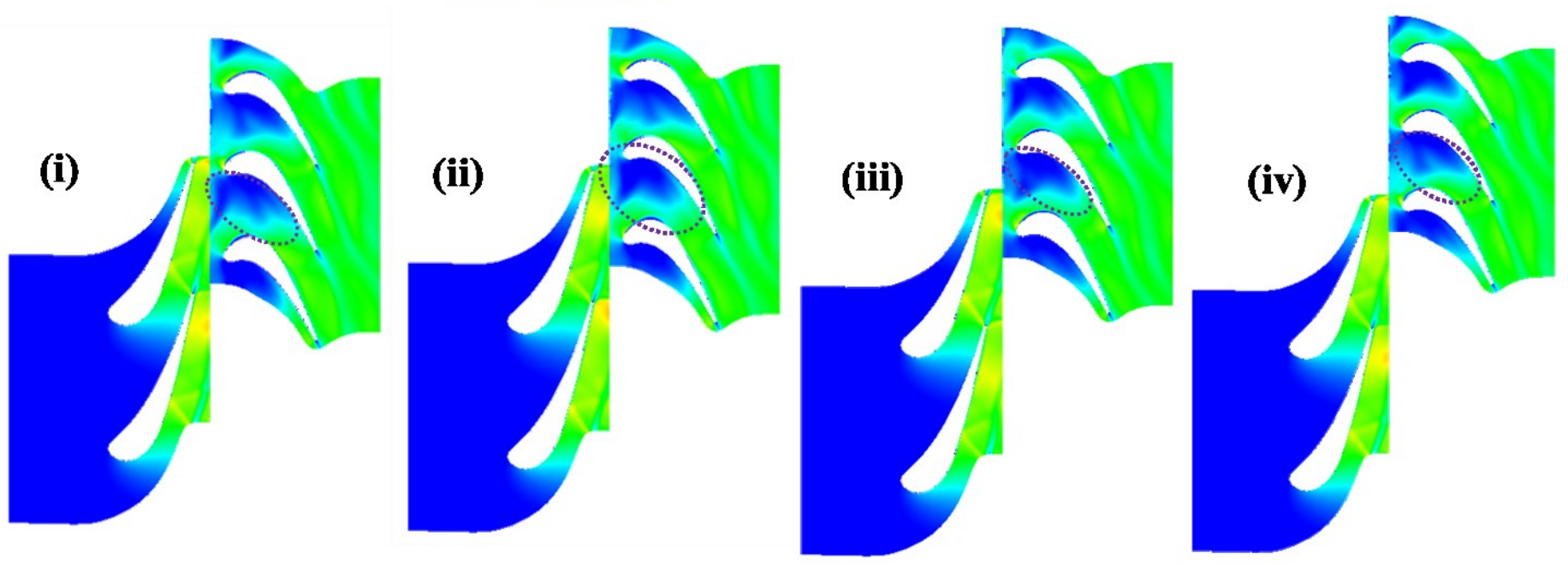

Figure 3.19: Midspan Mach contours (a) steady, and unsteady at (i) $t=\Delta t+t_{0}$, (ii) $t=6 \Delta t+t_{0}$, (iii) $t=11 \Delta t+t_{0}$, (iv) $\mathrm{t}=16 \Delta \mathrm{t}+\mathrm{t}_{0}$ 
Particles that rebound off of the pressure surface during times of low passage Mach number will experience less drag and be more likely to traverse the passage to the suction surface. In contrast, rebounding particles in the steady simulations will experience more drag in the constant Mach number flow and be more readily re-entrained into the flow, avoiding an impact with the suction surface of the neighboring blade. Figure 3.20 shows particle tracks for the steady 10 micron case — particles impact the pressure surface, pass through the periodic boundary and are re-entrained quickly before they can impact the suction surface.
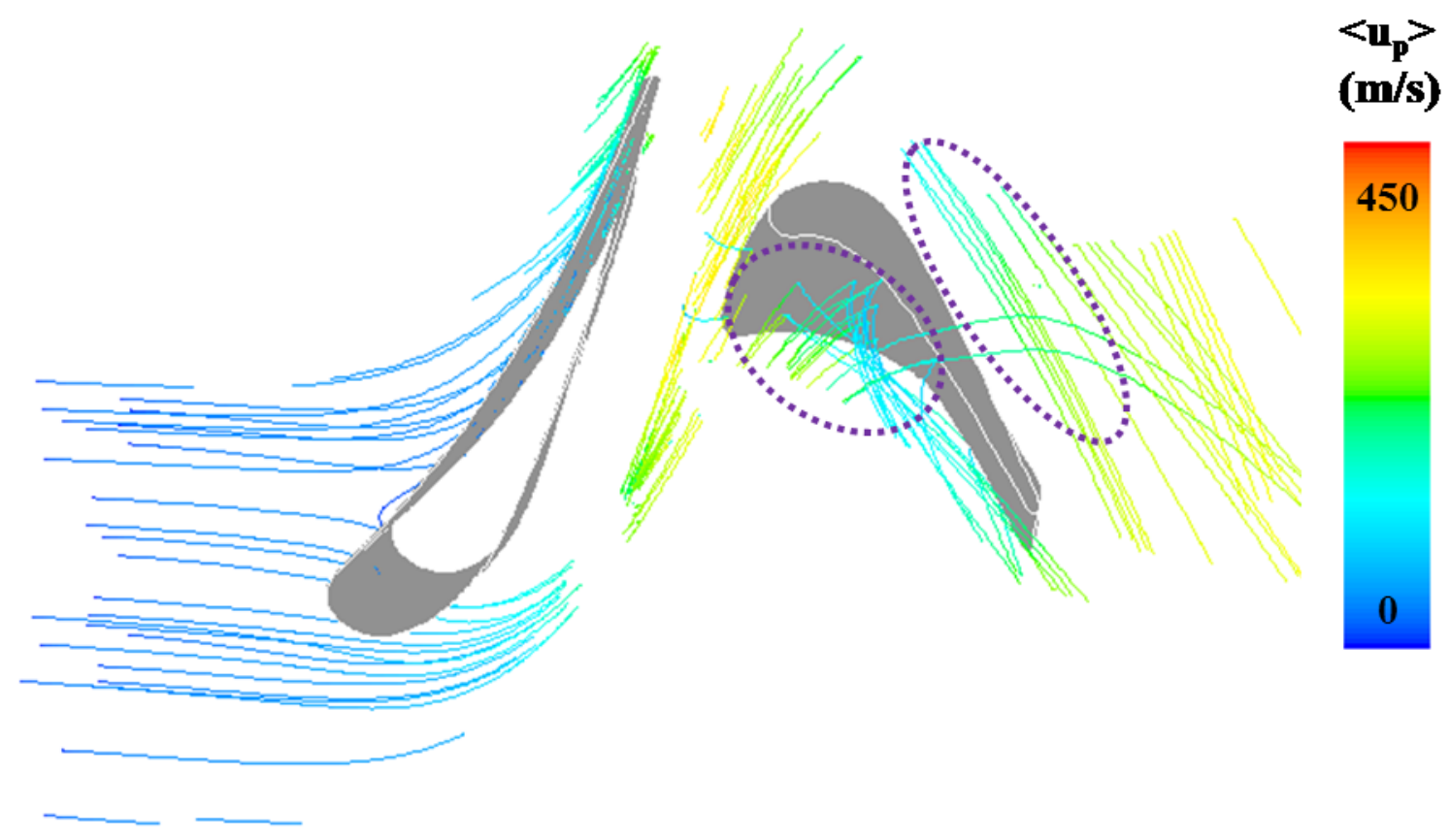

Figure 3.20: Steady, 10 micron particle pathlines colored by particle velocity magnitude

The suction surface rebounds that occur in the unsteady cases account for a significant number of impacts, nearly as many as the pressure surface. These figures will be analyzed quantitatively in section 3.3. 
Figure 3.21 shows particles plotted in a small slice at the tip gap region of the blade. The particle trajectories in this region are again less organized than at the midspan. In general there is significantly more out of plane motion of particles than in the 3 or 6 micron case. Specifically, one can see the total number of particles in the field of view being reduced significantly from times (iv) to (v). This motion may be due to significant leading edge impacts that ricochet back into the vane domain slightly, changing particles' spanwise locations. 


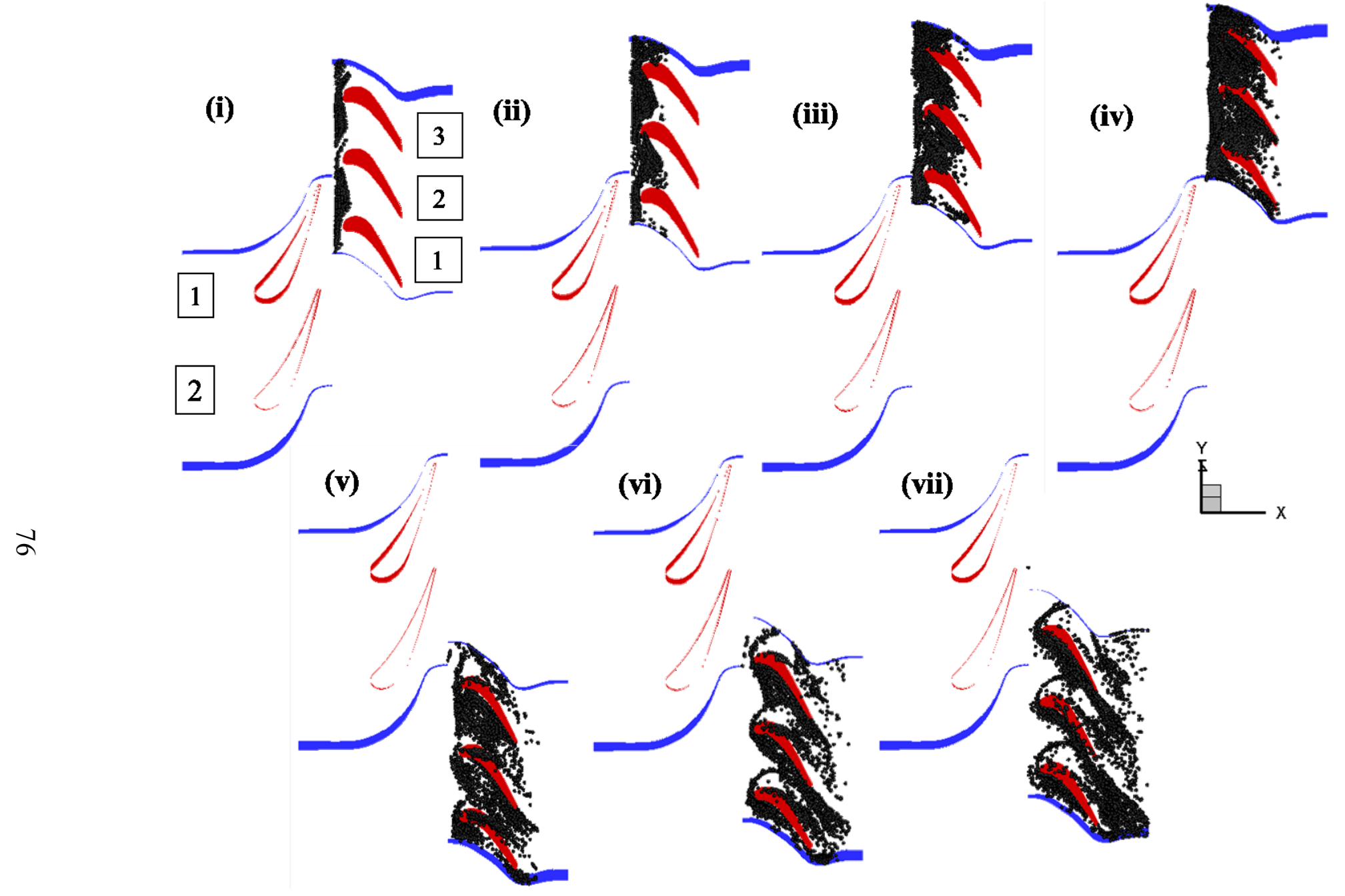

Figure 3.21: Tip gap (97-100\% span), unsteady $10 \mu \mathrm{m}$ particle locations at (i) $t=\Delta t+t_{0}$, (ii) $t=6 \Delta t+t_{0}$, (iii) $t=11 \Delta t+t_{0}$, (iv) $t=16 \Delta t+t_{0}$, (v) $t=21 \Delta t+t_{0}$, (vi) $t=26 \Delta t+t_{0}$, (vii) $t=31 \Delta t+t_{0}$ 


\subsubsection{Unsteady Impacts and Deposits, 10 micron}

Figure 3.22 shows impact and deposit contours for the 10 micron unsteady and steady cases. As before the three blade surfaces of the unsteady calculation have been summed in regard to impacts and deposits. As with the 6 micron case, the impact patterns are quite different for the unsteady and steady cases. Generally, the distribution favors the hub side but again we see that the locus of impacts in the unsteady case is still at about $75 \%$ axial chord and on the hub side. This location is very similar to the unsteady 3 and 6 micron cases. Essentially, the unsteady impact loci do not appear to be affected by the Stokes number effects seen in the steady distributions. For the steady cases as Stokes number is increased (see Figure 3.7) the impact loci, which are distributed along the entire chord for the 3 micron case, become much more localized on a small region behind the leading edge near the hub side. This localization does not occur with increasing Stokes number in the unsteady cases. This could be due to the unsteady wake action described in earlier sections, which causes particles to approach the blade pressure surface with a lower incidence angle, causing them to impact at a higher chordwise location.

Figure 3.23 shows impact and deposit contours for the suction surface of the 10 micron unsteady case. There are significant impacts and deposits on the suction surface $(76 \%$ and $0.7 \%$, respectively). Note that all of the steady cases have virtually no suction side impacts or deposits. The small regions circled in purple are most likely regions of particles trapped in the boundary layer, repeatedly impacting the wall in a nonphysical 
way. The impact and capture efficiencies were adjusted for those locations by excluding any cells that had a number of impacts above the maximum value on the rest of the blade. This threshold was set at 125 impacts per cell.

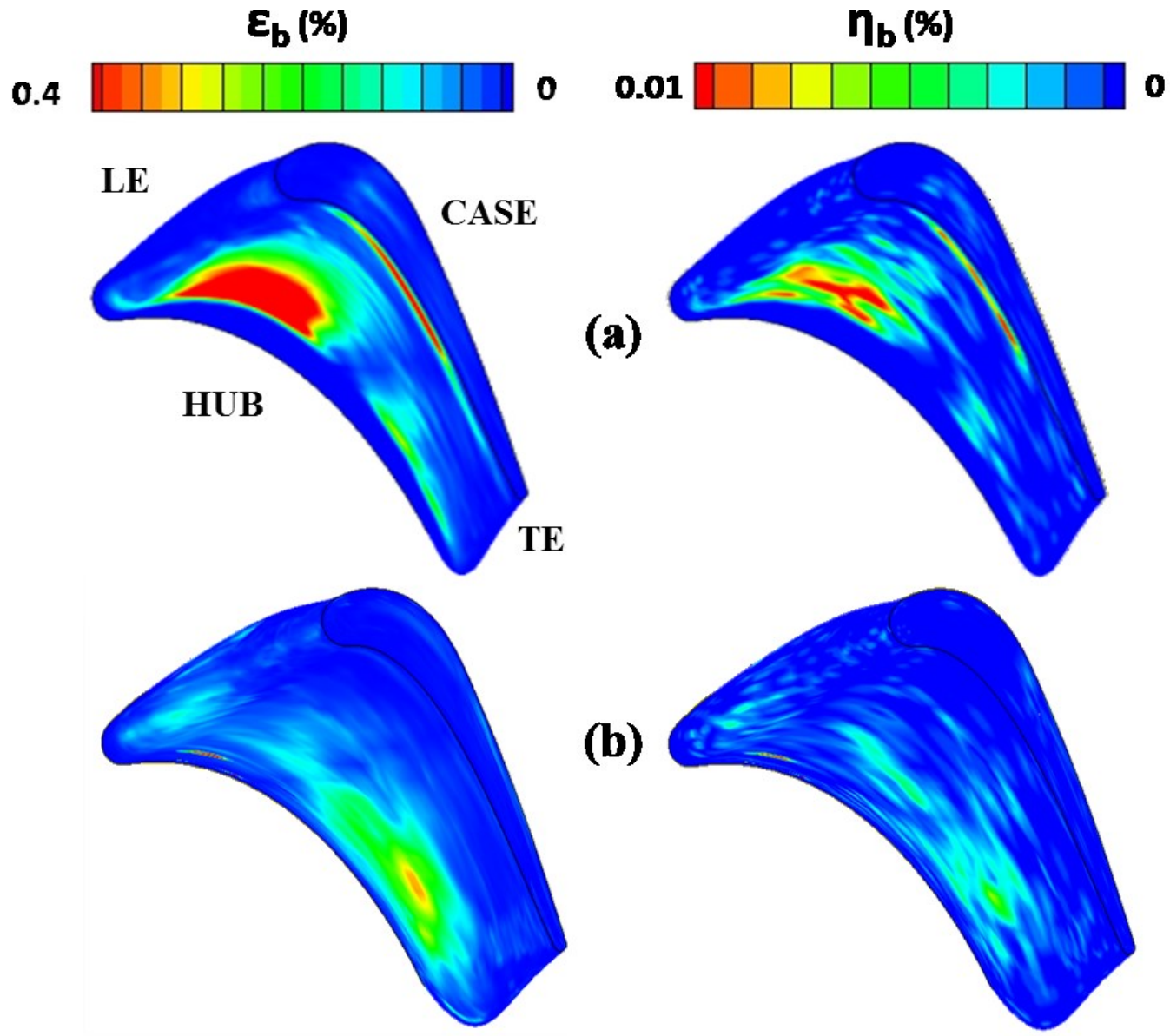

Figure 3.22: Aggregate PS impacts and deposit contours for $10 \mu \mathrm{m}$ particles (a) steady, (b) unsteady 


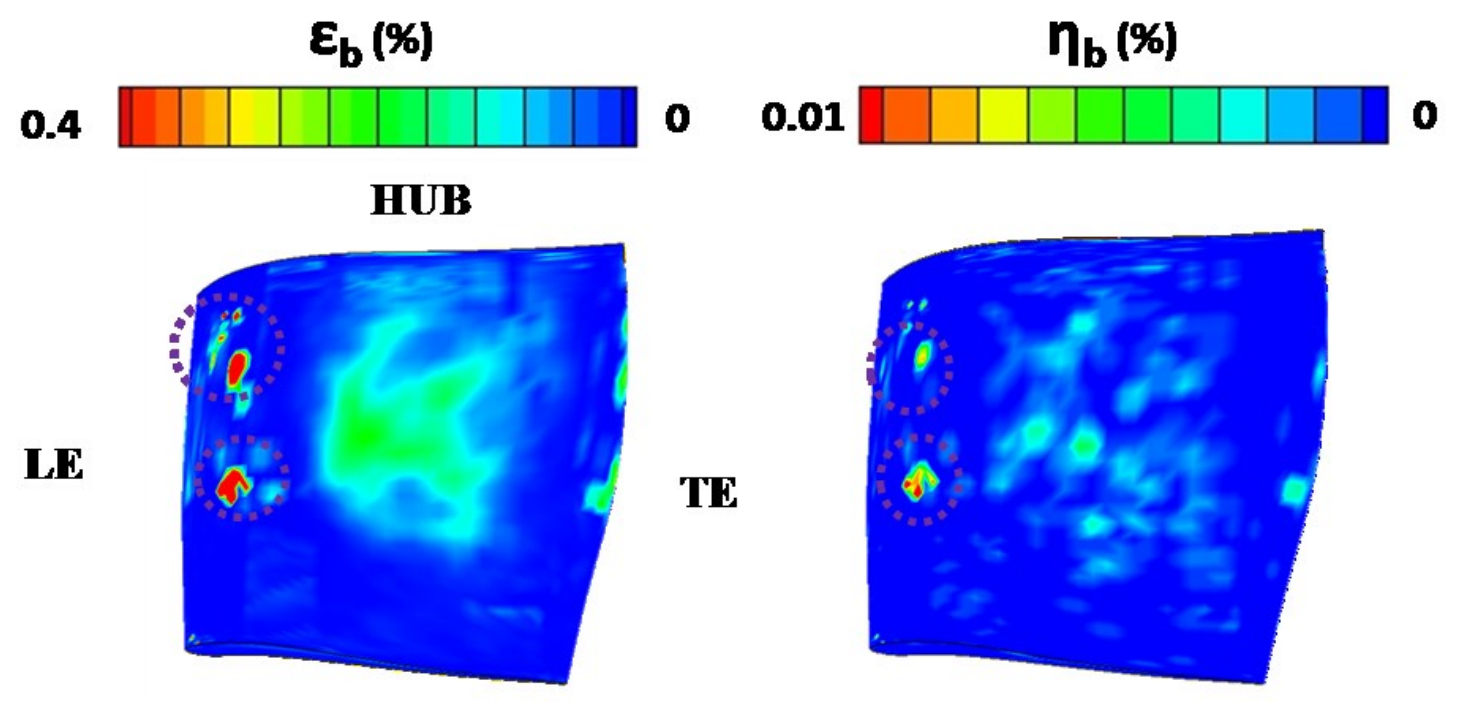

CASE

Figure 3.23: Aggregate SS impact and capture efficiency contours, $10 \mu \mathrm{m}$ unsteady

\subsubsection{Low Rotational Speed, Steady Case}

In order to add some further evidence to the explanation of unsteady particle motion in sections 3.2.1 and 3.2.2, a steady mixing plane solution was calculated using a lower rotational speed than the other cases $(1000 \mathrm{rad} / \mathrm{s}$ instead of $1274 \mathrm{rad} / \mathrm{s})$. This was done to demonstrate the effect of the relative frame transformation on particle motion.

Specifically, the lower rotational speed will cause the relative velocity vector, when transformed by subtracting the rotational velocity, to have a higher incidence angle on the blade surface. 
Figure 3.24 below demonstrates an example of the velocity triangles for two different rotational speeds. Note that particles with a higher incidence angle, in the low rotational speed case, should be more likely to impact the blade pressure surface due to the need for a larger trajectory correction in order to avoid contact.

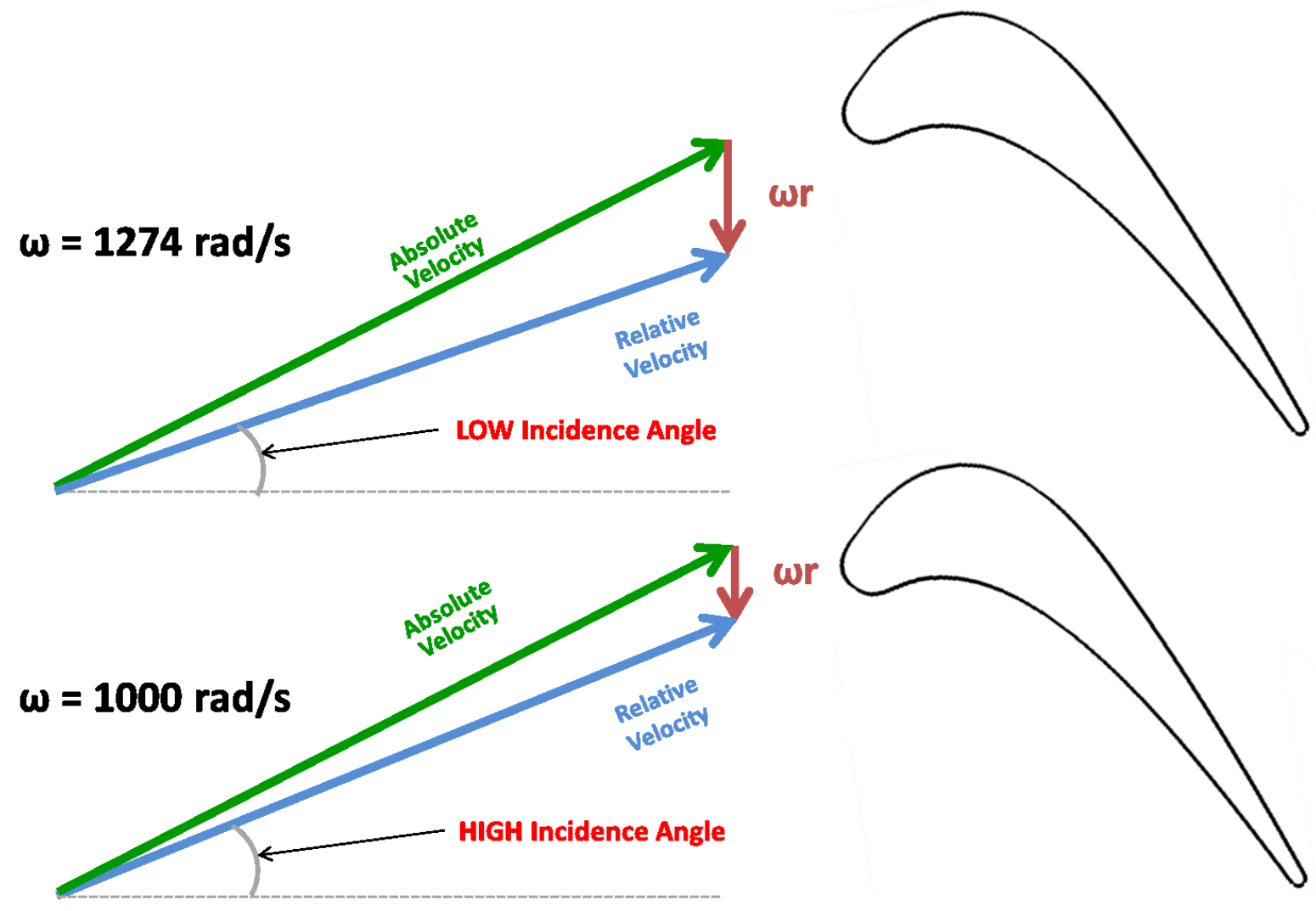

Figure 3.24: Example velocity triangles for two different blade rotational speeds 
Figure 3.25 shows particle pathlines for the low and baseline rotational speed cases. It can be seen that the velocity triangle argument above holds true as more particles enter the blade domain at a higher incidence angle to the blade. The area circled in red shows particles deviating from streamlines and passing through the periodic boundary before entering the blade domain at a very steep angle.

(a)

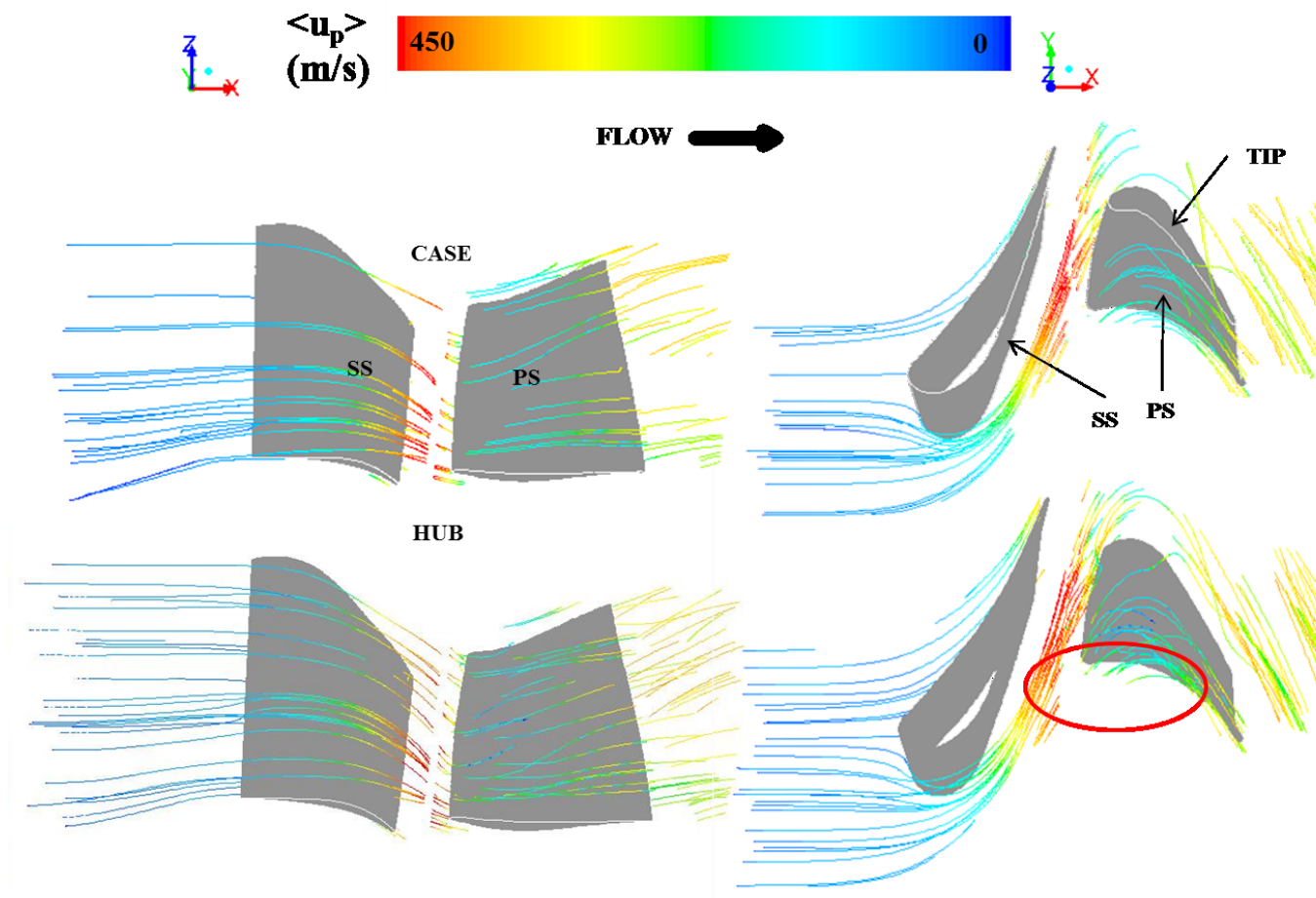

Figure 3.25: Particle tracks for (a) $1274 \mathrm{rad} / \mathrm{s}$ and (b) $1000 \mathrm{rad} / \mathrm{s}, 3 \mathrm{micron}$ particles

Figure 3.26 shows impact and deposit contours for the low and baseline rotational speed cases. As expected, it can be observed that the low rotational speed case does indeed experience a larger number of impacts on the pressure surface of the blade and subsequently more deposits in that location as well. 


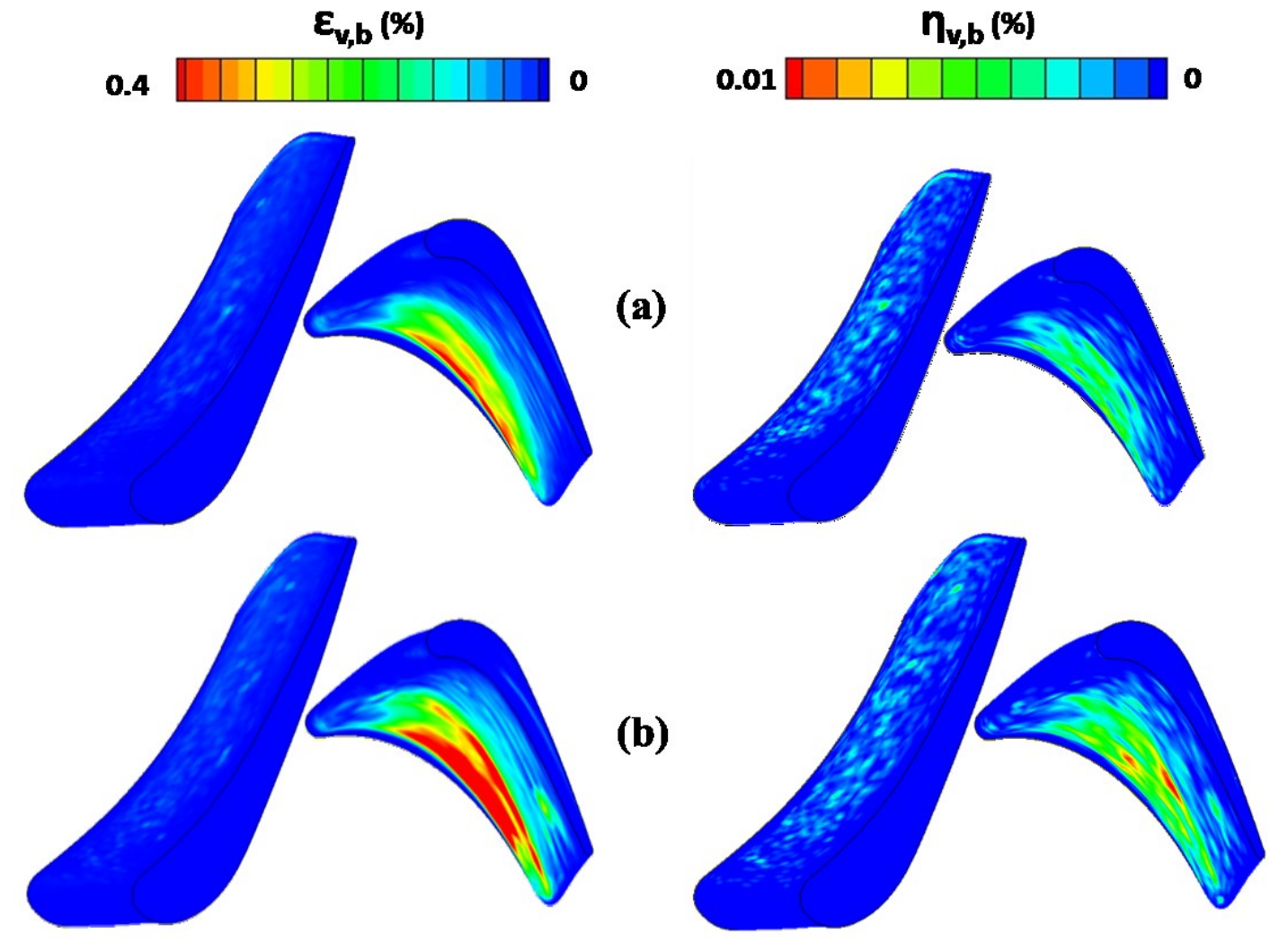

Figure 3.26: Impact and capture efficiency contours for (a) baseline rotational speed and (b) low rotational speed, $3 \mu \mathrm{m}$

The evidence of this low speed case gives some support to the unsteady wake argument made in section 3.2.1 and 3.2.2. One can see that the transformation to the relative frame does indeed have an effect on particle motion. Based on the fact that increasing the rotational speed, shown in (a) of Figure 3.26, caused lower particle incidence angles and less pressure surface impacts in this steady case, it is reasonable that the lower velocity of the vane wake (which contains the majority of the particles) would also cause a lower incidence angle and a migration away from the blade pressure surface in the unsteady case. 


\subsection{Impact and Deposit Metrics}

In order to quantify the behavior of entrained particles in the flow through the turbine stage two non-dimensional parameters are defined: impact efficiency and capture efficiency.

$$
\begin{gathered}
\varepsilon_{v}=\frac{N_{i, v}}{N_{i n j, v}}, \varepsilon_{b}=\frac{N_{i . b}}{N_{i n j, b}} \\
\eta_{v}=\frac{N_{d, v}}{N_{i n j, v}}, \eta_{b}=\frac{N_{d . b}}{N_{i n j, b}}
\end{gathered}
$$

These quantities represent the percentage of particles that enter the domain that eventually impact or deposit on a given surface, respectively. For example a capture efficiency of $100 \%$ on the vane signifies that all of the particles that entered the vane domain were deposited on the vane surface. Note that $N_{i n j, v}$ is the total number of particles injected at the vane inlet plane in the calculation of $\eta_{v}$, while $N_{i n j, b}$ is the number of particles that enter the blade inlet in the calculation of $\eta_{b}$. Figure 3.27 summarizes impact and capture efficiencies for the vane and blade using both mixing plane particle tracking methods (averaged and preserved) for all particle diameters. 


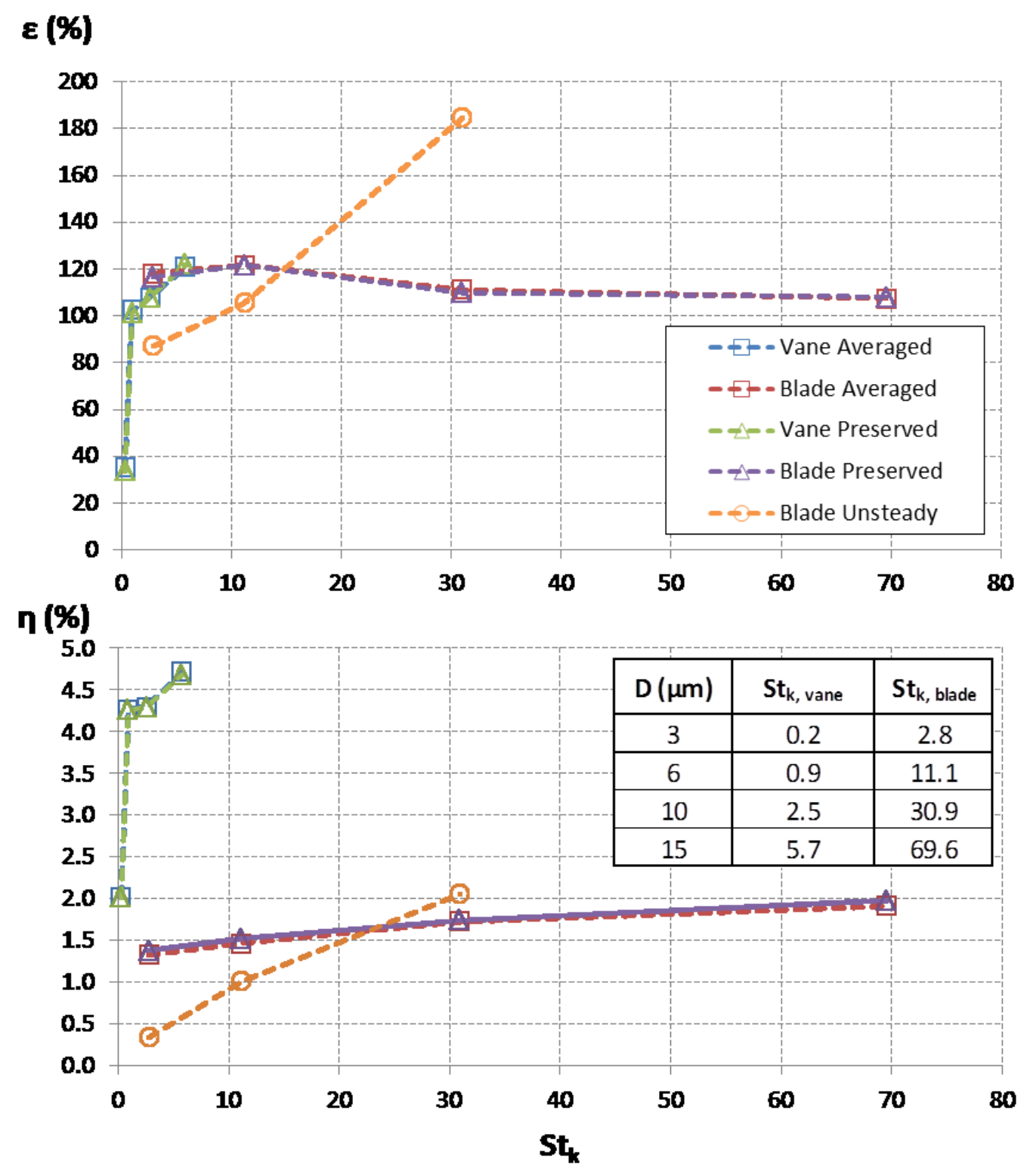

Figure 3.27: Impact and capture efficiencies vs. Stokes number

Increasing particle size has a larger effect on the vane capture efficiency due to the higher range of Stokes number ranges in the blade (2.8-70) compared to the vane (0.2-5.7). 


$$
S t_{k}=\frac{\rho_{p} d_{p}^{2} V_{i}}{18 \mu_{T_{p}} l_{c}}
$$

Stokes number is defined as the ratio of the characteristic particle time to a characteristic time of the flow or obstacle. Larger particles require more time to react to changes in the flow and thus have a higher Stokes number. The leading edge diameters of the vane and blade were used as the characteristic length scales for each domain. The average inlet velocity for each domain was used as the characteristic velocities. It should be noted that for the steady calculations the blade impact efficiencies and capture efficiencies are not very sensitive to particle size in the range studied, which are very representative of actual ingested particulate. Also, the tendency for the blade to have much lower capture efficiencies despite much larger impact efficiencies at all sizes is due to the falling temperature of the expanding flow as it progresses through the stage.

Due to time constraints, only three of four particle sizes were investigated with the fully unsteady solution. Results for this case are also shown in Figure 3.27. Recall that the results are not presented for the unsteady vane case since these particle trajectories were not computed in this flow — they should be very similar to the steady values. The steady calculations are shown to over-predict impacts, with respect to the unsteady results, at particle sizes of 3 and 6 micron by $30 \%$ and $15 \%$, respectively. This finding agrees with the results of [11] where the steady mixing plane was found to over predict erosion in a full-stage draft fan. These results show that the unsteady calculations' inclusion of the 
discrete vane wakes and their relative motion with the blades is crucial in accurately predicting particle trajectories, impacts, and ultimately deposits in the rotor.

However, it is very interesting to note that at the larger Stokes number (10 micron diameter) the unsteady solution predicts $75 \%$ more impacts than the steady case. As was shown in Section 3.2, this can be attributed to impacts on the pressure surface that in turn rebound on the suction surface of the neighboring blades, greatly elevating the impact efficiency. These rebounds on the suction surface did not occur at all in the steady case and it has been postulated that they can be attributed to the timing of impacts with passage Mach number unsteadiness as shown in Figure 3.19, as well as a shifted impact locus closer to the blade leading edge. The timing of impacts with passage unsteadiness could cause particles to experience a lower amount of drag after impacting and to not be re-entrained readily as is seen in the steady case (Figure 3.20), allowing them to traverse the passage and strike the suction surface. The shifting of the impact locus closer to the blade leading edge in the steady cases gives particles a longer, more steeply angled trajectory to follow in order to the reach the suction surface - the location of the unsteady impact locus is near the trailing edge where the distance and angle needed to contact the suction surface are smaller. More investigation is needed to determine if these hypotheses are the cause of the suction side impacts.

Because the differences in the 6 and 10 micron impact and capture efficiencies are due to the suction side impacts it's useful to parse the impacts and deposits with and without the suction side impacts. Figure 3.28 shows the addition of unsteady data with only PS and 
tip impacts and deposits accounted for-SS values are removed. More investigation of the rebound phenomena is needed but it is interesting to note that the removal of the suction side impacts causes the 10 micron impact efficiency to be almost identical to that of the steady case and makes the 3 and 6 micron cases very similar.

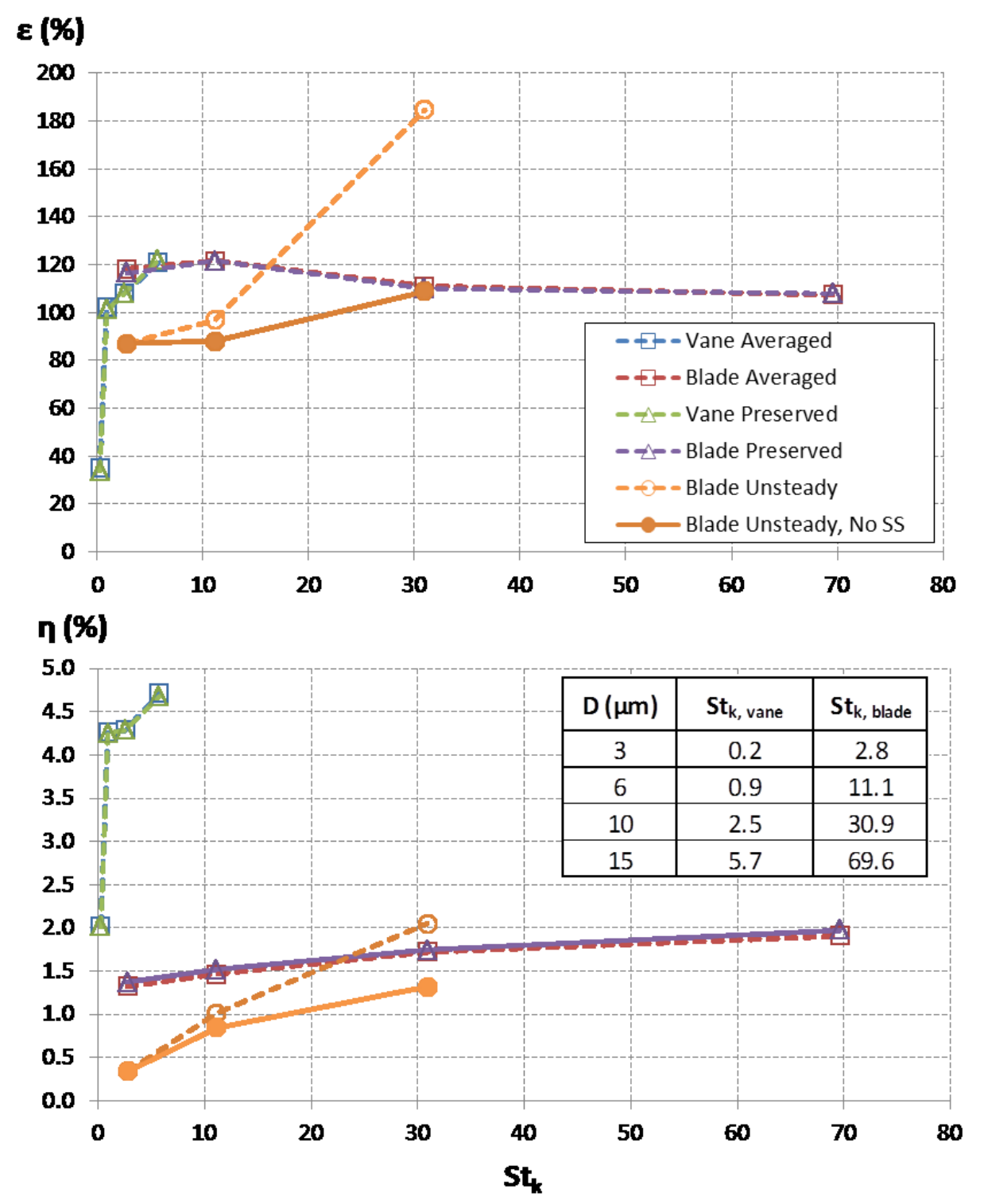

Figure 3.28: Impact and capture efficiencies vs. Stokes number, SS values removed 


\section{Chapter 4: Conclusions}

Deposits and impacts are analyzed for a turbine stage using a steady, mixing plane calculation with two different particle averaging techniques: averaged and preserved. The two methods yielded almost identical impact characteristics, deposit characteristics, and particle tracks due to low amounts of circumferential variation in temperature and velocity. Increasing particle size increased capture efficiencies for both vane and blade surfaces and caused a localization of deposits on the trailing edge of the vane pressure surface. Similar effects were seen on the blade as increased particle size caused a localization of deposits on the hub side, pressure surface behind the leading edge and also at the very tip of the pressure surface. This is due to the more ballistic nature of large particles and their tendency to enter the blade domain at a higher incidence angle towards the hub side and be centrifuged radially towards the tip. The tip deposits are of serious concern as they would likely cause reductions in cooling effectiveness in an area that is difficult to cool and may add to tip losses. Deposits at the vane trailing edge, which is near the throat of the passage, could reduce the flow through area of the throat causing a reduction in choked mass flow. This in turn would reduce the overall mass flow through the engine causing a reduction in max power.

The steady calculations were found to produce impact and deposition results that deviate from the unsteady calculations: 3 and 6 micron particle steady calculations over 
predicted impact efficiencies by $30 \%$ and $15 \%$, respectively. These results are evidence that including the discrete unsteady vane wake movement when considering particle trajectories is critical. At the 10 micron particle diameter the steady solution underpredicted the impact efficiency by $75 \%$. The steady 10 micron's underprediction is attributed to rebounds from the pressure surface that strike the suction surface of the neighboring blade - these do not occur in the steady solution due to their tendency to impact the pressure surface close to the leading edge where they have a less steep rebound angle and a longer distance to travel to the adjacent suction surface. The steady solution also suffers from averaging of the vane wake which eliminates any velocity deficits that would influence particles away from the blade pressure surface. The distribution and location of impacts was found to be quite different for steady and unsteady cases: steady cases showed localization of impact locations near the leading edge hub side with increasing Stokes number while unsteady cases showed a locus that only varied in magnitude but remained near the trailing edge hub side pressure surface. This is also attributed to the deficit of vane wake velocity which causes particles to approach the pressure surface at shallower incidence angles. All of these results were performed at near typical particulate concentration for flight conditions and are useful in guiding designers or researchers of turbomachinery in best practices for analyzing turbine designs. In general, the study suggests that despite the large increase in computational cost, the unsteady results differ from steady approximations in impact and capture efficiencies and in deposit distributions and are necessary to attain the most accurate prediction of deposition phenomenon in a high pressure turbine. 
Future work on this topic should include the largest particle size for the timeintensive unsteady calculations to further map out the effect of Stokes number on unsteady particle tracking. A study of different rotor speeds would also be enlightening given that any wake motion due to the relative transformation of absolute vectors will be enhanced or diminished at different rotational speeds. Also, because the deviation from unsteady and steady was found to be greatly dependent on particle rebounds, a different deposition model could be used that may trap more particles before rebounding. This may bring steady and unsteady methods closer together in their impact and capture efficiencies at higher Stokes numbers. Another relevant addition would be including non-uniform temperature inlet boundary conditions like hot streaks experienced by real turbine hardware from discrete combustor fuel injectors. Because deposition is known to be heavily dependent on particle temperature, these flow temperature non-uniformities would have a first order effect on the locations of deposits. Also, temperature nonuniformities cause velocity differences in the flow, similar to those found in the wake fluid of the vane. These velocity differences have been shown to effect wake motion through the blade passage and would be exacerbated by temperature non-uniformities. The addition of film cooling to vanes and/or blades would make these calculations more realistic and would have an effect on particle motion and temperatures and ultimately deposition results. 


\section{References}

[1] Van Donkelaar, A. (2010). Global satellite-derived map of $\mathrm{PM}_{2.5}$ averaged over 2001-2006 [Online image]. Retrieved January 1, 2015 from http://www.nasa.gov/topics/earth/features/health-sapping.html.

[2] Bons, J., 2010. “A Review of Surface Roughness Effects in Gas Turbines”. ASME J. Eng. Gas Turb. Power, 132.

[3] Dunn, M.G., "Operation of Gas Turbine Engines in an Environment Contaminated with Volcanic Ash," J. Turbomachinery, Vol. 134, 051001 Sept. 2012.

[4] Dunn, M., Baran, A., and Miatech, J., 1996. "Operation of Gas Turbine Engines in Volcanic Ash Clouds". ASME J. Eng. Gas Turb. Power, 118.

[5] Tabakoff, W., Hamed, A., and Metwally, M., 1991. "Effect of Particle Size Distribution on Particle Dynamics and Blade Erosion in Axial Flow Turbines". ASME J. Eng. Gas Turb. Power, 113.

[6] Hamed, A. A., Tabakoff, W., Kaushik, D., Rivir, R., and Puneet, A., 2004. "Turbine Blade Surface Deterioration by Erosion". Presented at the 2004 ASME Turbo Expo in Vienna, Austria. Paper Number: GT2004-54328.

[7] Bidwell, C., 2012. "Ice Particle Transport Analysis with Phase Change for the E3 Turbofan Engine Using LEWICE3D Version 3.2". Presented at the 2012 AIAA Atmospheric Space Environments Conference in New Orleans, Louisiana. Paper Number: 2012-217700.

[8] Suzuki, M., Kazuaki, I., and Makoto, Y., 2008. "Numerical Simulation of Sand Erosion Phenomena in Rotor/Stator Interaction of Compressor". Journal of Thermal Science, 17, pp. 125-133. 
[9] Ghenaiet, A., 2014. "Study of Particle Ingestion Through Two-Stage Gas Turbine”. Presented at the 2014 ASME Turbo Expo in Dusseldorf, Germany. Paper Number: GT2014-25759.

[10] Corsini, A., Rispoli, F., Sheard, A., and Venturini, P., 2013."Numerical Simulation of Coal Fly-Ash Erosion in an Induced Draft Fan". Journal of Fluid Engineering, 135.

[11] Yang, H., and Boulanger, J., 2011. "The Whole Annulus Computations of Particulate Flow and Erosion in an Axial Fan". Presented at the 2011 ASME Turbo Expo in Vancouver, Canada. Paper Number: GT2011-45992.

[12] Tafti, D., and Sreedharan, S., 2010. "Composition Dependent Model for the Prediction of Syngas Ash Deposition with Application to a Leading Edge Turbine Vane". Presented at the 2010 ASME Turbo Expo in Glasgow, Scotland. Paper Number: GT2010-23655.

[13] Senior, C., and Srinivasachar, S., 1995. "Viscosity of Ash Particles in Combustion Systems for Prediction of Particle Sticking". Energy and Fuels.

[14] Barker, B., Casaday, B., Shankara, P., Ameri, A., and Bons, J., 2013. "Coal Ash Deposition on Nozzle Guide Vanes: Part II Computational Modeling”. J. Turbomachinery, 135.

[15] Webb, J., Barker, B., Bons, J., Gledhill, A., and Padture, N., 2011. "Coal Ash Deposition on Nozzle Guide Vanes: Part I Experimental Characteristics of Four Coal Ash Types". Presented at the 2011 ASME Turbo Expo in Vienna, Austria. Paper Number: GT2011-45894.

[16] Casaday, B., Prenter, R., Bonilla, C., Lawrence, M., Clum, C., Ameri, A., and Bons, J., 2013. "Deposition with Hot Streaks in an Uncooled Turbine Vane Passage”. ASME J. Eng. Gas Turb. Power, 135. 
[17] Suman, A., Rainer, K., Nicola, A., Mirko, M., Brun, K., Pinelli, M., and Pier Ruggero, S., 2014. "Quantitative CFD Analyses of Particle Deposition on a Transonic Axial Compressor Blade, Part I: Particle Zones Impact". Presented at the 2014 ASME Turbo Expo in Dusseldorf, Germany. Paper Number: GT201425282.

[18] Haldeman, C., Dunn, M., and Mathison, R., 2010. "Fullycooled Single Stage HP Transonic Turbine: Part I - Influence of Cooling Mass Flow Variations and Inlet Temperature Profiles on Blade Internal and External Aerodynamics". Presented at the 2010 ASME Turbo Expo in Glasgow, Scotland. Paper Number: GT201023446 .

[19] Nickol, J., and Mathison, R., 2014. "Time-resolved Heat Transfer and Surface Pressure Measurements for a Fully- Cooled Transonic Turbine Stage". Presented at the 2014 ASME Turbo Expo in Dusseldorf, Germany. Paper Number: GT2014-26407.

[20] ANSYS, 2009. "Fluent theory guide v 12.0". pp. 433-435.

[21] Woelke, M., "Eddy Viscosity Turbulence Models employed by Computational Fluid Dynamic", Transactions of Institute of Aviation, No. 191.

[22] Ameri, A., Rigby D., 2013. "Effects of Hot Streak and Phantom Cooling on Heat Transfer in a Cooled Turbine Stage Including Particulate Deposition". Workshop Proceedings from University Turbine Systems Research program at the National Energy Technology Laboratory.

[23] Steinthorsson, E., Liou, M.S., and Povinelli, L.A., 1993, "Development of an Explicit Multiblock/Multigrid Flow Solver for Viscous Flows in Complex Geometries," AIAA-93-2380; (NASA TM-106356.)

[24] Morsi, S.A., and A. J. Alexander, "An investigation of particle trajectories in twophase flow systems” J. Fluid Mech., 55(2):193-208, November 1971.

[25] Ranz, W.E., and W. R. Marshall, W.R. Jr., "Evaporation from Drops, Part I". Chem. Eng. Prog., 48(3):141-146, March 1952. 
[26] Dehbi, A., 2006. "Assessment of a New Fluent Model for Particle Dispersion in Turbulent Flows". Workshop Proceedings on Benchmarking of CFD Codes for Application to Nuclear Reactor Safety (CDF4NRS).

[27] Elghobashi, S., 1994. "On Predicting Particle-laden Turbulent Flows". J. Applied Scientific Research, 52, pp. 309-329.

[28] Whitaker, S., Reilly D., Bons, J.P., Crafton, J., “A Survey of Particle Impact Characteristics Using High Speed Particle Shadow Velocimetry" Proceedings from AIAA Fluid Dynamics Conference, San Diego, California, (2013).

[29] N'Dala, I., Cambier, F., Anseau, M., and Urbain, G., 1984. "Viscosity of Liquid Feldspars, Part I: Viscosity Measurements". Br. Ceram. Trans. J., 83, pp. 108112. 


\section{Appendix A - FLUENT User-Defined Function Code for Random Particle Injection}

/*CREATES A RANDOMLY DISPERSED INJECTION FILE FOR VANE INLET TO AVOID END WALL BIASING OF PARTICLES */

\#include "udf.h"

\#include "math.h"

DEFINE_ON_DEMAND(RandInjection)

\{

FILE $* f p=$ NULL;

Message(“Beginning Random Injection”);

/* Initialization */

/*CHOOSE THESE PARAMETERS*/

int nParticles $=100000 ; / *$ number of particles $* /$

float $\mathrm{T}=300 ; / *$ Particle temperature*/

float $\mathrm{U}=0 ; / *$ Particle temperature*/

float $\mathrm{V}=0 ; / *$ Particle temperature*/ 
float $\mathrm{W}=0 ; / *$ Particle temperature*/

float D $=0.000006 ; / *$ Particle Diameter*/

/*Loop counters*/

int $\mathrm{j}=0$;

int $\mathrm{k}=0$;

int $\mathrm{m}=0$;

real rSq;

/* RANDOM INLET DISTRIBUTION */

/*with initial velocities and temps*/

float newX,newY,newZ,newU,newV,newW,newD,96ewt,r, theta;

$\mathrm{fp}=$ fopen(“RandomInjection.inj”,,a”);

for $(\mathrm{m}=0 ; \mathrm{m}<\mathrm{nParticles} ; \mathrm{m}++)$

\{

$$
\begin{aligned}
& \text { theta }=0.52641923 * \operatorname{rand}() / \operatorname{pow}(2,31)+1.350755687 \\
& \mathrm{r}=0.05899540 * \operatorname{rand}() / \operatorname{pow}(2,31)+0.167389 \\
& \text { new } \mathrm{X}=-0.0381 \\
& \text { new } \mathrm{Y}=\mathrm{r}^{*} \cos (\text { theta })
\end{aligned}
$$




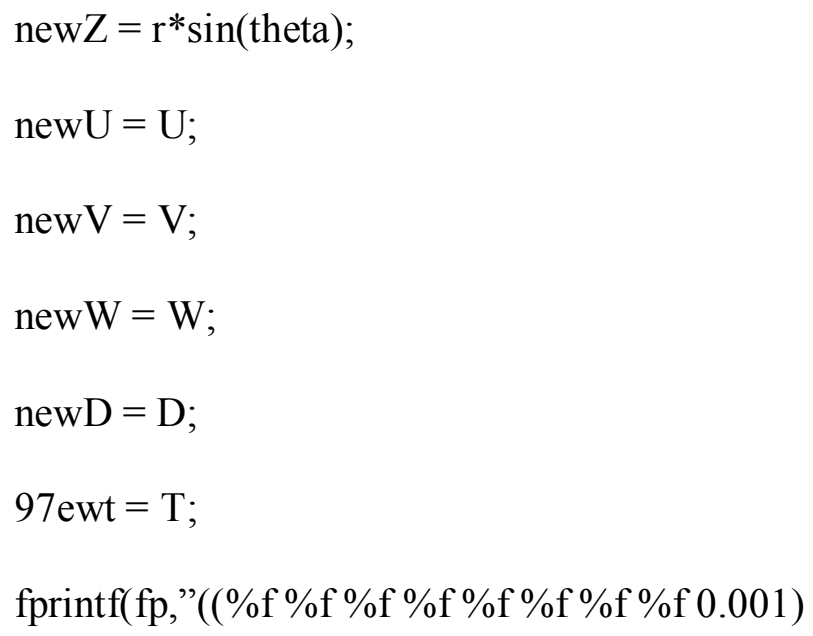

InjectLocations) \n”,newX,newY,newZ,newU,newV,newW,newD,97ewt); \}

fclose(fp);

\} 


\section{Appendix B - FLUENT User-Defined Function Code for Writing Particle Positions at Vane Outlet}

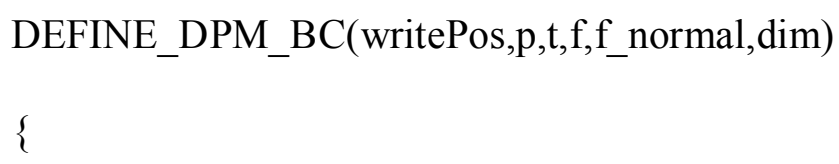




\section{Appendix C - FLUENT User-Defined Function Code for Preserved Mixing Plane Re- Distribution}

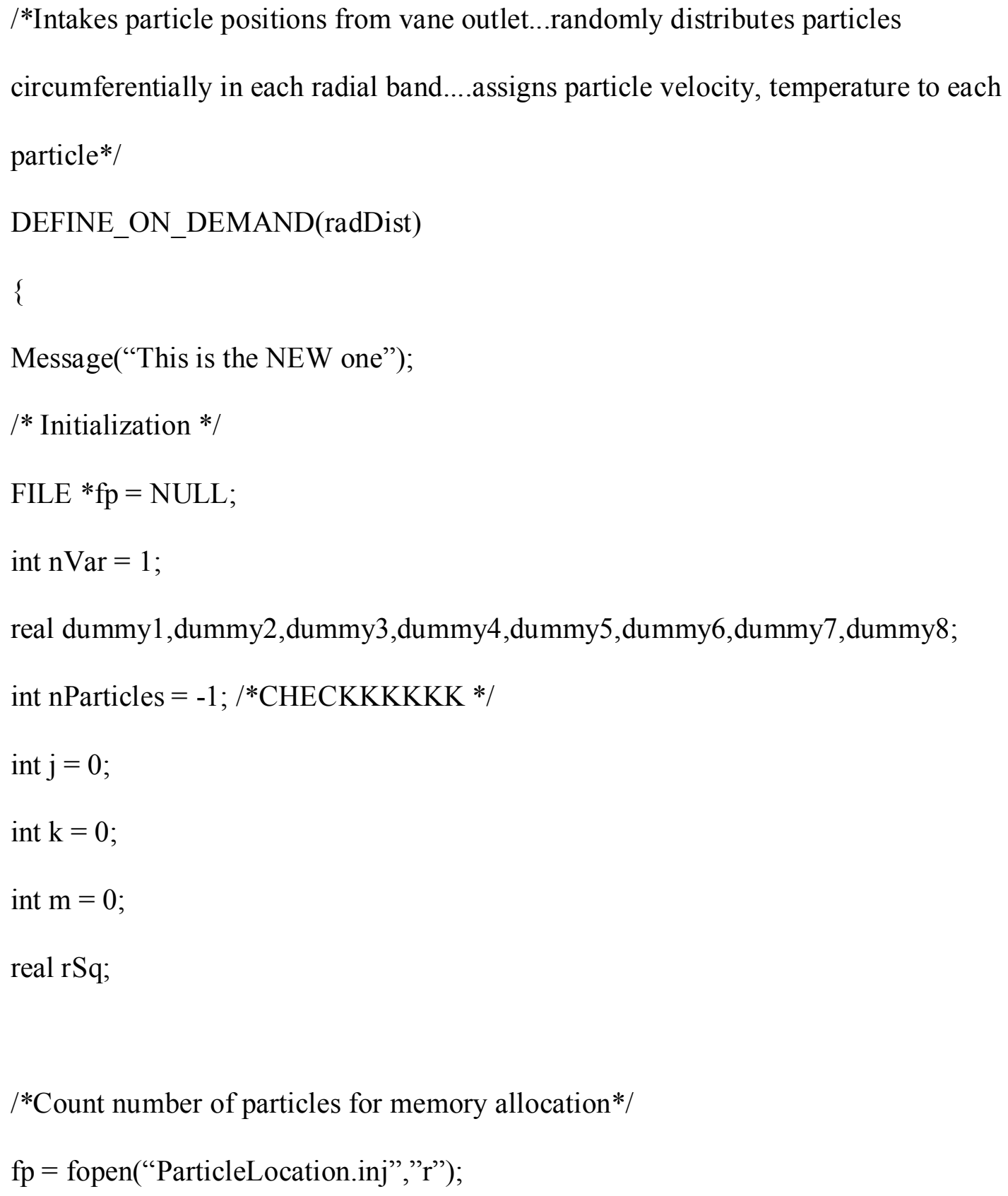




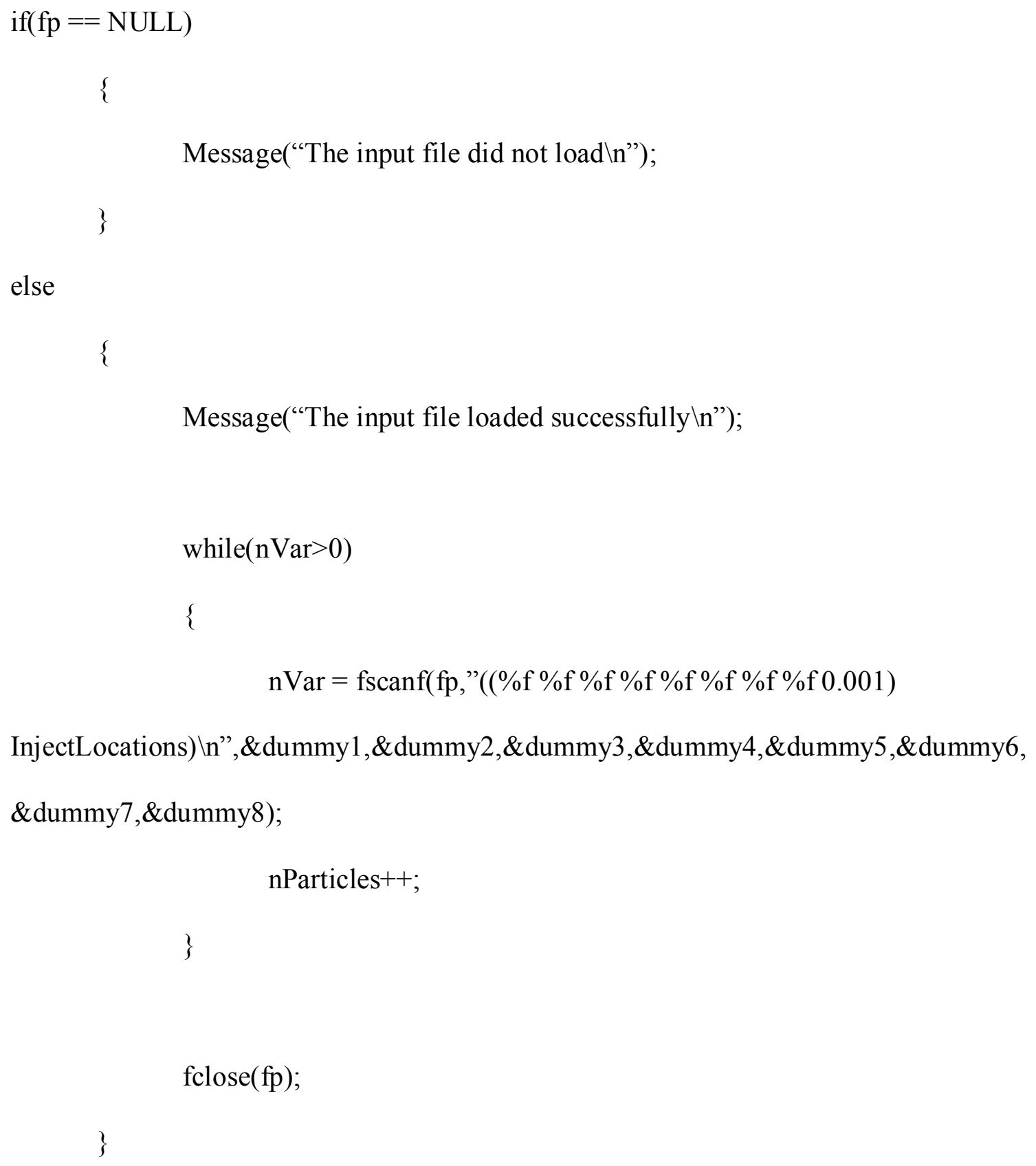


float

$\mathrm{x}[\mathrm{nParticles}], \mathrm{y}[\mathrm{nParticles}], \mathrm{z}[\mathrm{nParticles}], \mathrm{u}[\mathrm{nParticles}], \mathrm{v}[\mathrm{nParticles}], \mathrm{w}[\mathrm{nParticles}], \mathrm{d}[\mathrm{nPartic}$ les],T[nParticles],r[nParticles];

float theta;

$\mathrm{n} \operatorname{Var}=1$;

$\mathrm{fp}=$ fopen("ParticleLocation.inj",,"r");

$\operatorname{if}(\mathrm{fp}==\mathrm{NULL})$

\{

Message(“The input file did not load $\backslash n$ ");

\}

else

Message(“The input file loaded successfully again $\backslash n$ ”);

while $(\mathrm{nVar}>0)$

\{

nVar $=$ fscanf(fp,,$((\%$ f \% \%f \%f \%f \%f \%f \%f 0.001)

InjectLocations) $\backslash n ", \& x[j], \& y[j], \& z[j], \& u[j], \& v[j], \& w[j], \& d[j], \& T[j])$;

$$
\mathrm{j}++ \text {; }
$$

\}

fclose(fp); 


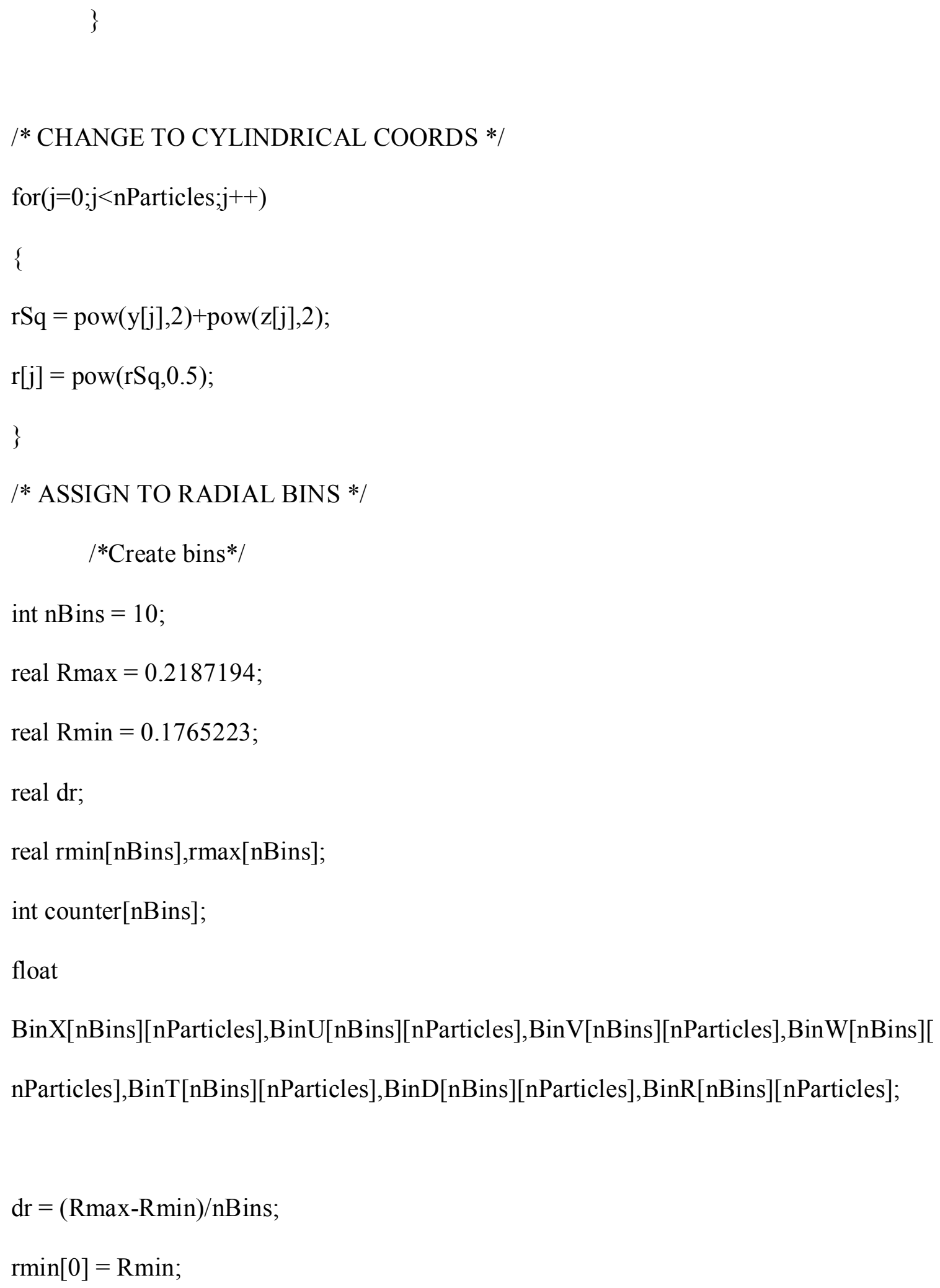




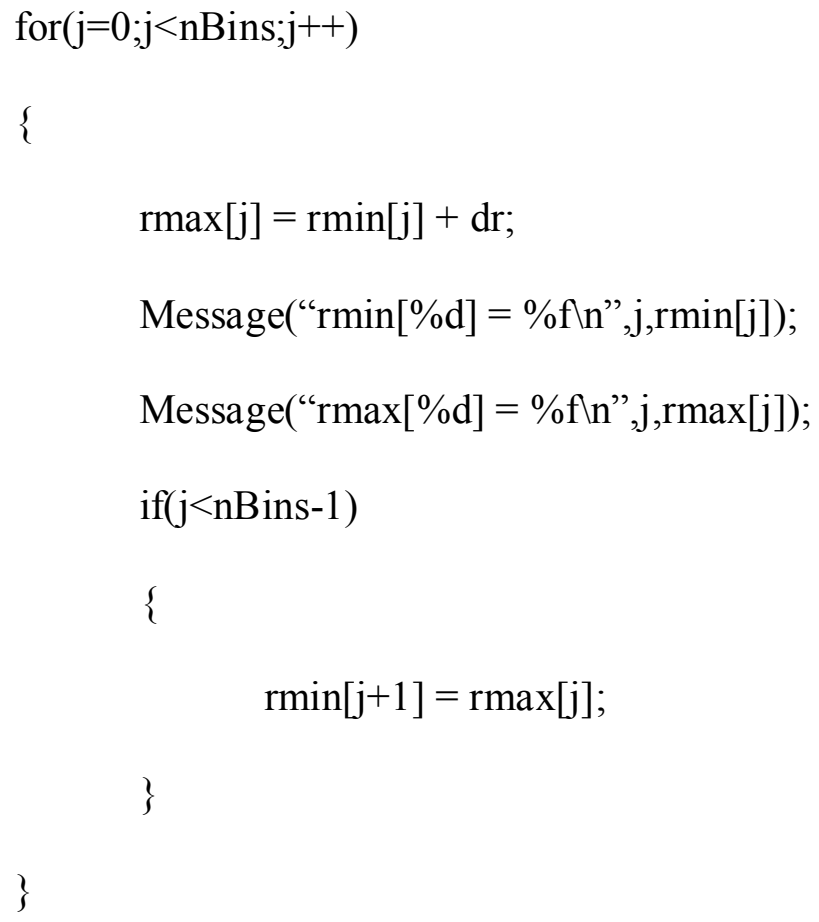




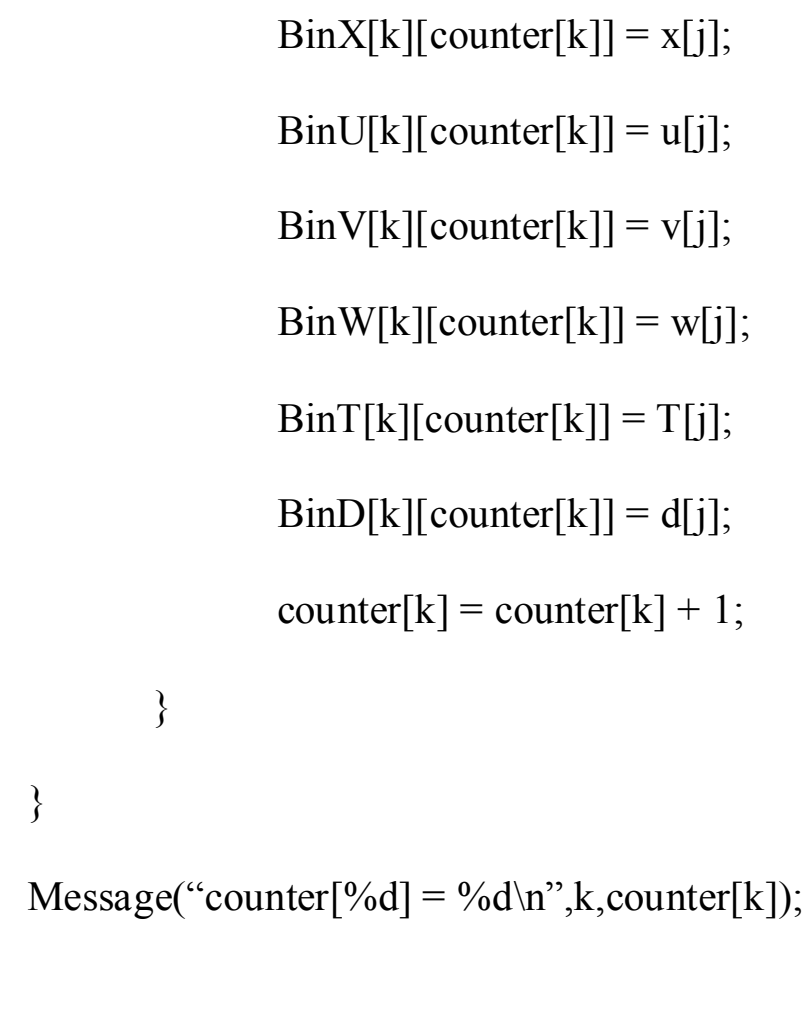




\section{Appendix D - FLUENT User-Defined Function Code for Random Particle Injection and Averaged Mixing Plane Re-Distribution}

/*Intakes particle positions from vane outlet...randomly distributes particles

circumferentially in each radial band....averages particle velocity, temperature in each band and assigns that value to all particles in each band */

\#include "udf.h"

\#include "math.h"

DEFINE_ON_DEMAND(radDistAVG)

\{

Message("This is the NEW one");

/* Initialization */

FILE $* \mathrm{fp}=\mathrm{NULL}$

int $\mathrm{n} \operatorname{Var}=1$;

real dummy1,dummy2,dummy3,dummy4,dummy5,dummy6,dummy7,dummy8; int nParticles $=-1 ; / *$ CHECKKKKKK $* /$

int $\mathrm{j}=0$;

int $\mathrm{k}=0$; 


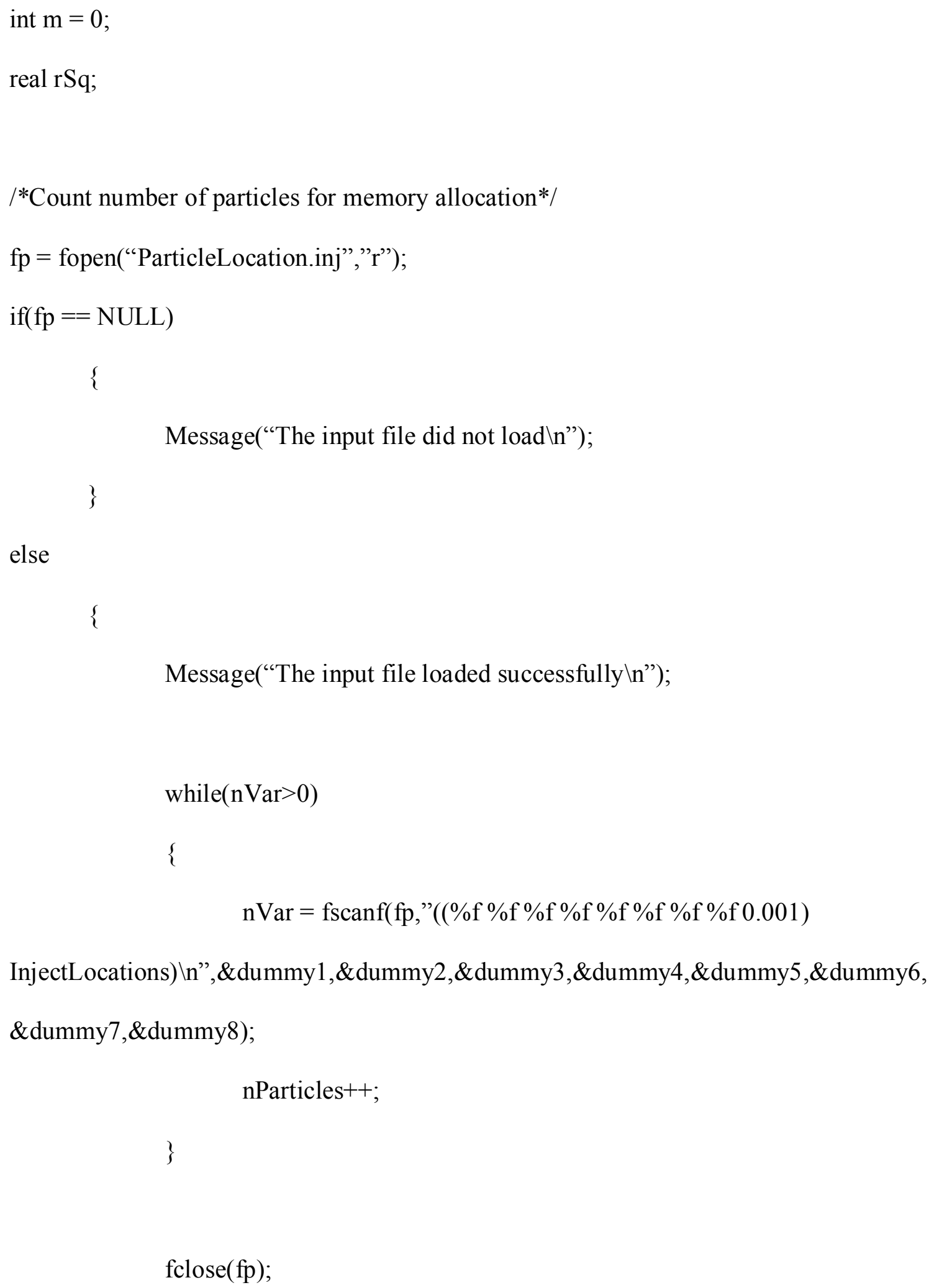


/*Define variables*/

float

$\mathrm{x}[\mathrm{nParticles}], \mathrm{y}[\mathrm{nParticles}], \mathrm{z}[\mathrm{nParticles}], \mathrm{u}[\mathrm{nParticles}], \mathrm{v}[\mathrm{nParticles}], \mathrm{w}[\mathrm{nParticles}], \mathrm{d}[\mathrm{nPartic}$ les],T[nParticles],r[nParticles];

float theta;

$\mathrm{n} \operatorname{Var}=1$;

$\mathrm{fp}=$ fopen("ParticleLocation.inj",,"r");

$\operatorname{if}(\mathrm{fp}==\mathrm{NULL})$

\{

Message(“The input file did not load $\backslash n$ ");

\}

else

Message("The input file loaded successfully again $\backslash n$ ");

while $(n \operatorname{Var}>0)$

\{

$$
\text { nVar }=\text { fscanf(fp, }{ }^{\prime}((\% \text { f \%f \%f \%f \%f \%f \%f \%f 0.001) }
$$

InjectLocations) $\backslash n ", \& x[j], \& y[j], \& z[j], \& u[j], \& v[j], \& w[j], \& d[j], \& T[j])$;

$$
\mathrm{j}++ \text {; }
$$


fclose(fp);

\}

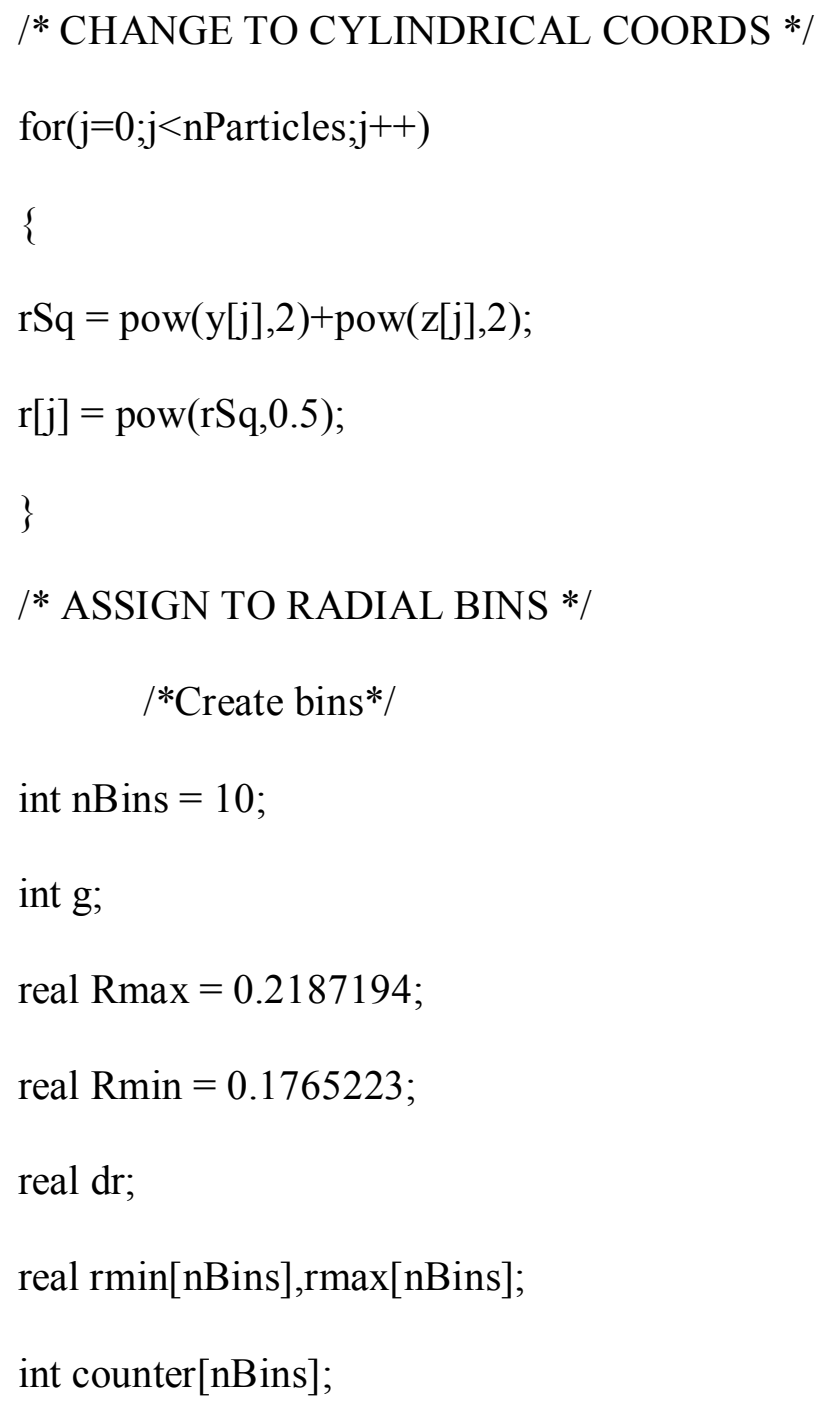




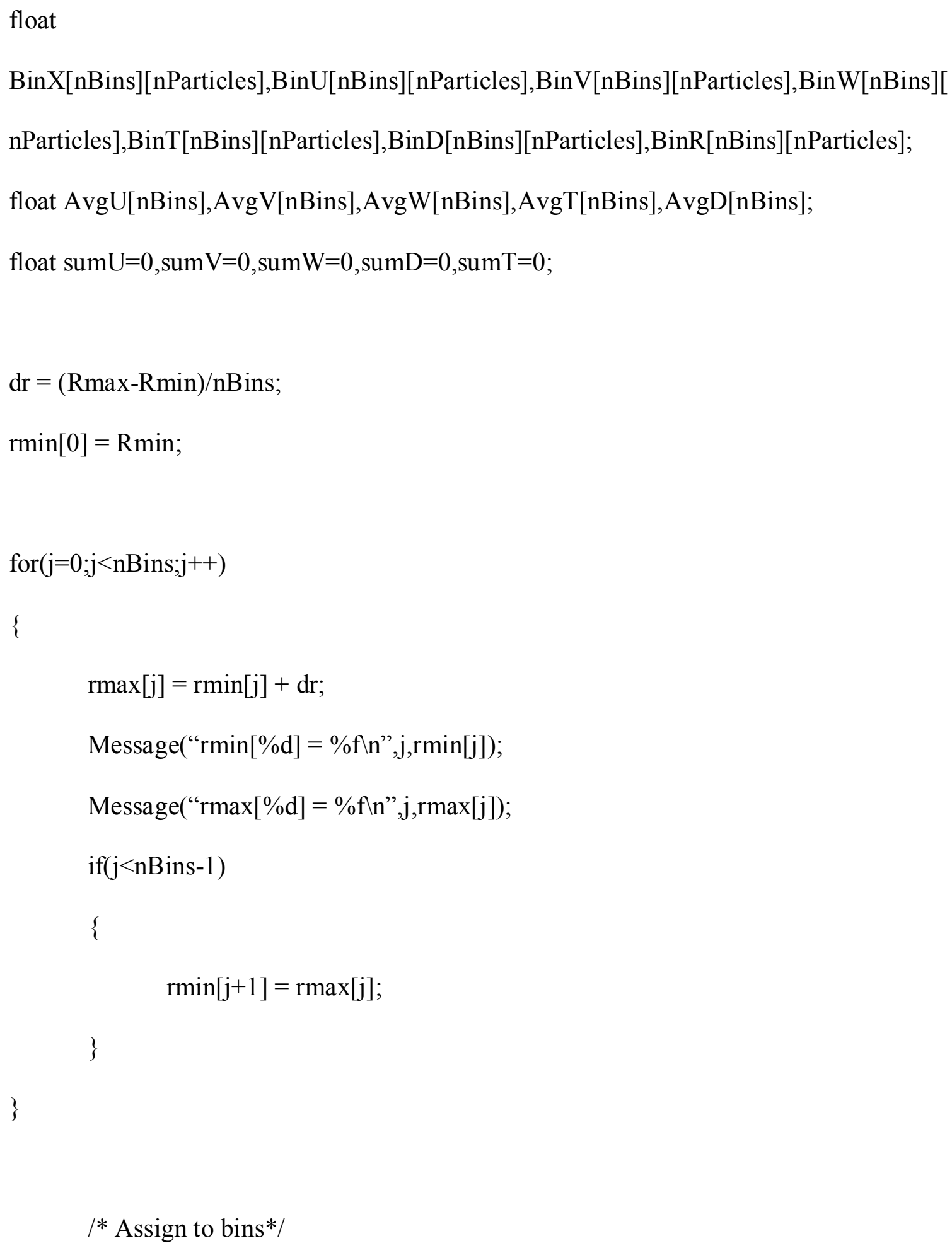




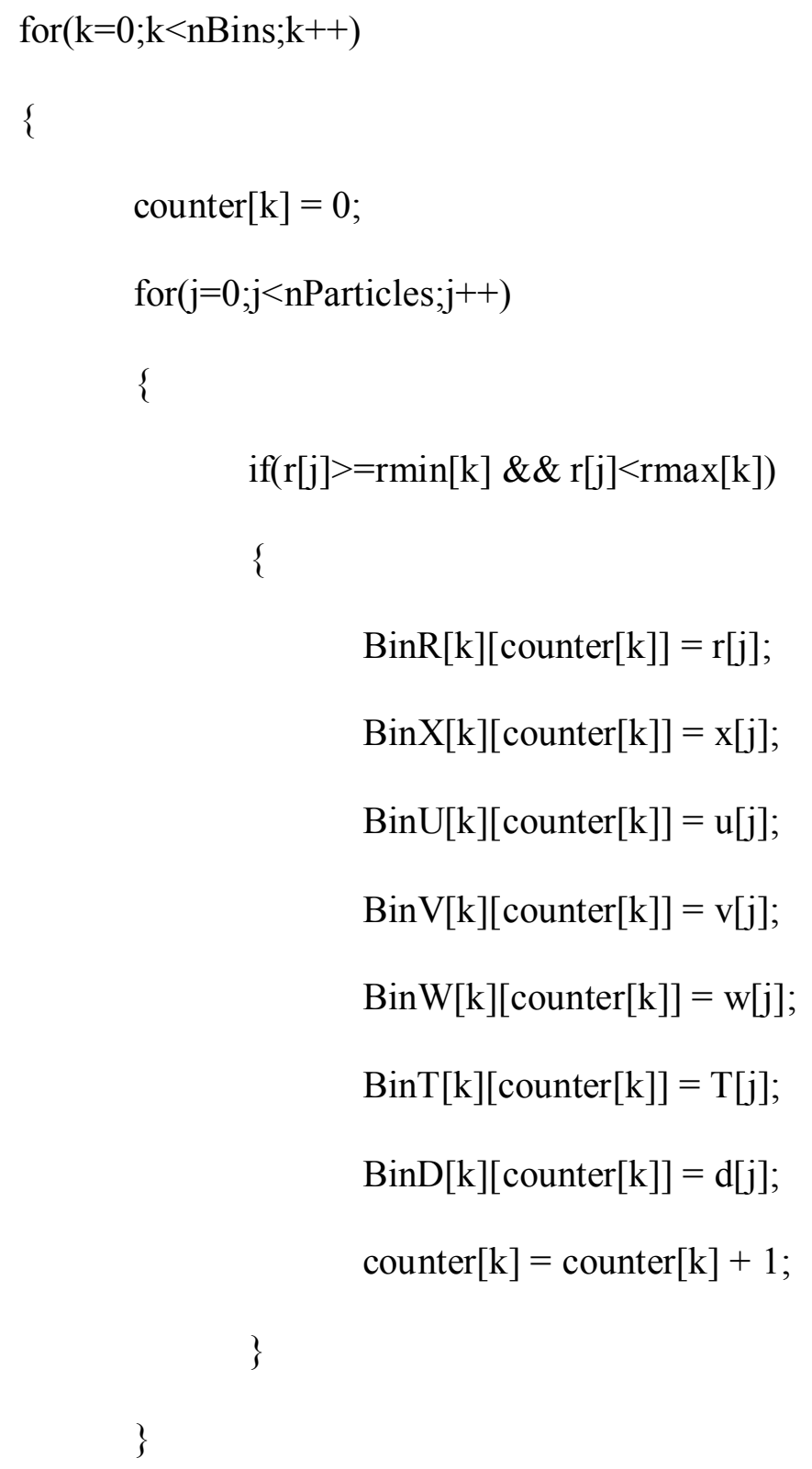




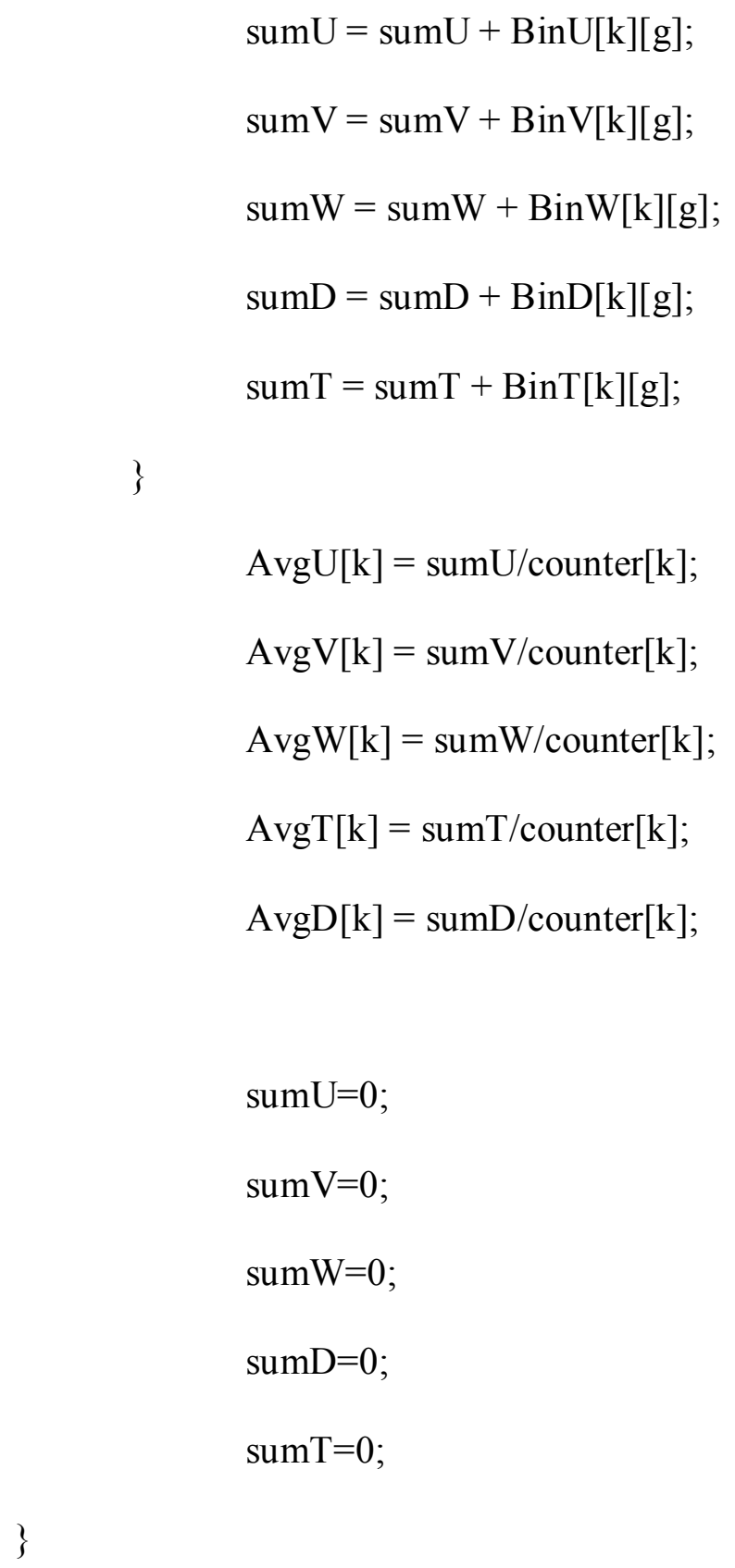


fp = fopen(“DistributedLocationsAVG.inj”,"a");

for $(\mathrm{k}=0 ; \mathrm{k}<\mathrm{nBins} ; \mathrm{k}++)$

\{

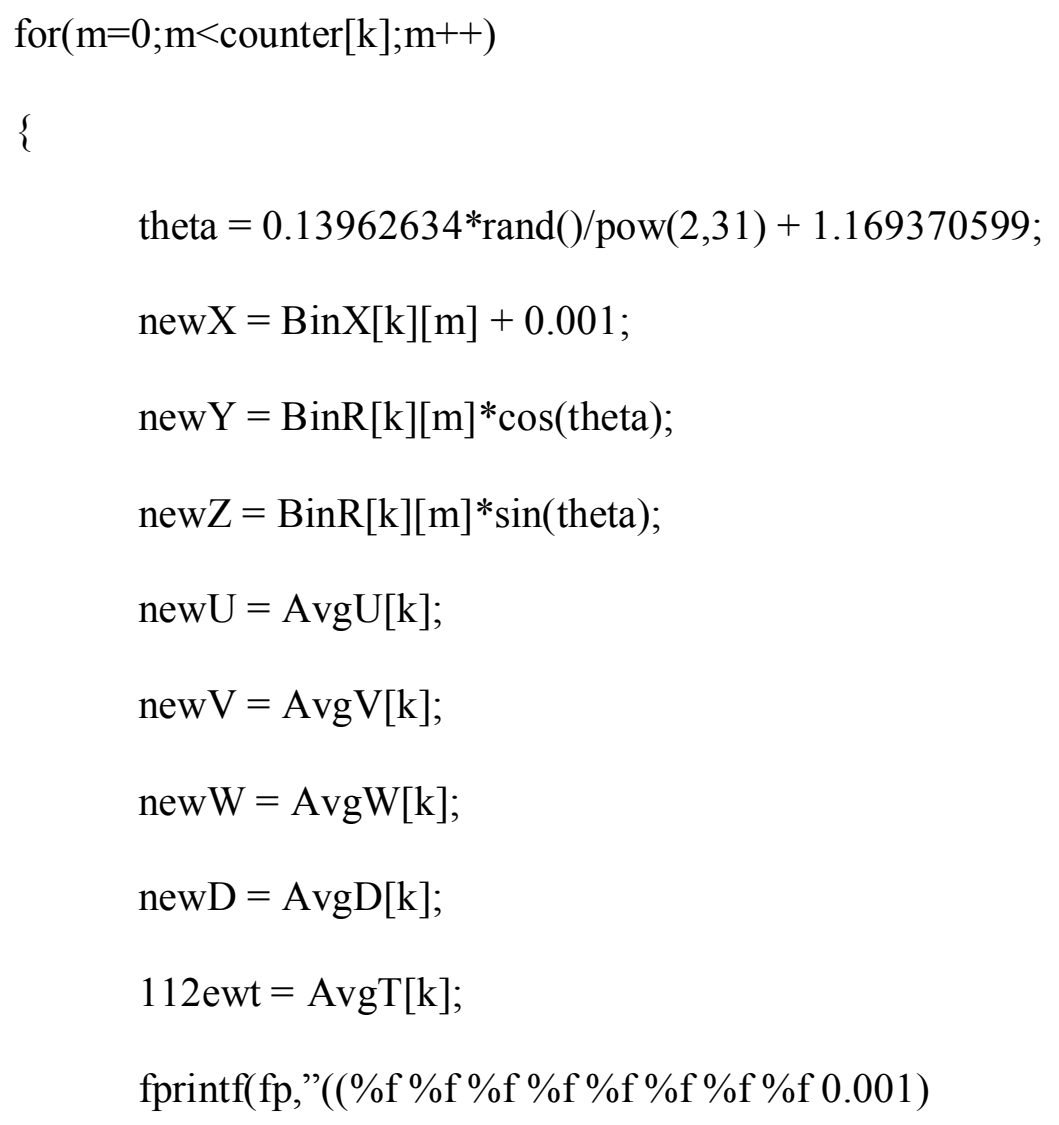




\section{DEFINE_ON_DEMAND(clearUDM) \\ \{}

Thread $*$ t;

Domain *d;

cell_t c;

face_t $\mathrm{f}$;

Message(“New \n");

$\mathrm{d}=$ Get_Domain(1);

Message("Resetting UDMs \n");

thread_loop_c(t,d)

\{

begin_c_loop(c,t)

\{

C_UDMI $(\mathrm{c}, \mathrm{t}, 0)=0.0$;

\}

end_c_loop $(\mathrm{c}, \mathrm{t})$

\}

\} 
Appendix E: Averaged Mixing Plane Particle Redistribution Results

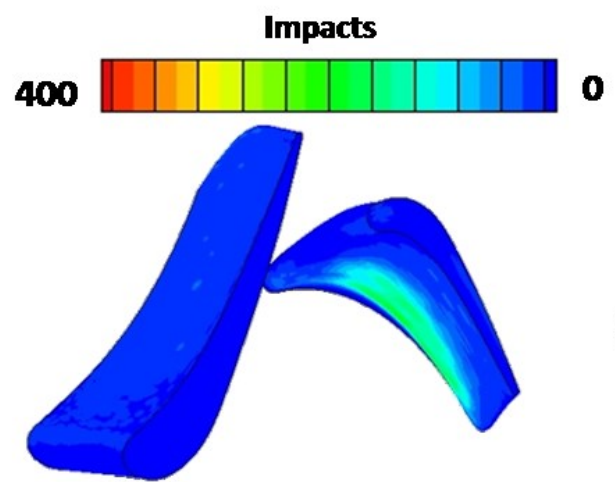

(a)
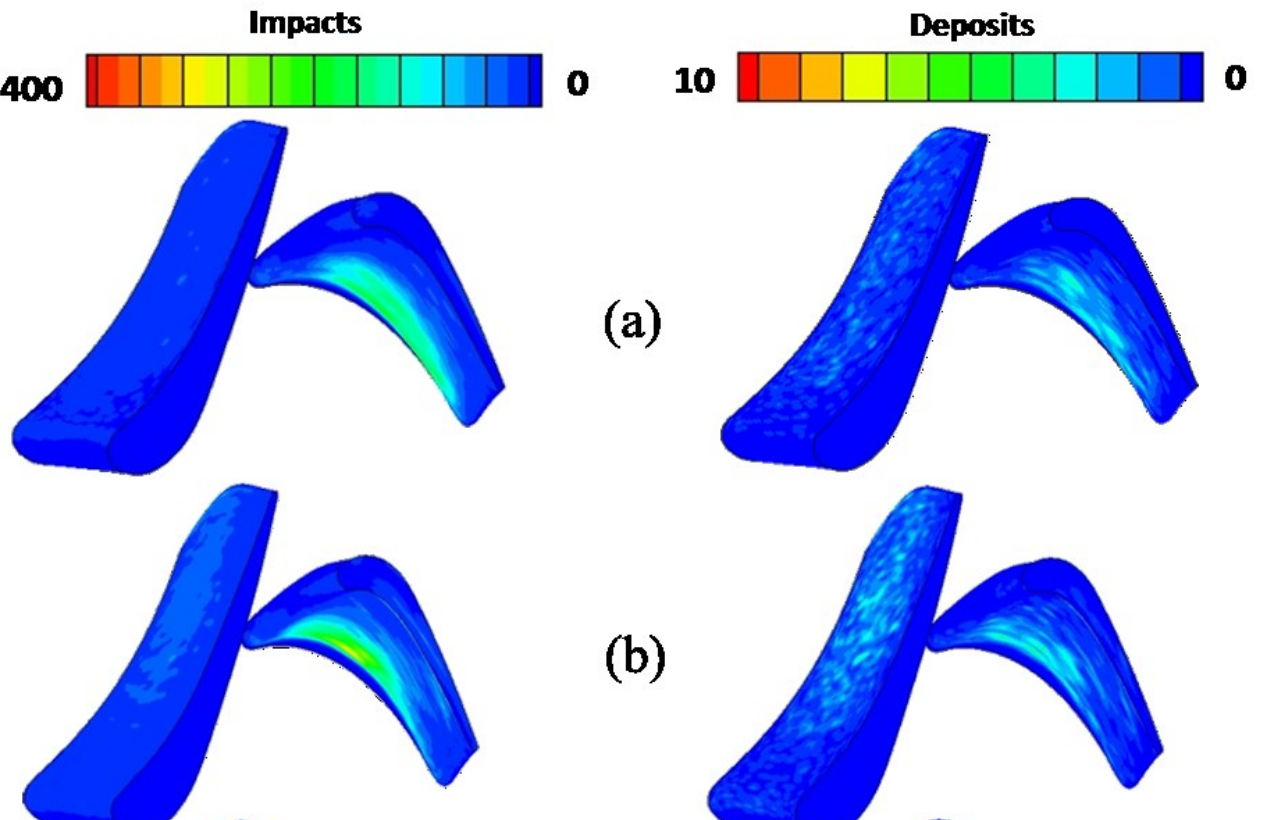

(b)
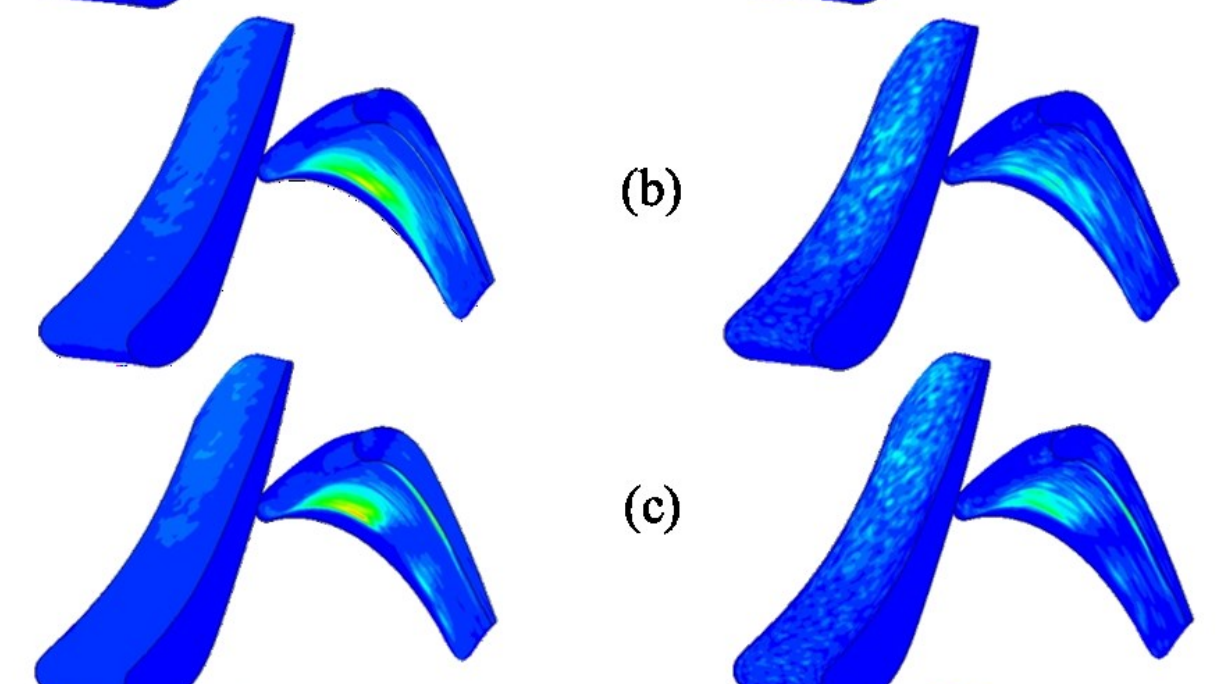

(c)
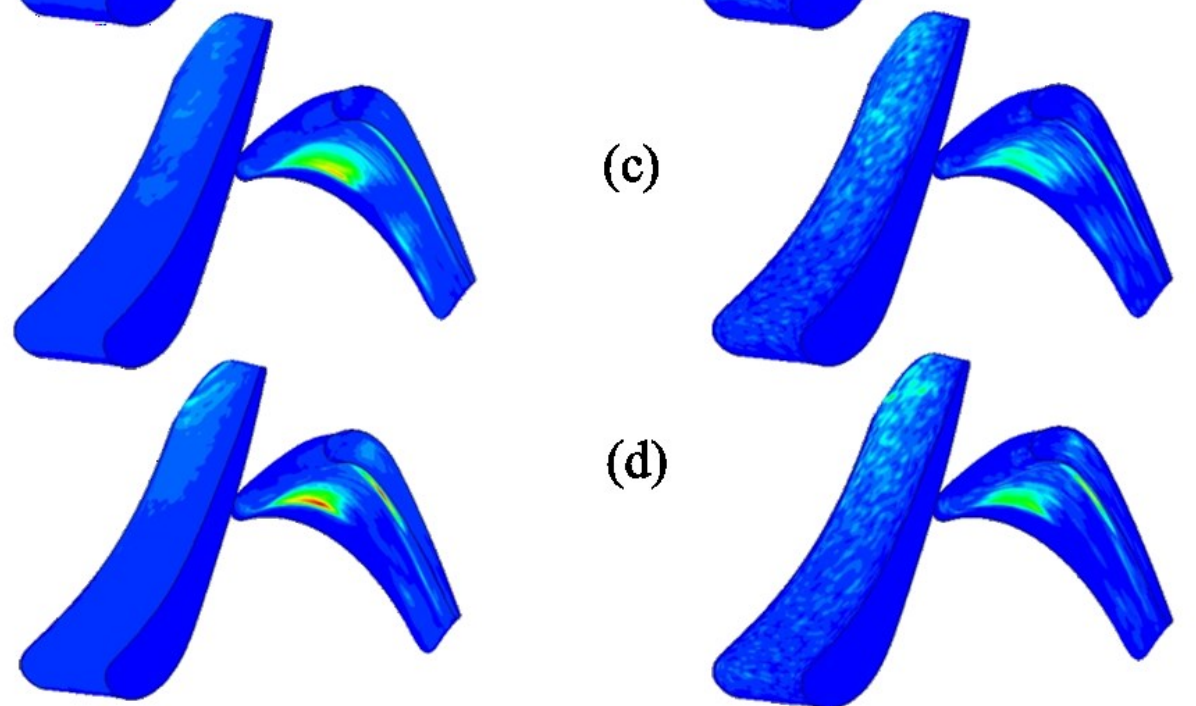

(d)

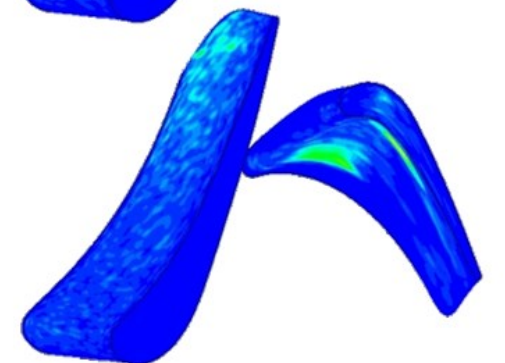

Figure E.1: Averaged impact and deposit contours for (a) $3 \mu \mathrm{m}$, (b) $6 \mu \mathrm{m}$, (c) $10 \mu \mathrm{m}$, (d) $15 \mu \mathrm{m}$ 

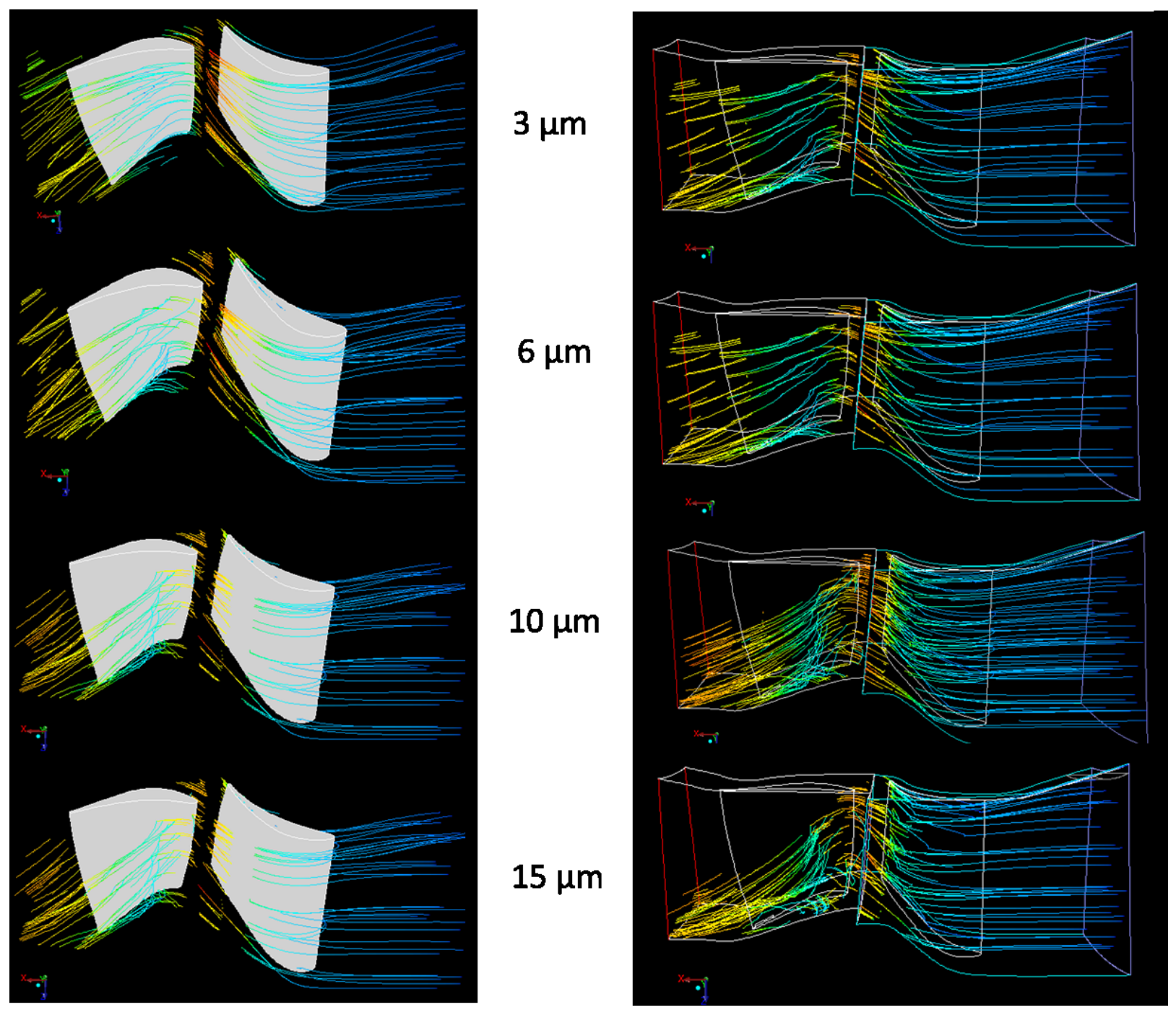

Figure E.2: Averaged method particle trajectories for different particle sizes 


\section{Appendix F - Particle Tracking Troubleshooting Chronology}

This list documents the steps that were performed in order to trouble shoot the unsteady particle tracking through the stage and to ultimately be able to run them in a parallel batch.

- FLUENT 13.0 unable to compile UDF in a serial or parallel batch

- 3 micron case run entirely in serial GUI due to this problem

- Summer Wang of OSC adds a few commands to UDF and recommends using new FLUENT 14.5 that includes auto compiler.

- Auto compiler works, UDF is incorporated.

- 10 micron case run in parallel batch FLUENT 14.5 .

- Particle trajectories begin to become nonphysical—huge velocities and backflow all the way to main inlet

- Develop small test mesh (two wedges with sliding interface)

- Test tracking particles through the domain in GUI vs. Serial Batch vs. Parallel Batch FLUENT 14.5.

No differences found. 
- Add an obstruction to test wedges and incorporate UDF for impact tracking and deposits. Test tracking particles through the domain in GUI vs. Serial Batch vs. Parallel Batch FLUENT 14.5.

- Still no differences found between any methods.

- Determined that flow solution may be becoming unstable in FLUENT 14.510 micron case. Residuals jump up a small amount at the time of particle trajectory divergence.

- Determine that it may be an issue with FLUENT 14.5 vs. FLUENT 13.0

- Develop a batch file using TUI commands that will compile the UDF in FLUENT 13.0 before running.

- Compiles successfully.

- 10 micron unsteady case run in FLUENT 13.0 successfully with a stable flow solution and stable particle tracking. 